\title{
Regressão não paramétrica com processos estacionários $\alpha$-mixing via ondaletas
}

\author{
Luz Marina Gómez Gómez
}

\author{
TESE APRESENTADA \\ $\mathrm{AO}$ \\ INSTITUTO DE MATEMÁTICA E ESTATÍSTICA \\ DA \\ UNIVERSIDADE DE SÃO PAULO \\ PARA \\ OBTENÇÃO DO TÍTULO \\ $\mathrm{DE}$ \\ DOUTOR EM CIÊNCIAS
}

\author{
Área de Concentração: Estatística \\ Orientador: Prof. Dr. Pedro Alberto Morettin
}

Durante o desenvolvimento deste trabalho o autor recebeu auxílio financeiro da CAPES e $\mathrm{CNPq}$

São Paulo, novembro de 2012 


\section{Regressão não paramétrica com processos estacionários $\alpha$-mixing, via Ondaletas}

Esta versão definitiva da tese contém as correções e alterações sugeridas pela Comissão Julgadora durante a defesa realizada por Luz Marina Gómez Gómez.

Banca Examinadora:

- Prof. Dr. Pedro Alberto Morettin (orientador) - IME-USP.

- Prof ${ }^{a}$ Dr $^{a}$ Chang Chiann- IME-USP.

- Prof $^{\mathrm{a}}$ Dr$^{\mathrm{a}}$ Silvia Regina Costa Lopes- UFRGS.

- Prof. Dr. João Ricardo Sato- UFABC

- Prof. Dr. Aluísio de Souza Pinheiro - UNICAMP 


\section{Agradecimentos}

- A Deus, por sempre guiar meu caminho, me dar coragem para superar os tropeços da vida e terme permitido concluir este trabalho.

- A meus pais, Nestor e Rosalba, a quen devo minha formação moral. Meu reconhecimento e gratidão pela paciência, compreensão e apoio constante, ainda na distância.

- A meu orientador, o professor Pedro Morettin, pela paciência e a excelente orientação.

- A meus irmãos pelo apoio e compreensão.

- A meus amigos e colegas do IME pela companhia; em especial Nubia, Deissy e Gustavo, pelo incentivo e colaboração para conseguir concluir este trabalho. A Michelle, pela ajuda com o português.

- A os professores do IME pela dedicação na árdua tarefa de ensinar.

- A CNPq e Capes pelo apoio financeiro.

- A banca examinadora pela presença e as valiosas sugestões 



\section{Resumo}

\section{Regressão não paramétrica com processos estacionários mixing via Ondaletas.}

Nesta tese consideramos um modelo de regressão não paramétrica, quando a variável explicativa é um processo estritamente estacionário e $\alpha$-mixing. São estudadas as condições sobre o processo $X_{t}$ e sua estrutura de dependência, assim como do domínio da função $f$ a ser estimada. Também são feitas as adaptações necessárias aos procedimentos para obter as taxas de convergência do risco para a norma $L_{p}$, no caso de ondaletas deformadas. Em relação às ondaletas adaptativas de Haar, obtém-se as taxas de convergência do risco do estimador proposto. Mediante estudos de simulação, é avaliado o desempenho dos procedimentos propostos quando aplicados a amostras finitas sob diferentes níveis de perturbação do sinal e diferentes tamanhos da amostra. Tambem são feitas aplicações a dados reais.

Palavras-chaves: regressão não paramétrica, ondaleta, processo estacionário, $\alpha$-mixing, ondaleta deformada, ondaleta adaptativa de Haar. 



\section{Abstract}

\section{Nonparametric regression with stationary mixing processes.}

In this thesis we consider a nonparametric regression model, when the exploratory variables are $\alpha$-mixing stationary processes. We obtain convergence rates for risk for $L_{p}$ norm, via warped wavelets, under suitable regularity conditions. For estimation using design adapted Haar wavelets we obtain convergence rates for the risk of the proposed estimator. The performance of the estimators are assessed via simulation studies with different sample sizes and different signal-to-noise ratios. Applications to real data are also given.

Key Words: nonparametric regression, wavelet, stationary process, $\alpha$-mixing, warped wavelet, adapted Haar wavelet. 



\section{Sumário}

Lista de Figuras $\quad$ ix

Lista de Tabelas $\quad$ xiii

1 Preliminares

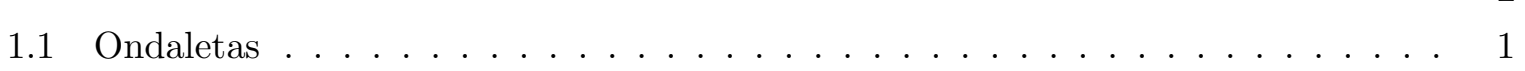

1.2 Espaços de funções . . . . . . . . . . . . . . . . . . . . 3

1.2.1 Espaços Ponderados de Besov . . . . . . . . . . . . . . . . . 6

1.3 Ondaletas Deformadas . . . . . . . . . . . . . . . . . . . 9

1.4 Ondaletas adaptativas de Haar . . . . . . . . . . . . . . . 11

1.5 Processos Mixing . . . . . . . . . . . . . . . . . . . . . . 14

1.6 Dependência Fraca . . . . . . . . . . . . . . . . . . . . . 15

1.7 Função de Hölder . . . . . . . . . . . . . . . . . . . . . . . . . . . 16

2 Modelo de Regressão com Variáveis

Fortemente Mixing Usando Ondaletas Deformadas $\quad 19$

2.1 Introdução . . . . . . . . . . . . . . . . . . . . . . . . . . . . . . . 19

2.2 Procedimento usando Ondaletas Deformadas . . . . . . . . . . . . . . 19

2.3 Resultados Principais . . . . . . . . . . . . . . . . . . . . 21

2.4 Simulações . . . . . . . . . . . . . . . . . . . . . . 25

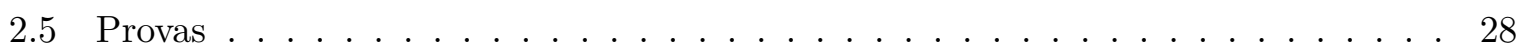

$2.5 .1 \quad$ Proposição $2.3 .1 \ldots \ldots \ldots \ldots \ldots \ldots \ldots$

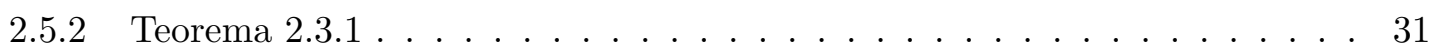

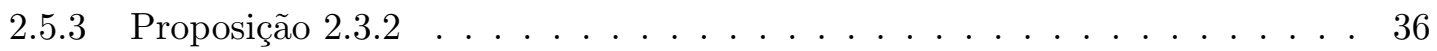

3 Usando Ondaletas Adaptativas $\quad 41$

3.1 Introdução . . . . . . . . . . . . . . . . . . . . . . 41

3.2 Procedimento usando Ondaletas Adaptativas de Haar . . . . . . . . . . . . . . 41

3.3 Resultados Principais . . . . . . . . . . . . . . . . . . . 44

3.4 Simulações . . . . . . . . . . . . . . . . . . . . . 47

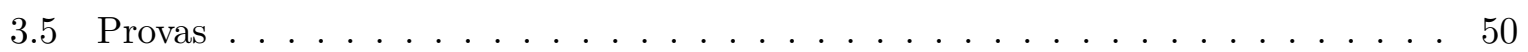

4 Aplicações $\quad 69$

4.1 Introdução . . . . . . . . . . . . . . . . . . . . . . . . . . . . 69

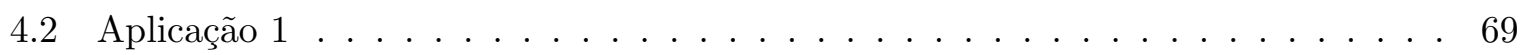


4.3 Aplicação 2, Modelo de Índice Único . . . . . . . . . . . . . . . . . . . . . . 75

4.3.1 Representação em Ondaletas do Modelo de Índice Único . . . . . . . . . . . 76

4.3.2 Aplicação a Dados de Poluição . . . . . . . . . . . . . . . . . . . . 78

5 Conclusões $\quad 83$

A Gráficos das simulações $\quad \mathbf{8 5}$

A.0.3 Graficos das Simulações com Ondaletas Deformadas . . . . . . . . . . . . 85

A.0.4 Graficos das Simulações com Ondaletas Adaptativas de Haar . . . . . . . 91

A.0.5 Graficos das Aplicações . . . . . . . . . . . . . . . . . . . . 92

$\begin{array}{ll}\text { Referências Bibliográficas } & 95\end{array}$ 


\section{Lista de Figuras}

2.1 Funções (a) $f(x)$ seno e $g(x)$ constante, (b) $f(x)$ seno e $g(x)$ seno, (c) $f(x)$ Heavisine e $g(x)$ constante, (d) $f(x)$ Heavisine e $g(x)$ seno, (e) $f(x)$ Doppler e $g(x)$ constante, (f) $f(x)$ Doppler e $g(x)$ seno; com $\mathrm{N}=2048$ e $\mathrm{rsn}=1$. As estimativas (linhas cheias) foram calculadas com a base symmlet 8. A função teórica é dada pelas linhas tracejadas. . . . . . . . . . . . . . . . . . . 27

3.1 Os pontos representam o planejamento aleatório do modelo 3.1. A linha tracejada representa a equação $Y_{t}=f\left(X_{t}\right)$, a verdadeira função. A linha contínua representa a função estimada com ondaletas adaptativas. As funções são: (a) função Seno com rsnr=7, (b) função f(x) Heavisine com rsnr=7, (c) Função Doppler com

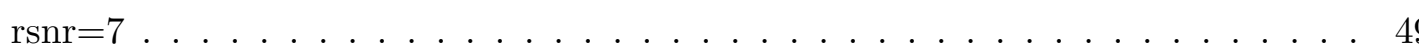

4.1 Gráfico de dispersão dos retornos mensais das ações ARCZ6 pelos retornos mensais do Índice Ibovespa . . . . . . . . . . . . . . . . . . . . . 70

4.2 Gráficos de: (a) retornos mensais do IBOVESPA, entre maio de 2001 e novembro de 2009, (b) retornos mensais do ARCZ6 entre maio de 2001 e novembro de 2009, (c) função de autocorrelação amostral dos retornos do IBOVESPA, (d) função de autocorrelação parcial amostral dos retornos do IBOVESPA (e) função de autocorrelação amostral dos retornos da ARCZ6, (f) função de autocorrelação parcial amostral dos retornos da ARCZ6 . . . . . . . . . . . . . . . . 71

4.3 Os pontos representam o gráfico de dispersão dos retornos das ações de ARCZ6 pelas flutuações do Ibovespa e a linha sólida representa a $\hat{\beta}$ obtida com ondaletas deformadas com base Symmlet6 e valor $\mathcal{K}=1,2 \ldots \ldots \ldots$. . . . . . . . 72

4.4 Gráficos dos resíduos obtido com ondaletas deformadas e base Symmlet6: (a) gráfico dos resíduos, (b)gráfico de quantis, (c)função de autocorrelação amostral, (d) função de autocorrelação parcial amostral. . . . . . . . . . . . . . . . . . 73

4.5 Gráfico de dispersão entre os retornos das ações ARCZ6 e retornos do Ibovespa:(a) a linha sólida representa a estimativa obtida com ondaletas adaptativas de Haar com todos os dados; (b) a linha sólida representa a estimativa obtida com ondaletas adaptativas de Haar tirando o máximo e o mínimo dos dados. . . . . . . 74

4.6 Gráficos dos resíduos obtidos com ondaletas adaptativas: (a) gráfico da série de resíduos, (b) gráfico de quantis, (c)função de autocorrelação, (d) função de autocorrelação parcial. . . . . . . . . . . . . . . . . . . 74

4.7 Gráfico de dispersão entre os retornos das ações ARCZ6 e retornos do IBOVESPA, a linha solida representa a estimativa obtida com ondaletas usuais. . . . . . . . . 75 
4.8 Gráficos dos resíduos obtido aplicando ondaletas usuais com base symlet6, (a)Gráfico dos resíduos, (b) quantil, (c)função de autocorrelação dos resíduos, (d) função de autocorrelação parcial dos resíduos. . . . . . . . . . . . . . 76

4.9 Funções: (a) gráfico da serie ozônio, (b)gráfico da série temperatura, (c) gráfico da série radiação, (d) gráfico da série velocidade do vento. . . . . . . . . . . . . . 79

4.10 (a) boxplot das estimativas de $\beta_{1}, \beta_{2}$ e $\beta_{3}$, respectivamente; (b) gráfico da série $X^{\prime} \hat{\beta}$ contra a série ozônio; (c) gráfico das curvas ajustadas para cada estimativa obtida do valor inicial dado; (d) apresenta a $\hat{f}$ estimada para o valor de $\hat{\beta}$. . . . 80

4.11 (a) Gráfico da função de autocorrelação amostral dos resíduos, (b) Gráfico QuantilQuantil dos resíduos. . . . . . . . . . . . . . . . . . . . 81

4.12 (a)boxplot das estimativas de $\beta_{1}, \beta_{2}$ e $\beta_{3}$, respectivamente; (b)gráfico da série $X \hat{\beta}$ contra a série ozônio; (c)gráfico das curvas ajustadas para cada estimativa obtida do valor inicial dado; (d)apresenta a $\hat{f}$ estimada para o valor de $\hat{\beta}$. . . . .

4.13 (a) Gráfico da função de autocorrelação amostral dos resíduos; (b) Gráfico QuantilQuantil dos resíduos. . . . . . . . . . . . . . . . . . . . .

A.1 Boxplot de MXDV para $f(x)=$ seno, $g(x)=$ constante, $(a),(b),(c)$ com $r s n=1$ e $N=512,1024$ e 2048, respectivamente, para $(d),(e),(f) r s n=7$ e $N=$ 512,1024 e 2048, respectivamente; V1 representa a base symmlet 8 e V2 representa a base coiflet $3 \ldots \ldots \ldots \ldots \ldots \ldots \ldots$

A.2 Boxplot de MXDV para $f(x)=$ seno, $g(x)=$ seno, $(a),(b),(c)$ com $r s n=1$ e $N=512,1024$ e 2048, respectivamente, para $(d),(e),(f) r s n=7$ e $N=512,1024$ e 2048, respectivamente; V1 representa a base symmlet 8 e V2 representa a base

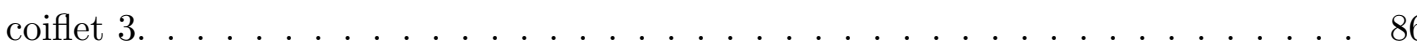

A.3 Boxplot de MXDV para $f(x)=$ Heavisine, $g(x)=$ constante e para $(a),(b),(c)$ $r s n=1$ e $N=512,1024$ e 2048, respectivamente, para $(d),(e),(f) r s n=7$ e $N=512,1024$ e 2048, respectivamente; V1 representa a base symmlet 8 e V2 representa a base coiflet $3 \ldots \ldots \ldots \ldots$. . . . . . . . . . . 87

A.4 Boxplot de MXDV para $f(x)=$ Heavisine, $g(x)=$ seno e para $(a),(b),(c) r s n=$ 1 e $N=512,1024$ e 2048, respectivamente, para $(d),(e),(f) r s n=7$ e $N=$ 512,1024 e 2048, respectivamente;V1 representa a base symmlet 8 e V2 representa a base coiflet 3 .

A.5 Boxplot de MXDV para $f(x)=$ doppler, $g(x)=$ constante e para $(a),(b),(c)$ $r s n=1$ e $N=512,1024$ e 2048, respectivamente, para $(d),(e),(f) r s n=7$ e $N=512,1024$ e 2048, respectivamente; V1 representa a base symmlet 8 e V2

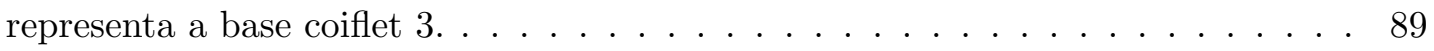

A.6 Boxplot de MXDV para $f(x)=$ doppler, $g(x)=$ constante e para $(a),(b),(c)$ $r s n=1$ e $N=512,1024$ e 2048, respectivamente, para $(d),(e),(f) r s n=7$ e $N=512,1024$ e 2048, respectivamente; V1 representa a base symmlet 8 e V2 representa a base coiflet $3 \ldots \ldots \ldots$. . . . . . . . . . . . . 90

A.7 Boxplot de MXDV para $\mathrm{n}=512, \mathrm{snr}=1$ e $f(x)$ :(a) Seno, (b) Heavisine (c) Doppler, com snr $=7$ e $f(x)$ (a) Seno, (b) Heavisine (c) Doppler, aplicando limiar para dados $\alpha$-mixing, e limiar para dados independentes. . . . . . . . . . . 
A.8 Boxplot de MXDV para $\mathrm{n}=1024$, snr=1 e $f(x)$ :(a) Seno, (b) Heavisine (c) Doppler, com snr=7 e $f(x)$ (a) Seno, (b) Heavisine (c) Doppler, aplicando limiar para dados $\alpha$-mixing, e limiar para dados independentes. . . . . . . . . . . . 92

A.9 Gráfico estimativas ondaleta deformada com $\mathcal{K}=0,5$ e base (a)Symmlet6, (b)Symmlet7, (c)Symmlet8, (d)Symmlet9, (e)Symmlet10, (f)Coiflet2, (g)Coiflet3, (h)Coiflet4, (i)Coiflet5. . . . . . . . . . . . . . . . . 93

A.10 Gráficos da função de autocorrelação dos resíduos das estimativas obtidas com ondaletas deformadas para $\mathcal{K}=0,5$ e base (a)Symmlet6, (b)Symmlet7, (c)Symmlet8, (d)Symmlet9, (e)Symmlet10, (f)Coiflet2, (g)Coiflet3, (h)Coiflet4, (i)Coiflet5. . . . 93

A.11 Gráfico estimativas ondaleta deformada com $\mathcal{K}=1,2$ e base (a)Symmlet6, (b)Symmlet7, (c)Symmlet8, (d)Symmlet9, (e)Symmlet10, (f)Coiflet2, (g)Coiflet3, (h)Coiflet4, (i)Coiflet5. . . . . . . . . . . . . . . . . . 94

A.12 Gráficos da função de autocorrelação dos resíduos das estimativas obtidas com ondaletas deformadas para $\mathcal{K}=1,2$ e base (a)Symmlet6, (b)Symmlet7, (c)Symmlet8, (d)Symmlet9, (e)Symmlet10, (f)Coiflet2, (g)Coiflet3, (h)Coiflet4, (i)Coiflet5. . . . 94 



\section{Lista de Tabelas}

2.1 RMSE do estudo de simulação. As colunas com "\%dif" são as diferenças percentuais entre o RMSE da base symmlet 8 e coiflet 3 . Foi atribuído o valor zero quando a diferença era insignificante com nivel 5\%, com o teste de Wilcox para amostras dependentes. . . . . . . . . . . . . . . . . . 26

3.1 RMSE do estudo de simulação. . . . . . . . . . . . . . . . . . . . . . 49 



\section{Introdução}

Regressão não paramétrica é uma técnica de recuperação de uma função de regressão a partir de dados observados com erro ou ruído, sem a necessidade de se especificar uma classe paramétrica de funções de regressão. Uma das técnicas usadas recentemente é a estimação via ondaletas. O uso de ondaletas em estatística foi iniciado por Donoho e Johnstone (1994), que apresentaram um conjunto de procedimentos coerentes, adaptativos e ótimos, em relação a uma ampla gama de classes de espaços de funções, com diversos graus de suavidade.

No caso de desenho aleatório, vários trabalhos foram apresentados como os de Hall e Turlach (1997), Antoniadis et al. (1997) e Antoniadis e Fan. (2001). Todos eles usaram um passo preliminar para voltar ao desenho equidistante e conseguir aplicar as tradicionais ondaletas ortogonais. Recentemente, surgiram mais duas abordagens para o problema do delineamento aleatório. Uma delas utiliza o conceito de ondaleta adaptativa, que propõe uma análise de ondaletas diretamente nos pontos do delineamento (Delouille (2002)). A outra estuda as condições sobre o delineamento em que os procedimentos de ondaletas continuam sendo adequados (Kerkyacharian e Picard (2004)). Para desenhos aleatórios na presença de dependência, pouco se conhece: Hafner (1998) estuda modelos ARCH com ondaletas que apresenta resultados assintóticos; Delouille e Sachs (2004) propõem um algoritmo usando ondaletas com desenho aleatório para estimar modelos autoregressivos.

Nesta tese consideramos um modelo de regressão não paramétrica, quando a variável explicativa é um processo estritamente estacionário e $\alpha$-mixing. São estudadas as condições sobre o processo $X_{t}$ e sua estrutura de dependência, assim como do domínio da função $f$ a ser estimada. Também são feitas as adaptações necessárias aos procedimentos para obter as taxas de convergência do risco para a norma $L_{p}$, no caso de ondaletas deformadas. Em relação às ondaletas adaptativas de Haar, obtém-se as taxas de convergência do risco do estimador proposto.

Mediante estudos de simulação, é avaliado o desempenho dos procedimentos propostos quando aplicados a amostras finitas sob diferentes níveis de perturbação do sinal e diferentes tamanhos da amostra. Também são feitas aplicações a dados reais.

A tese está organizada em 4 capítulos. No Capítulo 1 são apresentados alguns conceitos básicos sobre os quais se apóiam os procedimentos e demonstrações dos capítulos 2 e 3 . No Capítulo 2 são estudadas condições impostas à estrutura de dependência dos dados para que os procedimentos usuais de ondaleta possam ser usados. No Capítulo 3 são estudadas as taxas de convergência na presença de dados correlacionados e um limiar que leva em conta a estrutura 
de dependência. No Capítulo 4 são apresentadas duas aplicações que permitem mostrar as vantagens da metodologia proposta. 


\section{Capítulo 1}

\section{Preliminares}

Neste capítulo apresentamos algumas definições relacionadas com os espaços de funções, bem como conceitos e resultados relacionados com a metodologia de ondaletas deformadas e ondaletas adaptativas de Haar. A seguir, definiremos algumas classes de processos fracamente dependentes e alguns resultados sobre os quais são baseadas as provas dos resultados dados nos próximos capítulos.

\subsection{Ondaletas}

As ondaletas são funções localizadas no tempo (ou no espaço) e na escala, o que as torna apropriadas para analisar funções com descontinuidades (Morettin, 1999).

Uma base $\left\{\phi_{j, k}(t) \cup \psi_{j, k}(t), j, k \in \mathbb{Z}\right\}$, do espaço $L_{2}(\mathbb{R})$, pode ser gerada por meio de dilatações binárias e de traslações diádicas de uma ondaleta-pai $\phi$ (ou função escala) e de uma ondaleta-mãe $\psi$ :

$$
\phi_{j, k}(t)=2^{j / 2} \phi_{j, k}\left(2^{j} t-k\right), \quad \psi_{j, k}(t)=2^{j / 2} \psi_{j, k}\left(2^{j} t-k\right), j, k \in \mathbb{Z}
$$

A ortonormalidade destas funções depende da escolha das funções $\phi$ e $\psi$. Se $\psi$ (ou $\phi$ ) tiver suporte compacto em $[0, N]$, então $\psi_{j, k}$ (ou $\left.\phi\right)$ terá suporte em $\left[2^{-j} k, 2^{-j}(N+k)\right]$. A função $\phi$ deve satisfazer $\int \phi=1$ e $\int|\phi|<\infty$. A função $\psi$ deve ser tal que $\int \psi=0$ (admissível). Uma ondaleta é chamada r-regular se possuir r momentos nulos e r derivadas contínuas.

Uma base de ondaletas tem associada uma análise de multirresolução em $L_{2}(\mathbb{R})$ que per- 
mite que se analise os dados através de distintos níveis de resolução, adicionando ou desprezando detalhes. Mais formalmente, sejam $V_{j}$ e $W_{j}$ os subespaços fechados gerados por $\phi_{j, k}, k=0, \ldots, 2^{j}-1$ e $\psi_{j, k}, k=0, \ldots, 2^{j}-1$, respectivamente, então a análise associada de multirresolução satisfaz as seguintes condições:

1. $\ldots \subset V_{-1} \subset V_{0} \subset V_{1} \subset \ldots$;

2. $\overline{\cup_{j} V_{j}}=L_{2}(\mathbb{R})$;

3. $\cap_{j} V_{j}=\{0\}$;

4. $f(t) \in V_{j} \Longleftrightarrow f(2 t) \in V_{j+1}, \forall j$;

5. $V_{j+1}=V_{j} \oplus W_{j}, W_{j} \perp V_{j}$.

Assim, para um dado nível de resolução $j_{0}$, uma função $f \in L_{2}(\mathbb{R})$ pode ser expandida em uma série de ondaletas (no sentido de norma $L_{2}$ ):

$$
f(x)=\sum_{k=0}^{2^{j_{0}}-1} \alpha_{j_{0} k} \phi_{j_{0}, k}(x)+\sum_{j=j_{0}}^{\infty} \sum_{k=0}^{2^{j}-1} \beta_{j, k} \psi_{j, k}(x),
$$

em que

$$
\alpha_{j_{0}, k}=\left\langle f, \phi_{j_{0}, k}\right\rangle, \quad \beta_{j, k}=\left\langle f, \psi_{j, k}\right\rangle
$$

Esta transformação de ondaleta decompõe uma função em componentes com diferentes resoluções. Os coeficientes do nível mais grosso são os $\alpha_{j_{0}, k}$, representando a estrutura suave da função $f$. Os coeficientes de detalhes são denotados por $\beta_{j, k}$ e representam as estruturas cada vez mais finas em $f$, à medida em que o nível de resolução $j$ aumenta. Diferentes ondaletas caracterizam diferentes espaços tradicionais de funções como os de Hölder, Sobolev e Besov.

Em aplicações são amostrados dados da função $f$ e há uma base ortonormal de ondaletas, que está geralmente associada a uma transformada discreta de ondaletas. Esta transforma os dados amostrados para o domínio dos coeficientes de ondaletas. Nos métodos usuais que utilizam ondaletas, os dados são observações das variáveis aleatórias $Y_{1}, \ldots, Y_{N}$, onde $Y_{i}=f(i / N)+e_{i}$, a função $f$ é desconhecida e os erros (ou ruídos) $e_{i}$ possuem média zero e variância $\sigma^{2}<\infty$. 
Usando uma transformada discreta, os dados são transformados em coeficientes (empíricos) de ondaletas e um estimador da função $f$ é obtido por meio da transformada inversa dos coeficientes, após a redução ou remoção do ruído. A função $f$ é desconhecida e há interesse em estimá-la globalmente com o menor error quadrático meio IMSE

$$
R_{2}(\hat{f}, f)=E\|\hat{f}-f\|_{2}^{2}=\int_{0}^{1} E(\hat{f}(x)-f(x))^{2} d x
$$

em que o estimador $\hat{f}$ é dado de forma genérica por:

$$
\hat{f}=\sum_{k=0}^{2^{j_{0}}-1} \hat{\alpha}_{j_{0}, k} \phi_{j_{0}, k}(x)+\sum_{j=j_{0}}^{J} \sum_{k=0}^{2^{j}-1} \hat{\beta}_{j, k} \psi_{j, k}(x)
$$

seguindo a equação (1.2). Estimativas dos coeficientes são geralmente obtidas aplicando-se limiares aos coeficientes empíricos que, por sua vez, são obtidos por diferentes métodos, alguns dos quais são descritos nas seções seguintes.

\subsection{Espaços de funções}

Nesta seção são apresentados resultados de aproximação em espaços de Besov. Estes são mais gerais que os espaços de Sobolev para descrever as propriedades de suavidade das funções. Os resultados apresentados a seguir podem ser encontrados em Hardle et al. (1998).

Definição 1.2.1 Seja $f$ uma função em $L_{p}(R), 1 \leq p<+\infty$, e sejam $\tau_{h}(f(x))=f(x-h)$, $\Delta_{h} f(x)=\tau_{h}(f(x))-f(x)$. Definimos também $\Delta_{h}^{2} f(x)=\Delta_{h} \Delta_{h}(f(x))$ e assim para $\Delta_{h}^{n} f(x)$, $n \in \mathbb{N}^{*}$. Para todo $t \geq 0$ os módulos de continuidade são definidos como:

$$
\omega_{p}^{1}(f, t)=\sup _{|h| \leq t}\left|\Delta_{h} f(x)\right|, \quad \omega_{p}^{2}(f, t)=\sup _{|h| \leq t}\left|\Delta_{h}^{2} f(x)\right|
$$

Para uma sequência $a=\left\{a_{j}\right\}, j=1,2, \ldots$, de números reais, e $1 \leq p<+\infty$, considere a norma

$$
\|a\|_{l_{p}}= \begin{cases}\left(\sum_{j=0}^{+\infty}\left|a_{j}\right|^{p}\right)^{\frac{1}{p}}, & 1 \leq p<+\infty \\ \sup _{j}\left|a_{j}\right|, & p=\infty,\end{cases}
$$


em que $l_{p}$ denota o espaço de todas as sequências $a=\left\{a_{j}\right\}$ tais que $\|a\|_{l_{p}}<+\infty$. Seja $1 \leq q \leq+\infty$ dado, e seja a função $\epsilon_{t}$ em $[0,+\infty]$ tal que $\|\epsilon\|_{q}^{*}<+\infty$, em que

$$
\|\epsilon\|_{q}^{*}= \begin{cases}\left(\int_{0}^{\infty}|\epsilon(t)|^{q} \frac{d t}{t}\right)^{\frac{1}{q}}, & 1 \leq q<+\infty \\ \operatorname{ess~sup}_{t}\left|\epsilon_{t}\right|, & q=\infty\end{cases}
$$

Definição 1.2.2 Seja $1 \leq p<+\infty, m \in\{1,2, \ldots\}$. A função $f \in L_{p}(\mathbb{R})$ pertence ao espaço de Sobolev $W_{p}^{m}(\mathbb{R})$, se esta é $m$ vezes diferenciável e se $f^{(i)} \in L_{p}(\mathbb{R})$ para $i=1,2, \ldots, m$. Os espaços de Sobolev tem a norma associada $\|f\|_{p}+\left\|f^{(m)}\right\|_{p}$.

Definição 1.2.3 O espaço $\tilde{W}_{p}^{m}(\mathbb{R})$ é definido como segue:

$$
\tilde{W}_{p}^{m}(\mathbb{R})= \begin{cases}W_{p}^{m}(\mathbb{R}), & \text { se } 1 \leq p<\infty \\ \left\{f \in W_{\infty}^{m}(\mathbb{R}): f^{(m)} \text { é uniformemente continua }\right\}, & \text { se } p=\infty,\end{cases}
$$

em particular $\tilde{W}_{p}^{0}(\mathbb{R})=L_{p}(\mathbb{R})$, para $1 \leq p<+\infty$.

Definição 1.2.4 Seja $1 \leq p, q<\infty$ e $s=n+\alpha$, com $n \in\{0,1, \ldots\}$ e $0<\alpha \leq 1$. O espaço de Besov $B_{p}^{s p}(\mathbb{R})$ é o espaço de todas as funções $f$ tal que $f \in W_{p}^{n}(\mathbb{R}), \omega_{p}^{2}\left(f^{(n)}, t\right)=\epsilon(t) t^{\alpha}$, em que $\|\epsilon\|_{q}^{*}<\infty$. A norma do espaço $B_{p}^{s p}(\mathbb{R})$ é definida por:

$$
\|f\|_{s p q}=\|f\|_{W_{p}^{n}}+\left\|\frac{\omega_{p}^{2}\left(f^{(n)}, t\right)}{t^{\alpha}}\right\|_{q}^{*}
$$

Uma definição equivalente é:

Definição 1.2.5 O espaço de Besov $\left(B_{p}^{s q}\right)(\mathbb{R})$ é o espaço de todas as funções $f$ tal que $f \in$ $W_{p}^{n}(\mathbb{R})$ e $\left\{2^{j \alpha} \omega_{p}^{2}\left(f^{(n)}, 2^{-j}\right), j \in \mathbb{Z}\right\} \in l_{q}(\mathbb{Z})$. A norma é dada por:

$$
\|f\|_{s p q}=\|f\|_{W_{p}^{n}}+\left\|\left\{2^{j \alpha} \omega_{p}^{2}\left(f^{(n)}, 2^{-j}\right)\right\}\right\|_{l_{q}(\mathbb{Z})} .
$$

Definição 1.2.6 Um sistema de funções $\left\{\rho_{k}, k \in \mathbb{Z}\right\}, \rho_{k} \in L_{2}(\mathbb{Z})$, é chamado sistema ortonormal (ONS) se $\int \rho_{k}(x) \rho_{j}(x) d x=\delta_{j k}$, em que $\delta_{j k}$ é o delta de kronecker.

Vamos estabelecer as seguintes suposições. 
(C1) Seja $\{\rho(x-k), k \in \mathbb{Z}\}$ um sistema ortonormal que pode ser expresso pela relação

$$
\sum_{k}|\hat{\rho}(\xi+2 \pi)|^{2}=1 \text { q.c. }
$$

em que $\hat{\rho}$ representa a transformada de Fourier de $\rho$.

(C2) Pelo resultado 5.1 de Hardle et al. (1998), o espaço $V_{j}$ é encaixado se e somente se $\hat{\rho}(\xi)=$ $\hat{\rho}\left(\frac{\xi}{2}\right) m_{0}\left(\frac{\xi}{2}\right)$, q.c. em que $m_{0}(\xi)$ é uma função $2 \pi$-periódica, com $m_{0}(\xi) \in L_{2}(0,2 \pi)$. Os $V_{j}$ são subespaços fechados, gerados por $\phi_{j, k}$ na análise multirresolução.

(C3) A função $\theta_{\rho(x)}=\sum_{k}|\rho(x-k)|$ é tal que ess $\sup _{x} \theta_{\rho(x)}<\infty($ condição $\theta)$.

(C4) Existe uma função limitada e não crescente $\Phi$ tal que $\int \Phi(|u|) d u<\infty$ e $|\rho(u)| \leq \Phi(|u|)$ (condição S).

(C5) A condição (C4) satisfeita e $\int \Phi(|u|)|u|^{N} d u<\infty$ (condição $\mathrm{S}(\mathrm{N})$ ).

Observação:

Note que se $\rho$ satisfaz a suposição (C3), então $\rho \in L_{\infty}(\mathbb{R})$, e também $\theta_{\rho}$ é uma função periódica com período 1 e com $\int_{0}^{1} \theta(x)_{\rho(x)} d x<\infty$. Além disso, $\int_{0}^{1} \sum_{k}|\rho(x-k)| d x=$ $\int_{0}^{1} \theta_{\rho}(x) d x<\infty$.

Definição 1.2.7 O kernel $K(x ; y)$ é uma função definida em $\mathbb{R} \times \mathbb{R}$. Se $K(x, y)=K(x-y)$, então K é chamado um kernel de convolução.

Seja K $(x, y)$ um kernel. Para um número real positivo $h$, define-se $K_{h}(x, y)=h^{-1} K\left(h^{-1} x, h^{-1} y\right)$. Se $h=2^{-j}$, escrevemos $K_{j}(x, y)$ em vez de $K_{h}(x, y)$.

Para uma função $f$ mensurável apresentamos o operador associado com o kernel: $K_{h} f(x)=$ $\int K_{h}(x, y) f(y) d y$. Analogamente são definidas $K_{j} f$ e $K f$.

Teorema 1.2.1 Seja o Kernel $K$ satisfazendo a condição $M(N)$, e condição $H(N+1)$, para algum enteiro $N \geq 0$. Seja $1 \leq p, q \leq \infty$ e $0<s<N+1$. Se $f \in B_{p}^{s q}(\mathbb{R})$, então:

$$
\left\|K_{j} f-f\right\|_{p}=2^{-j s} \epsilon_{j}
$$

em que $\left\{\epsilon_{j}\right\} \in l_{q}$. 
Teorema 1.2.2 Seja $\rho$ a função escala satisfazendo as suposições $(C 1)-(C 3)$. Seja $N \geq 0$ um inteiro. Suponha que $\rho$ é $N+1$ vezes diferenciável e que a derivada $\rho^{(N+1)}$ satisfaz a condição (C3). Então, para todo $0<s<N+1,1 \leq p, q \leq \infty$, para qualquer função $f \in L_{p}(\mathbb{R})$ temos:

i) $\left\|K_{j} f-f\right\|_{p}=\epsilon_{j} 2^{-j s}, j=0,1, \ldots \operatorname{com} \epsilon_{j} \in l_{q} \Rightarrow f \in B_{p}^{s q}(\mathbb{R})$,

ii) $\left\|\alpha_{0}\right\|_{l_{p}}<\infty e\left\|\beta_{j}\right\|_{l_{p}}=2^{-j\left(s+\frac{1}{2}-\frac{1}{p}\right)} \epsilon_{j}^{\prime}, j=0,1,2, \ldots, \operatorname{com}\{\epsilon\}_{j}^{\prime} \in l_{q} \Rightarrow f \in B_{p}^{s q}(\mathbb{R})$.

Teorema 1.2.3 Seja $\rho$ uma função escala satisfazendo $(C 1)-(C 2)$ e $(C 5)$ (condição $S(N))$ com $N+1$ em vez de $N$, para algum $N \geq 0$. Suponha que $\rho$ é $N+1$ vezes diferenciável e que a derivada $\rho^{(N+1)}$ satisfaz a condição $\theta$. Então para algum $0<s<N+1,1 \leq p, q \leq \infty$, para toda $f \in L_{p}(\mathbb{R})$ as seguintes condições são equivalentes:

B1) $f \in B_{p}^{s q}(\mathbb{R})$;

B2) $\left\|K_{j} f-f\right\|_{p}=\epsilon_{j} 2^{-j s}, j=0,1, \ldots \operatorname{com}\left\{\epsilon_{j}\right\} \in l_{q}$;

B3) $\left\|\alpha_{0}\right\|_{l_{p}}<\infty e\left\|\beta_{j}\right\|_{l_{p}}=2^{-j\left(s+\frac{1}{2}-\frac{1}{p}\right)} \epsilon_{t}^{\prime}, j=0,1, \ldots$, onde $\left\{\epsilon_{t}^{\prime}\right\} \in l_{q}$.

Corolário 1.2.1 Sob as suposições do teorema 1.2.3, a norma de Besov $\|f\|_{s p q}, 1 \leq q \leq \infty$ é equivalente a seguinte norma no espaço de coeficientes de ondaleta:

$$
\|f\|_{s p q}^{\prime}=\left(\sum_{k}\left|\alpha_{k}\right|^{p}\right)^{\frac{1}{p}}+\left[\sum_{j=0}^{\infty}\left(2^{j\left(s+\frac{1}{2}-\frac{1}{p}\right)}\left(\sum_{k}\left|\beta_{j k}\right|^{p}\right)^{\frac{1}{p}}\right)^{q}\right]^{\frac{1}{q}}
$$

\subsubsection{Espaços Ponderados de Besov}

As definições e resultados apresentados nesta seção podem ser estudados detalhadamente em Kerkyacharian e Picard (2004).

Seja:

$$
\rho^{N}(t, f, \omega, p)=\sup _{|h| \leq t}\left(\int\left|\Delta_{h}^{N} f(u)\right|^{p} \omega(u) d u\right)^{\frac{1}{p}}
$$

com a modificação usual para $p=\infty$, definimos o seguinte espaço de Besov modificado:

$$
B_{s, p, q}(\omega)=\left\{f:\left(\int_{0}^{1}\left(\frac{\rho^{N}(t, f, \omega, p)}{t^{s}}\right)^{q} \frac{d t}{t}\right)^{\frac{1}{q}}<\infty\right\} .
$$


A diferença com o espaço de Besov usual é o fato de que o módulo de continuidade $\rho^{N}$ é calculado sobre um espaço ponderado pela medida $\omega(u) d u$ em vez da medida de Lébesgue, correspondente à função $\omega=1$.

Uma das grandes vantagens dos espaços regulares de Besov é que eles podem ser expressos em termos de coeficientes de ondaletas, sob condições padrões de oscilação da ondaleta $\psi$. Temos para $f=\sum_{j \geq-1, k} \beta_{j k} \psi_{j k}$,

$$
\left(\int_{0}^{1}\left(\frac{\rho^{N}(t, f, \omega, p)}{t^{s}}\right)^{q} \frac{d t}{t}\right)^{\frac{1}{q}}<\infty \Leftrightarrow\left[\sum_{j \geq-1}\left\{2^{j s} 2^{\frac{j}{2}}\left(\sum_{k \in \mathbb{Z}}\left|\beta_{j, k}\right|^{p} 2^{-j}\right)^{\frac{1}{p}}\right\}^{q}\right]^{\frac{1}{q}}<\infty
$$

A continuação são apresentadas duas definições necessárias para enunciar algumas proposições relacionadas com os espaços de Besov ponderados.

Definição 1.2.8 Se B é o conjunto de todos os intervalos de $\mathbb{R}$ e se $\omega$ é uma função mensurável, então a função maximal de Hardy-Littlewood associada a $\omega$ é

$$
\omega^{*}=\sup _{I \in B, x \in I}\left(\frac{1}{|I|} \int_{I} \omega(u) d u\right)
$$

Definição 1.2.9 Para $1<p<\infty, 1 / p+1 / q=1$, uma função mensurável $\omega \geq 0$ é um peso de Muckenhoupt (pertence à classe $A_{p}$ de Muckenhoupt), se existe $0<C<\infty$ tal que para qualquer intervalo $I \subset \mathbb{R}$,

$$
\left(\frac{1}{|I|} \int_{I} \omega(u) d u\right)^{1 / p}\left(\frac{1}{|I|} \int_{I} \omega(u)^{-q / p} d u\right)^{1 / q} \leq C
$$

para $p=1, \omega \geq 0$ pertence a classe $A_{1}$ de Muckenhoupt se existe $0<C<\infty$ tal que

$$
\omega^{*} \leq C \omega(x) \quad \text { q.c. }
$$

em que $\omega^{*}$ é uma função maximal de Hardy-Littlewood. Para $p=\infty$, definimos

$$
A_{\infty}=\cup_{A \geq 1} A_{p}
$$

Os espaços de Muckenhoupt são crescentes em p e englobam diversas funções: por exemplo, 
uma função limitada superior e inferior, pertence a $A_{p}$, para todo $p \geq 1$. A definição de peso de Muckenhoupt de certa forma quantifica quão próximo $\omega$ está de um peso uniforme, em que a função e sua inversa ponderam igualmente cada intervalo.

Proposição 1.2.1 Para $1 \leq p \leq \infty$, suponhamos que $\omega$ está em $A_{p}$, a classe de pesos de Muckenhoupt, vamos definir, para cada intervalo $I \subset \mathbb{R}$,

$$
\omega(I)=\int_{I} \omega(x) d x
$$

Então, se $\psi$ é uma ondaleta de suporte compacto tal que

$$
\int \psi(x) x^{k} d x=0 \text { para } k=0, \ldots, N-1,
$$

então para $f=\sum_{j, k} \beta_{j k} \psi_{j k}$ e $I_{j k}=\left[\frac{k}{2^{j}}, \frac{k+1}{2^{j}}\right]$

$$
\left(\int_{0}^{1}\left(\frac{\rho^{N}(t, f, \omega, p)}{t^{s}}\right)^{q} \frac{d t}{t}\right)^{1 / q}<\infty \Rightarrow\left[\sum_{j}\left\{2^{j s} 2^{\frac{j}{2}}\left(\sum_{k \in \mathbb{Z}}\left|\beta_{j k}\right|^{p} \omega\left(I_{j k}\right)\right)^{1 / p}\right\}^{q}\right]^{1 / q}<\infty,(1
$$

com as modificações usuais para $q=\infty$.

Seja $G$ a função de distribuição do planejamento, define-se $\Delta_{h}(G) f(x)=f\left(G^{-1}[G(x)+h]\right)$ $f(x)$, de forma recursiva $\Delta_{h}^{2}(G) f(x)=\Delta_{h}(G)\left(\Delta_{h}(G) f\right)(x)$ e do mesmo modo $\Delta_{h}^{N}(G) f(x)$, $N \in \mathbb{N}^{*}, \mathrm{e}$

$$
\tilde{\rho}^{N}(t, f, G, p)=\sup _{|h| \leq t}\left(\int\left|\Delta_{h}^{N}(G) f(u)\right|^{p} d u\right)^{\frac{1}{p}} .
$$

Deste modo, é definido o espaço:

$$
B_{s, p, q}^{G}=\left\{f:\left(\int_{0}^{1}\left(\frac{\tilde{\rho}^{N}(t, f, G, p)}{t^{s}}\right)^{q} \frac{d t}{t}\right)^{\frac{1}{q}}<\infty\right\}
$$

Corolário 1.2.2 Sob as condições da Proposição 1.2.1 e da condição (1.27)(dada na próxima 
seção) para $f=\sum_{j \geq-1, k} \beta_{j k} \psi_{j k},\left(\right.$ ou seja $\left.\beta_{j k}=\int\left[f \circ G^{-1}\right] \psi_{j k}\right)$, temos

$$
\left(\int_{0}^{1}\left(\frac{\tilde{\rho}^{N}(t, f, G, p)}{t^{s}}\right)^{q} \frac{d t}{t}\right)^{\frac{1}{q}}<\infty \Rightarrow\left[\sum_{j}\left\{2^{j s} 2^{j / 2}\left(\sum_{k \in \mathbb{Z}}\left|\beta_{j k}\right|^{p} \omega\left(I_{j, k}\right)\right)^{1 / p}\right\}^{q}\right]^{1 / q}<\infty
$$

com as modificações usuais para $q=\infty$.

\subsection{Ondaletas Deformadas}

Os resultados apresentados nesta seção podem ser consultados em Kerkyacharian e Picard (2004) e Porto (2008).

Considere o modelo

$$
Y_{i}=f\left(X_{i}\right)+\epsilon_{i}
$$

para $i=1,2, \ldots, N, N=2^{J}, J \in \mathbb{N}$, em que $X_{i}$ são variáveis aleatórias i.i.d com distribuição $G$ conhecida e $g$ a respectiva densidade, com suporte compacto em $[a, b]$. Além disso, suponha que $\epsilon_{i}$ sejam variáveis $i . i . d$ independentes de $X_{i}$, seguindo alguma densidade com média zero e variância $\sigma^{2}$ conhecida.

Sejam $a \leq X_{(1)}<X_{(2)}<\ldots<X_{(N)} \leq b$ as estatísticas de ordem dos $X_{i}$. Substituindo os rótulos de acordo com a ordem dos $X_{i}^{\prime} s$, o modelo pode ser escrito como:

$$
Y_{i^{*}}=f\left(X_{(i)}\right)+e_{i} \quad i=1,2, \ldots, N
$$

em que $Y_{i^{*}} \equiv Y\left(X_{(i)}\right)$ e $e_{i}=\epsilon_{i} \Leftrightarrow X_{(i)}=X_{j}, j=1,2, \ldots, N$. Os dados consistem de pares de variáveis aleatórias $\left(X_{(1)}, Y_{1^{*}}\right),\left(X_{(2)}, Y_{2^{*}}\right), \ldots,\left(X_{(N)}, Y_{N^{*}}\right)$. Se $X_{(i)}$ for uma boa aproximação para $G^{-1}(i / N)$, então o modelo (1.25) pode ser aproximado por:

$$
Y_{i^{*}}=f\left(G^{-1}(i / N)\right)+e_{i} \quad i=1,2, \ldots, N
$$

Expandindo a função $f\left(G^{-1}(i / N)\right) \equiv f \circ G^{-1}(y)$ em uma base ortonormal de ondaletas $\left\{\phi_{j_{0}, k}(t) \cup \psi_{j, k}(t), j \geq j_{0}, k \in \mathbb{Z}\right\}$, os coeficientes aproximam, respectivamente, aqueles da expansão da função $f$, em uma base de ondaleta deformada por $\mathrm{G}$, como mostrado em Porto (2008). 
A nova base formada por ondaletas deformadas não é mais ortonormal mas é computacionalmente simples, permitindo que se utilizem métodos já desenvolvidos. Nesse sentido, a especificação das condições necessárias à distribuição $G$, para que essa aproximação seja satisfatória, generalizam os resultados de Cai and Brown (1999).

De acordo com Kerkyacharian e Picard (2004), a seguinte condição é suficiente para que a expansão da função f, em uma base de ondaletas deformadas por G, seja bem aproximada pela expansão de $f \circ G^{-1}$, na base de ondaletas não deformadas correspondente:

$$
y \longmapsto \omega(y)=\frac{1}{g\left(G^{-1}(y)\right)} \in A_{p}([a, b]) .
$$

Isso de certa forma quantifica a suposição usual de que o delineamento atribua densidade suficiente a qualquer intervalo do suporte da função f. Tipicamente, se $G$ for definido em $(a, b)$ e se $G^{-1}$ for localmente Lipschitz em $(0,1)$, então, $\omega \in A_{p}$ com $1<p<\infty$ e

$$
G^{-1}(G(x))=x \quad \text { q.c., } \quad G\left(G^{-1}(y)\right)=y \quad \text { q.c., } \quad \text { e } \int_{\mathbb{R}} h(G(x)) d x=\int_{\mathbb{R}} h(y) \omega(y) d y,
$$

para qualquer função mensurável $h$ não negativa (Kerkyacharian e Picard (2004)).

Para muitas aplicações estatísticas, especialmente do ponto de vista assintótico, uma base de ondaletas deformadas $\phi_{j_{0}, k}(G(t)) \cup \psi_{j, k}(G(t)), j \geq j_{0}, k \in \mathbb{Z}$ gera uma aproximação satisfatória se satisfaz as seguintes propriedades:

Definição 1.3.1 Uma base $\left\{\zeta_{i}, i \in \mathbb{N}\right\}$ possui a propriedade de encolhimento (ou incondicional) se existe uma constante $C>0$ tal que

$$
\left\|\sum_{i \in \mathbb{N}} d_{i} \zeta_{i}\right\|_{p} \leq C\left\|\sum_{i \in \mathbb{N}} d_{i}^{\prime} \zeta_{i}\right\|_{p}
$$

se $\left|d_{i}\right| \leq\left|d_{i}^{\prime}\right|$.

Definição 1.3.2 Uma base $\left\{\zeta_{i}, i \in \mathbb{N}\right\}$ possui a propriedade $p$-Temlyakov se existem constantes $c_{p}$ e $C_{p}$ tais que

$$
c_{p} \int \sum_{i \in F}\left|\zeta_{i}\right|^{p} \leq \int\left(\sum_{i \in F}\left|\zeta_{i}\right|^{2}\right)^{p / 2} \leq C_{p} \int \sum_{i \in F}\left|\zeta_{i}\right|^{p},
$$

para qualquer conjunto finito $F$ de inteiros. Em uma base ortogonal $c_{p}=C_{p}$. 
Essas propriedades são verdadeiras para $p=2$ quando $\zeta_{i}$ é uma base ortonormal, permitindo uma extensão para $p>2$. Também são verdadeiras para bases de ondaletas com suporte compacto, mas em geral são falsas para bases deformadas, mesmo para $p=2$. Sob certas condições, vale o seguinte teorema de Kerkyacharian e Picard (2004).

Teorema 1.3.1 Sob a condição (1.28), a base de ondaletas deformadas $\left\{\phi_{j_{0}, k}(G().) \cup \psi_{j, k}(G()),. j \geq j_{0}, k \in \mathbb{N}\right\}$ satisfaz as condições de encolhimento e p-Temlyakov.

Assim como as ondaletas no caso igualmente espaçado, as ondaletas deformadas possuem também as seguintes propriedades:

1) $E\left[\psi_{j, k}(G(x))\right]=\int_{0}^{1} \psi_{j, k}(y) d y=0$;

2) $E\left[\psi_{j, k}^{2}(G(x))\right]=\int_{0}^{1} \psi_{j, k}^{2}(y) d y=1$.

Como geralmente na prática a distribuição $G$ não é conhecida, usa-se a distribuição empírica e isto é equivalente a se realizar uma estimação usando ondaletas pelo procedimento VisuShrink, com limiar duro, tratando os dados como se fossem igualmente espaçados. Pode-se mostrar que, quando o delineamento satisfaz à condição (1.27), o estimador $\hat{f}$ obtido é quase minimax-ótimo, sobre uma ampla classe de funções (Kerkyacharian e Picard (2004)).

\subsection{Ondaletas adaptativas de Haar}

Seja $L_{2}(d G) \equiv L_{2}(I, \Sigma, \mu)$ um espaço ponderado, onde $I \subseteq \mathbb{R}, \Sigma=\sigma(I)$ e $\mu=d G$ é uma medida de Borel-Stieltjes $\sigma$-finita em $\Sigma$ associada a G. Seja $G^{-1}(u)=\inf \{x: G(x) \geq u\}$ e denote por $\hat{G}$ a distribuição empírica de $G$ com uma medida discreta $\hat{\mu}=d \hat{G}$ associada. A abordagem de ondaletas adaptativas consiste em decompor uma função $f(x)$ em uma base de ondaletas $\left\{\phi_{j_{0}, k}(t) \cup \psi_{j, k}(t), j=j_{0}, \ldots, J, j_{0} \in 1,2, \ldots, J, k \in \mathcal{K}_{j}\right\}$ que é ortogonal no espaço $L_{2}(d G):$

$$
f(x)=\sum_{k \in \mathcal{K}_{j}} s_{j_{0}, k} \phi_{j_{0}, k}(x)+\sum_{j=j_{0}}^{J} \sum_{k \in \mathcal{K}_{j}} \alpha_{j, k}^{0} \psi_{j, k}(x),
$$

onde $\alpha_{j, k}^{0}=\left\langle f, \psi_{j, k}\right\rangle_{d G}=\int f(x) \psi_{j, k}(x) d G(x)$ e $\mathcal{K}_{j}$ é um conjunto de índices associados ao nível j. Par construir tal base, seja

$$
I_{j, k}^{0}=\left[G^{-1}\left(\frac{k-1}{2^{j}}\right), G^{-1}\left(\frac{k}{2^{j}}\right)\right)
$$


onde $j=1, \ldots, J, k=1, \ldots, 2^{j}$, com $J$ denotando a escala mais fina considerada na decomposição. Neste esquema, a medida de um intervalo $I_{j, k}^{0}$ é igual a $2^{-j}$, e os pontos são igualmente espaçados em $L_{2}(d G)$. Para uma dada realização $X_{1}, \ldots, X_{N}$, define-se:

$$
I_{j, k}=\left[X_{\left((k-1) n_{j}+1\right)}, X_{\left(k n_{j}+1\right)}\right),
$$

onde $j=1, \ldots, J, k=1, \ldots, 2^{j}, n_{j}=N 2^{-j}, j_{0} \leq j \leq J$, para algum nível inicial $j_{0}$, e denote por $X_{(1)} \leq \ldots \leq X_{(N)}$, as estatísticas de ordem. Como convenção, seja $X_{(N+1)}=$ $X_{(N)}+\left(X_{(N)}-X_{(1)}\right) / N$. Além disso, sempre que se trate de ondaletas adaptativas, os índices $j$ e $k$ iniciarão em 1 , ao invés de zero, mantendo, desse modo, a notação utilizada em Delouille (2001).

Assim, uma base ortogonal em $L_{2}(d G)$ é formada pelas funções escala não balanceadas de Haar (Girardi e Swelddens, 1997):

$$
\phi_{j, k}(x)=\frac{1}{\mu\left(I_{j, k}\right)^{1 / 2}} I\left(x \in I_{j, k}\right),
$$

e as correspondentes ondaletas

$$
\psi_{j, k}=\frac{1}{\mu\left(I_{j, m}\right)^{1 / 2}}\left(\sqrt{\frac{\mu\left(I_{j+1, l}\right)}{\mu\left(I_{j+1, l+1}\right)}} I\left(x \in I_{j+1, l+1}\right)-\sqrt{\frac{\mu\left(I_{j+1, l+1}\right)}{\mu\left(I_{j+1, l}\right)}} I\left(x \in I_{j+1, l}\right)\right),
$$

onde o índice $k$ é usado para denotar a localização dos intervalos $I_{j, k}$ tal que $I_{j, k}=I_{j+1, l} \cup$ $I_{j+1, l+1}$, para algum $l$. Na pratica, a medida $\mu$ é substituída pela medida empírica

$$
\hat{\mu}_{N}\left(I_{j, k}\right)=N^{-1} \sum_{t=1}^{N} I\left(X_{t} \in I_{j, k}\right)=2^{-j}
$$

Neste caso, as equações (1.34) e (1.35) têm forma similar às ondaletas clássicas de Haar:

$$
\begin{array}{r}
\phi_{j, k}(x)=2^{j / 2} I\left(x \in I_{j, k}\right), \\
\psi_{j, k}(x)=2^{-1 / 2}\left(\phi_{j+1,2 m}(x)-\phi_{j+1,2 m-1}(x)\right) .
\end{array}
$$

Equações análogas podem ser obtidas substituindo $I_{j, k}$ por $I_{j, k}^{o}$. 
Portanto, uma função $f(x)$ pode ser representada pela série infinita

$$
f(x)=\sum_{k=1}^{2^{j_{0}}} s_{j_{0}, k}^{o} \phi_{j_{0}, k}^{o}(x)+\sum_{j \geq j_{0}} \sum_{k=1}^{2^{j}} \alpha_{j, k}^{o} \psi_{j, k}^{o}(x),
$$

para algum nível de resolução inicial $j_{0}$, em que

$$
\begin{aligned}
\phi_{j_{0}, k}^{o}(x) & =2^{j_{0} / 2} 1_{j k}^{0}(x), \quad j=1, \ldots, J, k=1, \ldots, 2^{j} \\
\psi_{j, k}^{o}(x) & =2^{-1 / 2}\left(\phi_{j+1,2 k+1}^{0}-\phi_{j+1,2 k}^{0}\right), \quad j=1, \ldots, J-1, k=1, \ldots, 2^{j}
\end{aligned}
$$

em que $1_{j k}^{0}$ é a variável indicadora do intervalo $I_{j k}^{0}$ e

$$
\begin{aligned}
\alpha_{j, k}^{o}=\left\langle f(x), \psi_{j, k}^{0}(x)\right\rangle_{d G} & =\int f(x) \psi_{j, k}^{o} d G(x), \\
s_{j_{0}, k}^{o} & =\left\langle f(x), \phi_{j_{0}, k}^{0}(x)\right\rangle_{d G} .
\end{aligned}
$$

Se for permissível um agrupamento dos dados e se $G$ for conhecida, $\alpha_{j, k}^{o}$ pode ser estimado por

$$
\hat{\alpha}_{j, k}^{0}=N^{-1} \sum_{t=1}^{N} Y_{t} \psi_{j, k}^{o}\left(X_{t}\right),
$$

e similarmente para $s_{j_{0}, k}^{o}$. Considerando o espaço $L_{2}(d \hat{G})$, os correspondentes coeficientes empíricos de ondaleta são dados por

$$
\begin{aligned}
\hat{\alpha}_{j, k}=N^{-1} \sum_{t=1}^{N} Y_{t} \psi_{j, k}\left(X_{t}\right) & =\hat{\beta}_{j k}+\hat{\rho_{j k}} \\
& =\frac{1}{N} \sum_{t=1}^{N} f\left(X_{t}\right) \psi_{j k}\left(X_{t}\right)+\frac{1}{N} \sum_{t=1}^{N} \epsilon_{t} \psi_{j k}\left(X_{t}\right)
\end{aligned}
$$

e

$$
\hat{s}_{j_{0}, k}=N^{-1} \sum_{t=1}^{N} Y_{t} \phi_{j_{0}, k}\left(X_{t}\right)
$$

Para um conjunto de dados $\left\{X_{t}, Y_{t}\right\}_{t=1}^{N}$, o estimador não linear de $f$ em (1.24) é obtido por aplicação de limiares aos coeficientes de ondaletas, usando a base de Haar não balanceada (1.37) 
e (1.38) e limiar duro,

$$
\hat{f}(x)=\sum_{k=1}^{2^{j_{0}}} \hat{s}_{j_{0}, k} \phi_{j_{0}, k}(x)+\sum_{j=j_{0}}^{J-1} \sum_{k=1}^{2^{j}} \eta_{H}\left(\hat{\alpha}_{j, k}, t_{j, k}\right) \psi_{j, k}(x),
$$

onde $t_{j, k}$ é algum limiar.

\subsection{Processos Mixing}

As definições e teoremas que aparecem nesta seção podem ser vistos em Doukhan (1994).

Definição 1.5.1 Seja $(\Omega, \mathcal{F}, P)$ um espaço de probabilidade, e sejam $\mathcal{G}$ e $\mathcal{H}$ duas sub $\sigma$-álgebras de $\mathcal{F}$, então

$$
\alpha(\mathcal{G}, \mathcal{H})=\sup _{G \in \mathcal{G}, H \in \mathcal{H}}|P(G \cap H)-P(G) P(H)|
$$

é conhecido como coeficiente mixing forte e define uma medida de depêndencia entre $\mathcal{G}$ e $\mathcal{H}$.

Se as sub $\sigma$-álgebras $\mathcal{G}$ e $\mathcal{H}$ são independentes, então $\alpha(\mathcal{G}, \mathcal{H})=0$

Definição 1.5.2 Considere a sequência de variáveis aleatórias $\left\{X_{t}, t \in \mathbb{Z}\right\}$ não necessariamente estacionária e sejam

$$
\mathcal{F}_{t}=\mathcal{F}_{-\infty}^{t}=\sigma\left(\ldots, X_{t-2}, X_{t-1}, X_{t}\right) \quad e \quad \mathcal{F}_{t+m}^{+\infty}=\sigma\left(X_{t+m}, X_{t+m+1}, \ldots\right)
$$

A sequência $\left\{X_{t}\right\}$ é dita ser fortemente mixing ou $\alpha-$ mixing se

$$
\lim _{m \rightarrow \infty} \alpha_{m}=0
$$

em que

$$
\alpha_{m}=\sup _{t} \alpha\left(\mathcal{F}_{-\infty}^{t}, \mathcal{F}_{t+m}^{+\infty}\right)
$$

Se o processo $X_{t}$ é fortemente estacionário então $\alpha_{m}=\alpha\left(\mathcal{F}_{-\infty}^{0}, \mathcal{F}_{m}^{\infty}\right)$. Já que as coleções $\mathcal{F}_{-\infty}^{t}$ e $\mathcal{F}_{t+m}^{+\infty}$ são, respectivamente, não decrescente e não crescente em $t$ e $m$, a sequênça $\left\{\alpha_{m}\right\}_{0}^{\infty}$ é monótona. O termo tamanho da sequência descreve a taxa de convergência dos $\alpha_{m}$. Uma sequência é $\alpha-$ mixing de tamanho $-\varphi_{0}$ se $\alpha_{m}=O(m)^{-\varphi}$ para algum $\varphi>\varphi_{0}$.

Observe que mixing não é somente uma propriedade da sequência $\left\{X_{t}\right\}$ mas também das $\sigma$-álgebras geradas pelas sequências, o que implica que a propriedade mixing se conserva para qualquer variável aleatória mensurável nessas $\sigma$-álgebras. 
Teorema 1.5.1 Seja $Y_{t}=g\left(X_{t}, X_{t-1}, \ldots, X_{t-k}\right)$ uma função mensurável para qualquer $k$ inteiro positivo. Se $X_{t}$ é $\alpha$-mixing de tamanho $-\alpha$, então $Y_{t}$ também o é.

\subsection{Dependência Fraca}

As definições e resultados apresentados nesta seção podem ser estudados com mais detalhe em Dedecker et al. (2007).

Definição 1.6.1 Uma sequência $\left\{X_{t}\right\}$ é dita $(\theta, \mathcal{L}, \psi)$-fracamente dependente se existe uma classe $\mathcal{L}$ de funções de valor real, uma sequência dependente $\theta=\left(\theta_{r}\right)_{r \in \mathbb{R}}$ decrescendo a zero em infinito, e uma função $\psi$ com argumentos $(h, k, u, v) \in\left(\mathcal{L}^{2} \times \mathbb{N}^{2}\right)$, tal que para qualquer u-upla $\left(i_{1}, i_{2}, \ldots, i_{u}\right)$ e para qualquer v-upla $\left(j_{1}, j_{2}, \ldots, j_{v}\right)$, com $i_{1} \leq i_{2}, \ldots, \leq i_{u} \leq j_{1} \leq j_{2} \leq \ldots \leq j_{v}$, se tem

$$
\left|\operatorname{Cov}\left(h\left(X_{i_{1}}, \ldots, X_{i_{u}}\right), k\left(X_{j_{1}}, \ldots, X_{j_{v}}\right)\right)\right| \leq \psi(h, k, u, v) \theta_{r}
$$

com as funções $h, k \in \mathcal{L}$ e definidas em $\mathbb{R}^{u}$ e $\mathbb{R}^{v}$, respectivamente.

Definição 1.6.2 Seja $\left\{X_{t}\right\}$ uma sequência de variáveis aleatórias centradas (com valor esperado zero). Para um inteiro positivo $r$ é definido o coeficiente de dependência fraca como uma sequência não decrescente $\left(C_{r, q}\right)_{q \geq 2}$ tal que

$$
\sup \left|\operatorname{Cov}\left(X_{t_{1}} \ldots X_{t_{m}}, X_{t_{m+1}} \ldots X_{t_{q}}\right)\right|:=C_{r, q}
$$

em que o supremo é tomado sobre todas as $\left\{t_{1}, t_{2}, \ldots, t_{q}\right\}$ tal que $t_{1} \leq \ldots \leq t_{q}$ e $m$ e r satisfazem $t_{m+1}-t_{m}=r$.

Lema 1.6.1 (Doukhan e Louhichi (1999)). Se a sequência $\left\{X_{t}\right\}, t \in \mathbb{Z}$, é fortemente mixing, então esta é $\left(\alpha, \mathcal{L}^{\infty}, \psi\right)$ fracamente dependente com $\psi(h, k, u, v)=4\|h\|_{\infty}\|k\|_{\infty}$. Além disso, se a sequência tem valor esperado zero e é limitada por $M$, então

$$
C_{r, q} \leq 4 M^{q} \alpha_{r}
$$

Condições 1.6.1 As seguintes condições devem ser satisfeitas para poder aplicar o teorema e corolário que vem a seguir:

1.

$$
\left|\operatorname{Cov}\left(f\left(X_{1}, \ldots, X_{n}\right), g\left(X_{1}, \ldots, X_{n}\right)\right)\right| \leq \sum_{i \in I} \sum_{j \in J}\left\|\frac{\partial f}{\partial x_{i}}\right\|_{\infty}\left\|\frac{\partial g}{\partial x_{j}}\right\|_{\infty}\left|\operatorname{Cov}\left(X_{i}, X_{j}\right)\right|
$$


em que $f$ e $g$ são funções de valores reais definidas em $\mathbb{R}^{n}$, com primeira derivada limitada e dependendo de $x_{i} \in I$ e $x_{j} \in J$, respectivamente, I e $J$ são subconjuntos de $\mathbb{N}$.

2.

$$
C_{r, q} \leq C M^{q} \epsilon_{r}
$$

com $\epsilon_{r}$ um coeficiente de dependência fraca.

3.

$$
M_{m, r}=N \sum_{r=0}^{N-1}(r+1)^{m-2} C_{m, r} \leq A_{n} \frac{m !}{\beta^{m}}
$$

em que $\beta$ é uma constante positiva e $A_{n}$ é uma sequência independente de $q$.

Teorema 1.6.1 Seja $r>0$ um número real. Seja $\left\{X_{t}\right\}$ uma sequência estritamente estacionária com valor esperado zero, que satisfaz a condição (1.53). Além disso, suponha que a sequência é limitada por $M$. Então existe uma constante positiva $C_{r}$ dependendo só de $r$ tal que

$$
E\left|S_{N}\right|^{r}=C_{r}\left(s_{N}^{r}+\sum_{k=1}^{n} \sum_{i=0}^{k-1} M^{r-2}(i+1)^{r-2}\left|\operatorname{cov}\left(X_{1}, X_{1+i}\right)\right|\right),
$$

onde $s_{N}^{r}=N \sum_{i=0}^{N}\left|\operatorname{cov}\left(X_{1}, X_{1+i}\right)\right|$ e $S_{N}=X_{1}+X_{2}+\ldots+X_{N}$.

Corolário 1.6.1 Suponha que as condições (1.54) e (1.55) são satisfeitas para alguma sequência $A_{N} \geq 1$ e para $N \geq 2$. Então existe um número real positivo $x$ tal que

$$
P\left(\left|S_{N}\right| \geq x \sqrt{A_{n}}\right) \leq A \exp (-B \sqrt{\beta x})
$$

\subsection{Função de Hölder}

As definições e propriedades apresentadas a seguir podem ser estudadas com detalhe em Cai e Brown (1998).

Uma função de Hölder por partes pode ser considerada como a sobreposição de uma função regular em uma classe de Hölder e uma perturbação que consiste em um salto de descontinuidade.

Definição 1.7.1 Uma função de Hölder por partes em $[0,1]$ com ao menos $m$ saltos de descontinuidade consiste de funções $f$ satisfazendo as seguintes condições: 
1. a função $f$ é limitada por $B$, ou seja, $|f| \leq B$;

2. Existem $l \leq m$ pontos $0 \leq a_{1}<\ldots<a_{l} \leq l$, tal que para todo $a_{i}<x, y<a_{i+1}$, $i=0,1, \ldots, l\left(\right.$ com $a_{0}=0$ e $\left.a_{l+1}=1\right)$,

- $|f(x)-f(y)| \leq M|x-y|^{\alpha}$ se $\alpha \leq 1$;

- $\left|f(x)^{\lfloor\alpha\rfloor}-f(y)^{\lfloor\alpha\rfloor}\right| \leq M|x-y|^{\alpha^{\prime}}$ e $|f(x)| \leq B$ se $\alpha>1$

onde $\lfloor\alpha\rfloor$ é o maior inteiro menor que $\alpha$ e $\alpha^{\prime}=\alpha-\lfloor\alpha\rfloor$.

A classe de funções $\Lambda^{\alpha}(M, B, m)$ consiste de funções Hölder por partes com $m$ pontos de descontinuidades.

Lema 1.7.1 Seja $f \in \Lambda^{\alpha}(M, B, m)$, suponha que a função de ondaleta $\psi$ é $r$-regular com $r \geq \alpha$. Então:

- se $\operatorname{supp}\left(\psi_{j k}\right)$ não contém pontos de descontinuidade de $f$, então $\alpha_{j k}=\left\langle f, \psi_{j k}\right\rangle \leq C 2^{-j(1 / 2+\alpha)}$,

- Se $\operatorname{supp}\left(\psi_{j k}\right)$ contém pelo menos um ponto de descontinuidade em $f$, então $\alpha_{j k}=\left\langle f, \psi_{j k}\right\rangle \leq$ $C 2^{-j / 2}$.

Agora vamos supor que temos uma função amostral diádica $\{f(k / N)\}_{k=1}^{N} \operatorname{com} N=2^{J}$. Usamos uma base de ondaleta e a análise de resolução associada para obter uma boa aproximação de f.

Lema 1.7.2 Suponha $f \in \Lambda^{\alpha}(M, B, m)$, seja $s_{j k}=\left\langle f, \phi_{j k}\right\rangle$ e $s(\alpha)=\min (\alpha, 1)$. Então:

- se $\operatorname{supp}\left(\phi_{j k}\right)$ não tem pontos de descontinuidade de $f$, então

$$
\left|N^{1 / 2} f(k / N)-s_{j k}\right| \leq C N^{-(1 / 2+s(\alpha))}
$$

- se $\operatorname{supp}\left(\phi_{j k}\right)$ tem pontos de descontinuidade da função $f$, então

$$
\left|N^{1 / 2} f(k / N)-s_{j k}\right| \leq C N^{-1 / 2}
$$





\section{Capítulo 2}

\section{Modelo de Regressão com Variáveis Fortemente Mixing Usando Ondaletas Deformadas}

\subsection{Introdução}

Neste capítulo são apresentados alguns resultados em regressão não paramétrica, usando o método de ondaleta deformada, quando a variável explicativa $X_{t}$ apresenta uma estrutura de dependência $\alpha$-mixing. O objetivo é estimar o sinal minimozando o risco. Mostra-se que, sob algumas condições específicas, pode-se estimar o sinal sob uma base deformada por $G$, função de distribuição de $X_{t}$, alcançando uma taxa de convergência quase ótima. Algumas simulações foram feitas para avaliar o desempenho da metodologia.

\subsection{Procedimento usando Ondaletas Deformadas}

Sejam $\left(X_{1}, Y_{1}\right), \ldots,\left(X_{N}, Y_{N}\right)$, pares de variáveis aleatórias observadas, com $N=2^{J_{0}}$. Considere o modelo:

$$
Y_{t}=f\left(X_{t}\right)+\epsilon_{t}, \text { para } t=1,2, \ldots, N
$$

em que $f$ é uma função desconhecida e $\epsilon_{t}$ é um termo de erro. Para estimar $f$ será utilizada a metodologia de ondaletas deformadas apresentada no Capítulo 1. O objetivo é estimar a função $f$ por $\hat{f}$, com baixo risco

$$
R(f, \hat{f})=E\|f-\hat{f}\|_{p}^{p}=E\left(\int|f(x)-\hat{f}(x)|^{p} d x\right) \text { para, } 1 \leq p<\infty
$$

para alguma ampla classe de funções. Para alcançar este objetivo de risco mínimo, fazemos as seguintes suposições: 
1. A função $f$ é limitada e possui suporte compacto no intervalo $[a, b]$.

2. $X_{t}$ é uma sequência de variáveis aleatórias estacionárias com média zero, variância $\sigma^{2}<\infty$, $\operatorname{cov}\left(X_{t}, X_{r}\right)=\gamma_{t, r}=\gamma|t-r|$, para todo $r, t \in \mathbb{Z}$ e $\gamma \in \mathbb{R}$

3. $X_{t}$ tem função de distribuição $G(x)$ e respectiva densidade $g(x)$ com suporte no intervalo $[a, b]$, (o mesmo intervalo de suporte de $f$ ).

4. A função de distribuição $G(x)$ e sua inversa $G^{-1}(x)$ são contínuas e estritamente monótonas, ou seja, $G\left(G^{-1}(x)\right)=x$ q.c e $G^{-1}(G(x))=x$ q.c, para todo $x \in[a, b]$.

5. A função $\omega(x)=\left[g\left(G^{-1}(x)\right)\right]^{-1}$ é um peso de Muckenhoupt: $\omega \in A_{p}([a, b])$. Se $0<g<$ $M<\infty$, então $\omega(x) \in A_{\infty}$.

6. Os erros $\left\{\epsilon_{t}\right\}_{t \in \mathbb{Z}}$ são variáveis aleatórias independentes e identicamente distribuídas (i.i.d.) com $E\left[\epsilon_{t}\right]=0, E\left[\epsilon_{t}^{2}\right]=\sigma_{\epsilon}^{2}<\infty$ e $\epsilon_{t}$ é independente de $X_{s}$, para todo $t, s \in \mathbb{Z}$

7. Existem $c \geq p, c \in 2 \mathbb{N}$ e $\delta>0$, que satisfazem as condições mixing

$$
\sum_{h=1}^{\infty}(h+1)^{c-2}\left(\alpha_{X_{t}, h}\right)^{\frac{\delta}{(\delta+c)}}<\infty
$$

para coeficientes mixing forte $\alpha_{X_{t}, h}$, como definido em Doukhan (1994).

A função $f$ será estimada por meio de métodos de ondaletas por:

$$
\hat{f}=\sum_{j=-1}^{J} \sum_{k=0}^{2^{j}-1} \hat{\beta}_{j, k} \eta_{H}\left(\hat{\beta}_{j, k}, \kappa t_{N}\right) \psi_{j, k}(G(x))
$$

para algum $\kappa>0, \operatorname{com} \eta_{H}(d, \lambda)=d, I_{|d| \geq \lambda}, \lambda=k t_{N} \mathrm{e}$

$$
\begin{gathered}
t_{N}=\left(\frac{\log N}{N}\right)^{1 / 2}, \\
\hat{\beta}_{j, k}=\frac{1}{N} \sum_{t=1}^{N} \psi_{j, k}\left(G\left(X_{t}\right)\right) Y_{t} .
\end{gathered}
$$

Se a função $G$ não for conhecida, é usada a distribuição empírica. Os novos estimadores serão denotados por $\tilde{\beta}_{j, k}$ e $\tilde{f}(x)$. 


\subsection{Resultados Principais}

Seja $\beta_{j, k}$ os coeficientes da função $f\left(G^{-1}(y)\right)$ na base de ondaletas $\left\{\psi_{j, k}, j \geq-1, k=0, \ldots, 2^{j}-1\right\}$,

$$
f\left(G^{-1}(y)\right)=\sum_{j=0}^{\infty} \sum_{k=0}^{2^{j}-1} \beta_{j, k} \psi_{j, k}\left(G^{-1}(y)\right),
$$

onde f é estimada por ondaletas deformadas, conforme (2.4). Para estudar a taxa de convergência do procedimento, será utilizada a avaliação de conjuntos maximais. Esta avaliação é um modo de se mensurar o desempenho de procedimentos estatísticos segundo Kerkyacharian e Picard (2004). Possui a vantagem de fornecer comparações de procedimentos menos pessimistas do que a abordagem minimax. Porém, enquanto o risco minimax pode ser calculado em muitas situações para um determinado tamanho de amostra, a noção de conjunto maximal está associada a uma sequência de procedimentos estatísticos e a uma taxa de convergência. Portanto, é de natureza puramente assintótica.

Para demonstrar os resultados a partir desta abordagem são necessárias as seguintes definições:

Definição 2.3.1 Considere uma sequência de modelos $\epsilon_{N}=\left\{P_{\theta}^{N}, \theta \in \Theta\right\}$, em que $P_{\theta}^{N}$ são distribuições de probabilidade e $\Theta$ é o espaço paramétrico. Considere também uma sequência $\hat{q}_{N}$ de estimadores de uma quantidade $q(\theta)$, uma função de perda $L\left(\hat{q}_{N}, q(\theta)\right)$ e uma taxa de convergência $a_{N}$ tendendo a zero. Então, o conjunto maximal associado com a sequência $\hat{q}_{N}$, a função de perda L, a taxa $a_{N}$ e uma constante $T$, é:

$$
\operatorname{Max}\left(\hat{q}_{N}, L, a_{N}\right)(T)=\left\{\theta \in \Theta, \sup _{N} L\left(\hat{q}_{N}, q(\theta)\right) a_{N}^{-1} \leq T\right\}
$$

Em particular, o conjunto maximal associado ao estimador $\hat{f}$, obtido aplicando ondaletas deformadas é:

$$
\operatorname{Max}\left(\hat{f}, L,\left(t_{N}\right)^{(p-q)}\right)(\infty)=\left\{f, E\|\hat{f}-f\|_{p}^{p}\left(t_{N}\right)^{(q-p)}<\infty\right\}
$$

Definição 2.3.2 Para $p>1, j \in \mathbb{N}, k \in \mathbb{Z}$, seja $\nu$ a medida

$$
\nu\{(j, k)\}=2^{j p / 2} \omega\left(I_{j, k}\right),
$$


em que $\omega$ é um peso de Muckenhoupt e $I_{j, k}$ é um intervalo qualquer da reta indexado por $j$ e $k$. Usando esta medida, é definido o seguinte espaço de funções:

$$
l_{q, \infty}(\nu)=\left\{f(x)=\sum_{j \geq-1} \sum_{k \in \mathbb{Z}} \beta_{j, k} \psi_{j, k}(G(x)), \sup _{t>0} t^{q} \nu\left\{(j, k):\left|\beta_{j, k}\right|>t\right\}<\infty\right\} .
$$

A seguinte proposição apresenta algumas propriedades dos estimadores dos coeficientes de ondaletas.

Proposição 2.3.1 Para qualquer $j \geq 0$, e qualquer $k=0,1, \ldots, 2^{j}-1$, sob as suposições 1 a 6, o estimador (2.6) satisfaz as seguintes propriedades:

(a) $E\left[\hat{\beta}_{j, k}\right]=\beta_{j, k}$.

(b)

$$
\begin{aligned}
\operatorname{cov}\left[\hat{\beta}_{j, k} ; \hat{\beta}_{j^{\prime}, k^{\prime}}\right] & =N\left(8 \alpha_{W_{|s-t|}}^{1 / r}\|f\|_{\infty} 2^{j\{(p-2) / 2 p+(q-2) / 2 q\}}\|\psi\|_{\infty}^{(p-2) / p+(q-2) / q}\right) \\
& +\sigma_{\epsilon}^{2} \int_{a}^{b} \psi_{j, k}(G(X)) \psi_{j^{\prime}, k^{\prime}}(G(X)) g(x) d x \\
& +\sum_{t=1}^{N} \sum_{s \neq t}^{N}\left(8 \alpha_{W_{|s-t|}^{1 / r}}^{1 / r}\|f\|_{\infty} 2^{j\{(p-2) / 2 p+(q-2) / 2 q\}}\|\psi\|_{\infty}^{(p-2) / p+(q-2) / q}\right),
\end{aligned}
$$

$\operatorname{com} W_{t, j, k}=\psi_{j, k}\left(G\left(X_{t}\right)\right) f\left(X_{t}\right)$.

(c) $\operatorname{Var}\left[\hat{\beta}_{j, k}\right] \leq N^{-1}\left\{\|f\|_{\infty}^{2}+\sigma_{\epsilon}^{2}-\beta_{j, k}^{2}+8 \alpha_{w_{t, h}}^{1 / r}\|f\|_{\infty} 2^{(j / 2)\{(p-2) / p+(q-2) / q\}}\right\}$,

com $p, q, r \geq 1$ e $1 / p+1 / q+1 / r=1$.

Baseado no Teorema 1 de Kerkyacharian e Picard (2004), é possível garantir que as ondaletas deformadas satisfazem às propriedades de encolhimento e $p$-Temlyakov. Sob as suposições 1 a 7, o Teorema 5, dos mesmos autores, informa que:

$$
\sup _{N} \nu\left\{\mathcal{J}_{N}\right\}\left(\frac{\log N}{N}\right)^{p / 2}<\infty
$$

onde $\mathcal{J}_{N}=\left\{(j, k):|k| \leq n 2^{j}\right\}$, para $0 \leq j \leq J$, e onde $\mathrm{n}$ é o comprimento do suporte da ondaleta mãe $\psi$.

Teorema 2.3.1 Seja $0<q<p<\infty, p>1$ e considere $\left\{\psi_{j, k}, j \geq 1, k \in \mathbb{N}\right\}$ satisfazendo as propriedades 1 e 2. Suponha $t_{N}$ uma sequência de números reais tendendo a zero, e $\mathcal{J}_{N}$ um 
conjunto de pares tal que

$$
\operatorname{sup\nu }_{N}\left(\mathcal{J}_{N}\right) t_{N}^{p}<\infty
$$

adicionalmente, suponha que para todo $(j, k) \in \mathcal{J}_{N}$, existe um estimador $\hat{\beta}_{j k}$ tal que

$$
\begin{aligned}
& E\left\|\hat{\beta}_{j k}-\beta_{j k}\right\|^{2 p} \leq C t_{N}^{2 p} \\
& P\left(\left|\hat{\beta}_{j k}-\beta_{j k}\right|>\frac{\mathcal{K} t_{N}}{2}\right) \leq C_{p} N^{-\gamma p} .
\end{aligned}
$$

Se $\hat{f}$ é definido como em (2.4), então para $s=(1 / q-1 / p) p / 2$ existe $A=A(p, q, k, C)$, tal que $E\|\hat{f}-f\|_{p}^{p} \leq t_{N}^{2 s p /(1+2 s)} A\left(\|f\|_{l_{q, \infty}}^{q}+t_{N}^{p+q}\left[\|f\|_{p}^{p}+\nu\left\{(j, k) \in \mathcal{J}_{N}\right\}\right]\right)+\left\|\sum_{(j, k) \notin \mathcal{J}_{N}} \beta_{j k} \psi_{j k}(G(x))\right\|_{p}^{p}(2$

O teorema 2.3.1 é um caso particular do Teorema 5.1 em Kerkyacharian e Picard (2000), onde são consideradas de forma geral bases incondicionais (bases que satisfazem as condições (1.29)) e (1.30). A prova é apresentada no final deste capítulo.

Corolário 2.3.1 Sob as suposições do Teorema 2.3.1 e

a) $\mathcal{J}_{N}=\left\{(j, k) ;|k| \leq n 2^{j},-1 \leq j \leq J\right\}$ e $2^{J} \sim\left(t_{N}\right)^{-r}$,

b) $\|f\|_{l_{q, \infty}} \leq C_{1}$,

c) $f \in B_{p, \infty}^{2 s p /(1+2 s)}\left(\psi_{j k}\right)$,

então

$$
E\|\hat{f}-f\|_{p}^{p} \leq C t_{N}^{2 s p /(1+2 s)}
$$

A proposição apresentada a seguir permite garantir que são satisfeitas as condições do Teorema 2.3.1, quando variáveis dependentes são consideradas no desenho.

Proposição 2.3.2 Seja $0<C<\infty$ uma constante que não depende do tamanho da amostra $N$. Seja $t_{N}=\left(\frac{\log N}{N}\right)^{1 / 2}$. Então, para qualquer $j=0,1, \ldots, J_{1}, 2^{J_{1}} \leq t_{N}$ e qualquer $k=$ $0, \ldots, 2^{J_{1}}-1$, sob as suposições 1 a 6 , o estimador (2.6) satisfaz: 
a)

$$
E\left[\left|\hat{\beta}_{j, k}-\beta_{j, k}\right|^{m}\right] \leq C t_{N}^{2 m}
$$

b)

$$
P\left(\left|\hat{\beta}_{j, k}-\beta_{j, k}\right|>\mathcal{K} t_{N}\right) \leq C_{p} N^{-\vartheta p}
$$

$\operatorname{com} \vartheta=-\frac{B}{4 N^{3 / 2}\|\psi\|_{\infty}}$.

O teorema apresentado a seguir permite concluir que

$$
\operatorname{Max}\left(\hat{f}, L_{p}, t_{N}^{p-q}\right)(\infty) \subseteq l_{q, \infty}(\nu) \bigcap B_{\infty}^{2 s p /(1+2 s)},
$$

onde, se $f \in B_{\infty}^{2 s p /(1+2 s)}$, então $\sup _{N}\left(t_{N}\right)^{2 s p /(1+2 s)}\left\|f-\sum_{(j, k) \in \mathcal{J}_{N}} \beta_{j k} \psi_{j k}\right\| \leq \infty$.

Teorema 2.3.2 Seja $s>0, r>0, p>1,\left\{\psi_{j, k}, j \geq 1, k \in \mathbb{N}\right\}$ satisfazendo as propriedades 1 e 2. Suponha $\mathcal{J}_{N}=\left\{(j, k):|k| \leq n 2^{j}\right\}$, um conjunto de pares, tal que $2^{J} \sim t_{N}^{-r}$, onde $t_{N}=\left(\frac{\log N}{N}\right)^{1 / 2}$. Adicionalmente, considere a sequência de experimentos $E_{N}$, tal que para todo $n \in \mathbb{N}^{*}$

$$
E\|\hat{f}-f\|_{p}^{p} \leq C t_{N}^{2 s p /(1+2 s)}
$$

onde $\hat{f}$ está definido em (2.4). Então, para $q$ tal que $s=(1 / q-1 / p) p / 2$,

$$
f \in l_{q, \infty} \bigcap B_{\infty}^{2 s p /(1+2 s)} .
$$

Teorema 2.3.3 Seja $p>1,0<q<p$. Sob as suposições 1 a 7 , o conjunto maximal associado ao estimador $\hat{f}$, definido em (2.9), pode ser expresso como:

$$
\begin{aligned}
& \operatorname{Max}\left(\hat{f}, L_{p},\left(t_{N}\right)^{p-q}\right)(\infty)=l_{q, \infty}(\nu) \cap\left\{f(x)=\sum_{j \geq 0} \sum_{k \in \mathbb{Z}} \beta_{j, k} \psi_{j, k}(G(x)),\right. \\
& \left.\sup _{l>0}\left\|\sum_{j \geq 0} \sum_{k \in \mathbb{Z}} \beta_{j, k} \psi_{j, k}(G(x))\right\|_{p}^{p} 2^{l(p-q)}<\infty\right\} .
\end{aligned}
$$

Este teorema é o equivalente ao teorema 3 de Kerkyacharian e Picard (2004). 


\subsection{Simulações}

Algumas simulações foram feitas para avaliar o efeito do tamanho da amostra N, o nível do ruído e a escolha da base de ondaleta. As simulações foram feitas usando o programa computacional MATLAB e os pacotes de WAVELAB850 e PROGREG (fornecido por Chesneau e Willer (2007)).

Os dados $\left(x_{1}, y_{1}\right), \ldots,\left(x_{N}, y_{N}\right)$ foram gerados a partir de um modelo da forma (2.1), onde $X_{t}$ segue um processo $\operatorname{AR}(1)$ da seguinte forma:

$$
X_{t}=0,2 X_{t-1}+u_{t} \quad \text { e } \quad Y_{t}=f\left(X_{t}\right)+\epsilon_{t}
$$

$u_{t}$ tem densidade conhecida, e $\epsilon_{t}$ tem distribuição normal padrão e $N=512,1024$ e 2048 . As bases das ondaletas usadas são Symmlet8 e Coiflet3 em todos os casos.

A amostra da variável $X_{t}$ foi obtida do seguinte modo: tomando $x_{0}=0$ é gerado, a seguir, um valor de $u_{t}$, com uma densidade g conhecida, pelo método de aceitação-rejeição. Logo, é feito

$$
x_{1}=0,2 x_{0}+u_{t}
$$

Depois é gerado um valor $u_{2}$, de novo pelo método de aceitação-rejeição e é feito $x_{2}=0,2 x_{1}+u_{2}$, assim sucessivamente até gerar 3000 valores de $x_{t}$. Na sequência, são escolhidos os últimos $\mathrm{N}$ valores, para $N=512,1024,2048$ (segundo cada caso), e os outros valores são descartados. Finalmente, os valores de $x_{t}$ são ordenados e os valores de $y_{t}$ são calculados sem ruído. Estes pontos foram gerados uma única vez, tornando-os fixos nas simulações; também são gerados os ruídos $\epsilon_{t}$ com distribuição $\mathcal{N}(0,1)$ e adicionados aos valores $y_{t}$.

Três funções $f$ foram consideradas como teste, representando diferentes graus de variabilidade espacial:

1 Seno: $f(x)=0,2+0.6 \operatorname{sen}(\pi x)$,

2 Heavisine: $f(x)=4 \operatorname{sen}(4 \pi x)-\operatorname{sgn}(x-0,3)-\operatorname{sgn}(0,72-x)$,

3 Doppler: $\{x(1-x)\}^{(1 / 2)} \operatorname{sen}\{2 \pi(1+\delta) /(x+\delta)\}, \delta=0,05$.

As duas últimas expressões estão em Donoho e Johnstone (1994), reescaladas verticalmente 


\begin{tabular}{|c|c|c|c|c|c|c|c|c|}
\hline \multirow[b]{2}{*}{$\mathrm{N}$} & \multicolumn{5}{|c|}{$\mathrm{SNR}=1$} & \multicolumn{3}{|c|}{$\mathrm{SNR}=7$} \\
\hline & $j_{0}$ & Symmlet8 & $j_{0}$ & Coiflet3 & $\%$ dif & Symmlet8 & Coiflet3 & \%dif \\
\hline \multicolumn{9}{|c|}{ Seno-Constante } \\
\hline 512 & 5 & 0,0860 & 4 & 0,0929 & 89 & 0,0810 & 0,0813 & 14 \\
\hline 1024 & 6 & 0,0736 & 5 & 0,0771 & 89 & 0,0699 & 0,0772 & 33 \\
\hline 2048 & 7 & 0,0821 & 6 & 0,0839 & 89 & 0,0783 & 0,0804 & 78 \\
\hline \multicolumn{9}{|c|}{ Seno-Seno } \\
\hline 512 & 5 & 0,0836 & 4 & 0,0899 & 88 & 0,0766 & 0,0768 & 3 \\
\hline 1024 & 6 & 0,0817 & 5 & 0,0851 & 87 & 0,0785 & 0,0786 & 47 \\
\hline 2048 & 7 & 0,0783 & 6 & 0,0802 & 84 & 0,0771 & 0,0770 & 40 \\
\hline \multicolumn{9}{|c|}{ HeaviSine-Constante } \\
\hline 512 & 5 & 0,1185 & 4 & 0,1238 & 85 & 0,1154 & 0,1155 & 12 \\
\hline 1024 & 6 & 0,1008 & 5 & 0,1058 & 87 & 0,1013 & 0,1015 & 15 \\
\hline 2048 & 7 & 0,1102 & 6 & 0,1129 & 90 & 0,1115 & 0,115 & 37 \\
\hline \multicolumn{9}{|c|}{ HeaviSine-Seno } \\
\hline 512 & 5 & 0,0810 & 4 & 0,0886 & 89 & 0,0769 & 0,0772 & 7 \\
\hline 1024 & 6 & 0,0996 & 5 & 0,1033 & 38 & 0,0987 & 0,0989 & 11 \\
\hline 2048 & 7 & 0,1249 & 6 & 0,1266 & 54 & 0,1255 & 0,1257 & 25 \\
\hline \multicolumn{9}{|c|}{ Doppler-Constante } \\
\hline 512 & 5 & 0,1737 & 4 & 0,1839 & 82 & 0,1547 & 0,1843 & 2 \\
\hline 1024 & 6 & 0,1779 & 5 & 0,1824 & 88 & 0,1824 & 0,1825 & 39 \\
\hline 2048 & 7 & 0,1696 & 6 & 0,1714 & 82 & 0,1716 & 0,1716 & 39 \\
\hline \multicolumn{9}{|c|}{ Doppler-Seno } \\
\hline 512 & 5 & 0,1600 & 4 & 0,1595 & 41 & 0,1567 & 0,1567 & 4 \\
\hline 1024 & 6 & 0,1754 & 5 & 0,1789 & 79 & 0,1787 & 0,1789 & 25 \\
\hline 2048 & 7 & 0,1776 & 6 & 0,1794 & 56 & 0,1809 & 0,806 & 38 \\
\hline
\end{tabular}

Tabela 2.1: RMSE do estudo de simulação. As colunas com "\%dif" são as diferenças percentuais entre o RMSE da base symmlet 8 e coiflet 3 . Foi atribuído o valor zero quando a diferença era insignificante com nivel $5 \%$, com o teste de Wilcox para amostras dependentes.

para $[0,2 ; 0,8]$.

As funções de densidade a testar são:

1 Constante: $g(x)=\mathbf{1}(0 \leq x \leq 1)$,

2 Seno: $g(x)=1+0,2 \operatorname{sen}(4 \pi x)$.

Como em Chesneau e Willer (2007), escolhe-se o valor da constante $k=1$ e são comparados os estimadores em dois níveis de ruído: $s n r=1$ (ruído maior) e $s n r=7$ (ruído menor), onde

$$
s n r=\frac{(N-1)^{-1} \sum_{t=1}^{N}\left(f\left(x_{t}\right)-\bar{f}\right)^{2}}{V\left(\sigma_{\epsilon}^{2}\right)}
$$

e $\bar{f}=N^{-1} \sum_{t=1}^{N} f\left(x_{t}\right)$. Foram consideradas amostras de tamanho $\mathrm{N}=512,1024$ e 2048.

A Tabela 2.1 apresenta os RMSE sobre 200 replicações das funções testadas. As bases de ondaletas usadas foram Symmlet de ordem 8 e Coiflet de ordem 3. Os coeficientes foram limia- 
rizados de modo duro a partir do nível $j_{0}$ indicado até a escala mais fina. O teste dos postos sinalizados de Wilcox foi usado para testar a significância das diferenças entre os RMSE da estimação com a base symmlet e a base coiflet. Quando a diferença não era significativa a 5\%, foi atribuido o valor zero.

Para $f(x)$ sendo as funções Seno e Heavisine, com densidade seno, temos em geral o RMSE do estimador com base Symmlet menor para ambos níveis de ruído. Com as simulações, os melhores ajustes foram alcançados para a função $f$ sendo Seno (com as duas funções de densidade estudadas) e Heavisine com densidade $g(x)$ sendo Seno e $N=512$, enquanto o RMSE é maior para a função Doppler.
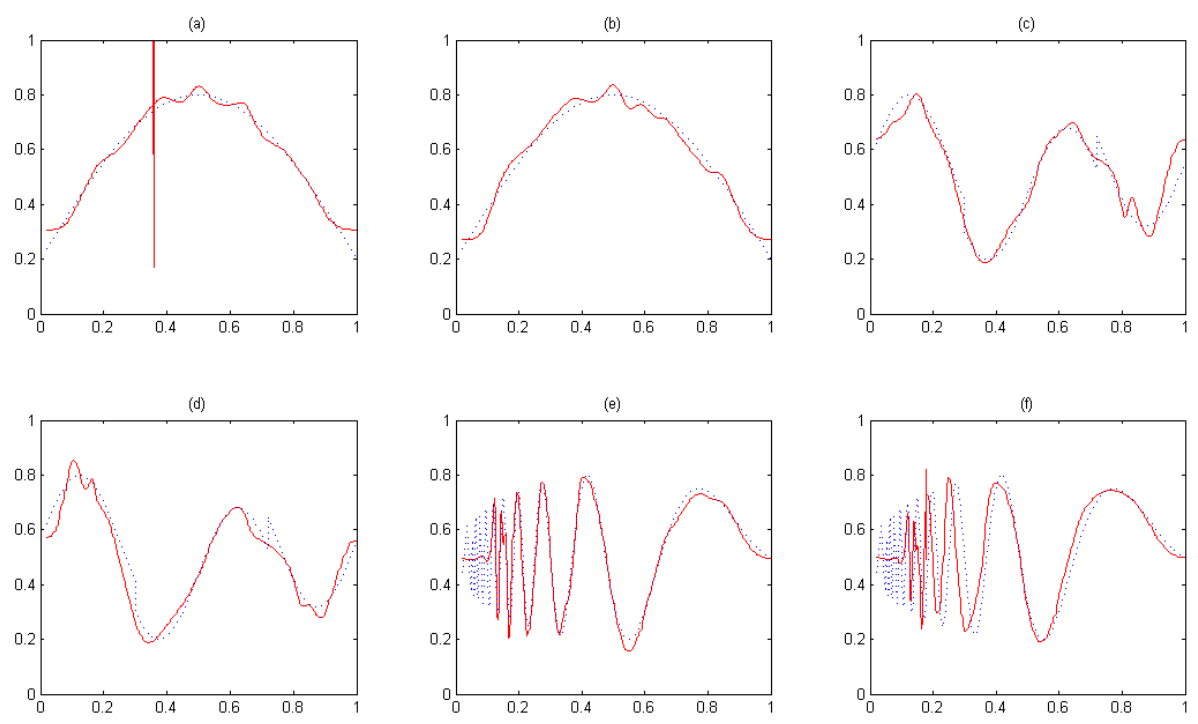

Figura 2.1: Funções (a) $f(x)$ seno e $g(x)$ constante, (b) $f(x)$ seno e $g(x)$ seno, (c) $f(x)$ Heavisine e $g(x)$ constante, (d) $f(x)$ Heavisine e $g(x)$ seno, (e) $f(x)$ Doppler e $g(x)$ constante, (f) $f(x)$ Doppler e $g(x)$ seno; com $\mathrm{N}=2048$ e rsn=1. As estimativas (linhas cheias) foram calculadas com a base symmlet 8 . A função teórica é dada pelas linhas tracejadas.

Na Figura 2.1 são apresentadas as estimativas para as funções Seno, Heavisine e Doppler com funções de densidade constante e seno, no caso de $\mathrm{N}=2048$ e rsn=1. Observa-se que a metodologia de ondaletas deformadas tem um bom comportamento nas estimativas para ambas funções de densidade, bem como para as funções f com menos variabilidade espacial (como é o 
caso de Seno e HeaviSine).

A qualidade das estimativas foi avaliada pelo cálculo aproximado da raiz do erro quadrático médio integrado (RMSE) e o desvio máximo (MXDV). O MXDV reflete a amplitude do erro localizado na estimativa. As quantidades foram calculadas da seguinte forma:

1) RMSE é calculado como o valor médio de 200 réplicas de

$$
\sqrt{\frac{1}{N} \sum_{i=1}^{N}\left(f\left(\frac{i}{N}\right)-\hat{f}\left(\frac{i}{N}\right)\right)^{2}}
$$

2) MXDV é calculado como a média das 200 réplicas do valor

$$
\max _{1 \leq i \leq N}\left|f\left(\frac{i}{N}\right)-\hat{f}\left(\frac{i}{N}\right)\right|
$$

No apêndice são apresentadas as figuras A.1, A.2-A.6 dos boxplots para MXDV obtidos com as simulações feitas ao aplicar a fórmula (2.28). Nos gráficos obtidos, V1 representa os valores de MXDV com ondaletas deformadas para a base Symmlet8; V2 representa os valores de MXDV obtidos ao aplicar as ondaletas deformadas com base coiflet3.

De modo geral, pode-se observar que, para cada uma das respectivas bases de ondaletas estudadas, as estimativas encontradas com um $r s n=1$ apresentam valores maiores no MXDV do que as obtidas para o valor de $r s n r=7$. Com respeito às bases de ondaleta, observa-se que os valores de MXDV são similares para cada um dos níveis de ruído analisados, exceto no caso em que $f(x)=$ Doppler e $g(x)=$ constante (com $r s n r=7$ e para $N=512$ ) quando os valores de MXDV são maiores para a ondaleta Coiflet3.

\subsection{Provas}

\subsubsection{Proposição 2.3.1}

(a) Este item é provado em Kerkyacharian e Picard (2004). 
(b) Sob as suposições 1 a 6 , para o estimador (2.6) temos

$$
\begin{aligned}
& \operatorname{cov}\left[\hat{\beta}_{j, k} ; \hat{\beta}_{j^{\prime}, k^{\prime}}\right]=\operatorname{cov}\left[N^{-1} \sum_{t=1}^{N} \psi_{j, k}\left(G\left(X_{t}\right)\right) Y_{t} ; N^{-1} \sum_{s=1}^{N} \psi_{j^{\prime}, k^{\prime}}\left(G\left(X_{s}\right)\right) Y_{s}\right] \\
& =N^{-2} \sum_{t=1}^{N} \sum_{s=1}^{N}\left\{\operatorname{cov}\left(\psi_{j, k}\left(G\left(X_{t}\right)\right) f\left(X_{t}\right) ; \psi_{j^{\prime}, k^{\prime}}\left(G\left(X_{s}\right)\right) f\left(X_{s}\right)\right)\right. \\
& +\operatorname{cov}\left(\psi_{j, k}\left(G\left(X_{t}\right)\right) \epsilon_{t} ; \psi_{j^{\prime}, k^{\prime}}\left(G\left(X_{s}\right)\right) f\left(X_{s}\right)\right)+\operatorname{cov}\left(\psi_{j, k}\left(G\left(X_{s}\right)\right) \epsilon_{s} ; \psi_{j^{\prime}, k^{\prime}}\left(G\left(X_{t}\right)\right) f\left(X_{t}\right)\right) \\
& \left.+\operatorname{cov}\left(\psi_{j, k}\left(G\left(X_{t}\right)\right) \epsilon_{t} ; \psi_{j^{\prime}, k^{\prime}}\left(G\left(X_{s}\right)\right) \epsilon_{s}\right)\right\} \\
& =A+B+C+D
\end{aligned}
$$

Analisando o termo A, considera-se $W_{t, j, k}=\psi_{j, k}\left(G\left(X_{t}\right)\right) f\left(X_{t}\right)$. Esta é uma variável aleatória em função de $X_{t}$, onde $\left\{X_{t}\right\}$ é uma sequência $\alpha$-mixing, logo $\left\{W_{t}\right\}$ também é uma sequência $\alpha$-mixing.

Vejamos agora as características de $W_{t}$. Pode ser facilmente verificado que $E\left[W_{t}\right]=0$ e

$$
\begin{aligned}
& \operatorname{Cov}\left(W_{t ; j, k} ; W_{s ; j^{\prime} . k^{\prime}}\right)=\operatorname{cov}\left(\psi_{j, k}\left(G\left(X_{t}\right)\right) f\left(X_{t}\right) ; \psi_{j^{\prime}, k^{\prime}}\left(G\left(X_{s}\right)\right) f\left(X_{s}\right)\right) \\
& \left.\leq 8 \alpha_{W_{|t-s|, j, k}}^{1 / r}\left(E\left[\left|\psi_{j, k}\left(G\left(X_{t}\right)\right) f\left(X_{t}\right)\right|^{p}\right]\right)^{1 / p}\left(\left.E\left[\mid \psi_{j^{\prime}, k^{\prime}}\left(G\left(X_{s}\right)\right) f\left(X_{s}\right)\right)\right|^{q}\right]\right)^{1 / q}
\end{aligned}
$$

para $p, q, r \geq 0$ e $1 / p+1 / q+1 / r=1$. Calculando as esperanças na expresão anterior, verifica-se:

$$
\begin{aligned}
& E\left[\left|\psi_{j, k}\left(G\left(X_{t}\right)\right) f\left(X_{t}\right)\right|^{p}\right]=\int_{a}^{b}\left|\psi_{j, k}(G(x)) f(x)\right|^{p} g(x) d x=\int_{0}^{1}\left|\psi_{j, k}(y) f\left(G^{-1}(y)\right)\right|^{p} d y \\
& \leq\|f\|_{\infty}^{p} \int_{0}^{1}\left|\psi_{j, k}(y)\right|^{p} d y \leq\|f\|_{\infty}^{p} 2^{j(p-2) / 2}\|\psi\|_{\infty}^{p-2} \int_{0}^{1}\left|\psi_{j, k}(y)\right|^{2} d y .
\end{aligned}
$$

Logo,

$$
E\left[\left|\psi_{j k}\left(G\left(X_{t}\right)\right) f\left(X_{t}\right)\right|^{p}\right] \leq\|f\|_{\infty}^{p} 2^{j(p-2) / p}\|\psi\|_{\infty}^{p-2}
$$

Do mesmo modo, temos que $E\left[\left|\psi_{j^{\prime} k^{\prime}}\left(G\left(X_{s}\right)\right) f\left(X_{s}\right)\right|^{q}\right] \leq\|f\|_{\infty}^{q} 2^{j^{\prime}(q-2) / q}\|\psi\|_{\infty}^{q-2}$.

Das relações anteriores, podemos concluir:

$$
\operatorname{cov}\left(W_{t ; j . k}, W_{s ; j^{\prime}, k^{\prime}}\right) \leq 8 \alpha_{W_{|s-t|}^{1 / r}}^{1 / f}\|\|_{\infty} 2^{j(p-2) / 2 p+j^{\prime}(q-2) / 2 q}\|\psi\|_{\infty}^{(p-2) / p+(q-2) / q}
$$


Por outo lado, considerando o termo D, observa-se:

$$
\operatorname{cov}\left(\psi_{j, k}\left(G\left(X_{t}\right)\right) \epsilon_{t} ; \psi_{j^{\prime}, k^{\prime}}\left(G\left(X_{s}\right)\right) \epsilon_{s}\right)=E\left[\psi_{j, k}\left(G\left(X_{t}\right)\right) \psi_{j^{\prime}, k^{\prime}}\left(G\left(X_{s}\right)\right)\right] E\left[\epsilon_{t} \epsilon_{s}\right]
$$

$\log 0$

$$
\operatorname{cov}\left[\left(\psi_{j, k}\left(G\left(X_{t}\right)\right) \epsilon_{t} ; \psi_{j, k}\left(G\left(X_{s}\right)\right) \epsilon_{s}\right)\right]= \begin{cases}\sigma_{\epsilon}^{2} \int_{a}^{b} \psi_{j, k}\left(G\left(X_{t}\right)\right) \psi_{j^{\prime}, k^{\prime}}\left(G\left(X_{s}\right)\right) g(x) d x, & \text { se } t=s . \\ 0, & \text { se } s \neq t\end{cases}
$$

Também é possível verificar que B e C são iguais a zero, conforme a expressão abaixo:

$$
\left.\operatorname{cov}\left(\psi_{j k}\left(G\left(X_{t}\right)\right) f\left(X_{t}\right) ; \psi_{j, k}\left(G\left(X_{s}\right)\right) \epsilon_{s}\right)=E\left[\psi_{j k}\left(G\left(X_{t}\right)\right) \psi_{j, k}\left(G(X)_{s}\right) f\left(X_{t}\right)\right] E\left[\epsilon_{s}\right]=\emptyset 2.33\right)
$$

Por simetria, temos: $\operatorname{cov}\left(\psi_{j k}\left(G\left(X_{s}\right)\right) f\left(X_{s}\right) ; \psi_{j, k}\left(G\left(X_{t}\right)\right) \epsilon_{t}\right)=0$. Dos resultados obtidos em (2.30), (2.32) e (2.33) conclui-se:

$$
\begin{aligned}
\operatorname{cov}\left[\hat{\beta}_{j, k} ; \hat{\beta}_{j^{\prime}, k^{\prime}}\right] & \leq N^{-1}\left(8 \alpha_{W_{|s-t|}}^{1 / r}\|f\|_{\infty} 2^{j(p-2) / 2 p+j^{\prime}(q-2) / 2 q}\|\psi\|_{\infty}^{(p-2) / p+(q-2) / q}\right) \\
& +\sigma_{\epsilon}^{2} \int_{a}^{b} \psi_{j, k}\left(G\left(X_{t}\right)\right) \psi_{j^{\prime}, k^{\prime}}\left(G\left(X_{s}\right)\right) g(x) d x \\
& +\sum_{t=1}^{N} \sum_{s \neq t}^{N}\left(8 \alpha_{W_{|s-t|}}^{1 / r}\|f\|_{\infty} 2^{j(p-2) / 2 p+(q-2) / 2 q}\|\psi\|_{\infty}^{(p-2) / p+(q-2) / q}\right)
\end{aligned}
$$

para $p, q, r \geq 1$ e $1 / p+1 / q+1 / r=1$, para $j \neq j^{\prime}$ e $k \neq k^{\prime}$. 
(c)

$$
\operatorname{Var}\left[\hat{\beta}_{j, k}\right]=\operatorname{Var}\left[N^{-1} \sum_{t=1}^{N} \psi_{j, k}\left(G\left(X_{t}\right)\right)\left(f\left(X_{t}\right)+\epsilon_{t}\right)\right]=N^{-2} \operatorname{Var}\left[\sum_{t=1}^{N} \psi_{j, k}\left(G\left(X_{t}\right)\right)\left(f\left(X_{t}\right)+\epsilon_{t}\right)\right]
$$

em que

$$
\begin{aligned}
& \operatorname{Var}\left[\sum_{t=1}^{N} \psi_{j, k}\left(G\left(X_{t}\right)\right)\left(f\left(X_{t}\right)+\epsilon_{t}\right)\right]=\sum_{t=1}^{N} \operatorname{Var}\left[\psi_{j, k}\left(G\left(X_{t}\right)\right)\left(f\left(X_{t}\right)+\epsilon_{t}\right)\right] \\
& +2 \sum_{s>t} \operatorname{cov}\left(\psi_{j, k}\left(G\left(X_{t}\right)\right) \psi_{j, k}\left(G\left(X_{s}\right)\right) f\left(X_{t}\right) f\left(X_{s}\right)\right) \\
& \operatorname{Var}\left[\psi_{j, k}\left(G\left(X_{t}\right)\right)\left(f\left(X_{t}\right)+\epsilon_{t}\right)\right]=\operatorname{Var}\left[\psi_{j, k}\left(G\left(X_{t}\right)\right) f\left(X_{t}\right)\right]+\operatorname{Var}\left[\psi_{j, k}\left(G\left(X_{t}\right)\right) \epsilon_{t}\right] \\
& \operatorname{Var}\left[\psi_{j, k}\left(G\left(X_{t}\right)\right) f\left(X_{t}\right)\right]=E\left[\psi_{j, k}^{2}\left(G\left(X_{t}\right)\right) f^{2}\left(X_{t}\right)\right]-\left(E\left[\psi_{j, k}\left(G\left(X_{t}\right)\right) f\left(X_{t}\right)\right]\right)^{2} \\
& E\left[\psi_{j, k}^{2}\left(G\left(X_{t}\right)\right) f^{2}\left(X_{t}\right)\right]=\int_{a}^{b} \psi_{j, k}^{2}(G(x)) f^{2}(x) g(x) d x=\int_{0}^{1} \psi_{j, k}^{2}(y) f^{2}\left(G^{-1}(y)\right) d y \\
& \leq\|f\|_{\infty}^{2} \int_{0}^{1} \psi_{j, k}^{2}(y) d y \leq\|f\|_{\infty}^{2} \\
& \operatorname{Var}\left[\psi_{j, k}\left(G\left(X_{t}\right)\right) \epsilon_{t}\right]=E\left[\psi_{j, k}^{2}\left(G\left(X_{t}\right)\right) \epsilon_{t}^{2}\right]=E\left[\psi_{j, k}^{2}(G(X))\right] E\left[\epsilon_{t}^{2}\right]=\sigma_{\epsilon}^{2} .
\end{aligned}
$$

Logo:

$$
\operatorname{Var}\left[\psi_{j, k}\left(G\left(X_{t}\right)\right)\left(f\left(X_{t}\right)+\epsilon_{t}\right)\right] \leq\left(\|f\|_{\infty}^{2}-\beta_{j, k}^{2}\right)+\sigma_{\epsilon}^{2}
$$

De (2.30) e (2.35) segue que:

$\operatorname{Var}\left[\hat{\beta}_{j, k}\right] \leq N^{-1}\left(\|f\|_{\infty}^{2}+\sigma_{\epsilon}^{2}-\beta_{j, k}^{2}\right)+N^{-1}\left(8 \alpha_{W_{|s-t|}}^{1 / r}\|f\|_{\infty} 2^{j(p-2) / 2 p+j(q-2) / 2 q}\|\psi\|_{\infty}^{(p-2) / p+(q-2) / q}(2.36)\right.$

$\operatorname{com} p, q, r \geq 1,1 / p+1 / q+1 / r=1$.

\subsubsection{Teorema 2.3.1}

$$
E\|\hat{f}-f\|_{p}^{p} \leq 2^{(p-1)}\left[E\left\|\sum_{(j, k) \in \mathcal{J}_{N}}\left(\hat{\beta}_{j k} \mathrm{I}_{\left|\hat{\beta}_{j k}\right|>\frac{K t_{N}}{2}}-\beta_{j k}\right) \psi_{j k}(G(x))\right\|_{p}^{p}+\left\|\sum_{(j, k) \notin \mathcal{J}_{N}} \beta_{j k} \psi_{j k}(G(x))\right\|_{p}^{p}\right]_{2.37)}
$$


Seja

$$
\begin{aligned}
& A^{*}=E\left\|\sum_{(j, k) \in \mathcal{J}_{N}}\left(\hat{\beta}_{j k} \mathrm{I}_{\left|\hat{\beta}_{j k}\right|>\frac{\mathcal{K} t_{N}}{2}}-\beta_{j k}\right) \psi_{j k}(G(x))\right\|_{p}^{p} \\
& =2^{p-1} E\left\|\sum_{(j, k) \in \mathcal{J}_{N}}\left(\hat{\beta}_{j k}-\beta_{j k}\right) \mathrm{I}_{\left|\hat{\beta}_{j k}\right|>\frac{\mathcal{K} t_{N}}{2}}+\sum_{(j, k) \in \mathcal{J}_{N}} \mathrm{I}_{\left|\hat{\beta}_{j k}\right|<\frac{\mathcal{K} t_{N}}{2}} \beta_{j k} \psi_{j k}(G(x))\right\|_{p}^{p} \\
& \left.\leq 2^{(p-1)} E\left\|\sum_{(j, k) \in \mathcal{J}_{N}}\left(\hat{\beta}_{j k}-\beta_{j k}\right) \mathrm{I}_{\left|\hat{\beta}_{j k}\right|>\frac{\Re t_{N}}{2}}\right\|_{p}^{p}+E\left\|\sum_{(j, k) \in \mathcal{J}_{N}} \mathrm{I}_{\left|\hat{\beta}_{j k}\right|<\frac{\kappa t_{N}}{2}} \beta_{j k} \psi_{j k}(G(x)) 2\right\|_{p}^{p} 38\right) \\
& =A+B \text {. }
\end{aligned}
$$

Aplicando a propriedade (1.30) em A obtém-se

$$
\begin{aligned}
& A=2^{(p-1)} K\left\{E \int\left(\sum_{(j, k) \in \mathcal{J}_{N}} \mathrm{I}_{\left|\beta_{j k}\right| \leq \mathcal{K} t_{N} / 2}\left|\hat{\beta}_{j k}-\beta_{j k}\right|^{2} \mathrm{I}_{\left|\hat{\beta}_{j k}-\beta_{j k}\right|>\mathcal{K} t_{N} / 2}\left|\psi_{j k}\right|^{2}\right)^{p / 2}\right. \\
& \left.+E \int\left(\sum_{(j, k) \in \mathcal{J}_{N}} \mathrm{I}_{\left|\beta_{j k}\right|>\mathcal{K} t_{N} / 2}\left|\hat{\beta}_{j k}-\beta_{j k}\right|^{2} \mathrm{I}_{\left|\hat{\beta}_{j k}\right|>\mathcal{K} t_{N}} \psi_{j k}(G(x))\right)^{p / 2}\right\} \\
& =A_{1}+A_{2} .
\end{aligned}
$$

Aplicando a propriedade (1.30) a $A_{1}$

$$
A_{1} \leq \int\left(\sum_{(j, k) \in \mathcal{J}_{N}} E\left[\mathrm{I}_{\left|\beta_{j k}\right| \leq \mathcal{K} t_{N} / 2}\left|\hat{\beta}_{j k}-\beta_{j k}\right|^{2} \mathrm{I}_{\left|\hat{\beta}_{j k}-\beta_{j k}\right|>\mathcal{K} t_{N} / 2}\left|\psi_{j k}\right|^{2}\right]\right)^{p / 2}
$$

Por outra lado, aplicando a desigualdade de Schwartz tem-se que

$$
\begin{aligned}
& E\left[\left|\hat{\beta}_{j k}-\beta_{j k}\right|^{p} \mathrm{I}_{\left|\hat{\beta}_{j k}-\beta_{j k}\right|>\mathcal{K} t_{N} / 2}\left|\psi_{j k}\right|^{p}\right] \\
& \leq\left(P\left(\left|\hat{\beta}_{j k}-\beta_{j k}\right|>\mathcal{K} t_{N / 2}\right)\right)^{1 / 2}\left(E\left[\left|\hat{\beta}_{j k}-\beta_{j k}\right|^{2 p}\right]\right)^{1 / 2}\left|\psi_{j k}\right|^{p} \\
& \leq\left(C_{p} N^{\gamma p / 2}\right)\left(C t_{N}^{4 p}\right)^{1 / 2}\left|\psi_{j k}\right|^{p}=C_{p} C^{*} N^{\gamma p / 2} t_{N}^{2 p}\left|\psi_{j k}\right|^{p} .
\end{aligned}
$$


Para $p \leq 2$

$$
\begin{aligned}
A_{1} & \leq E \int\left[\sum_{(j, k) \in \mathcal{J}_{N},\left|\beta_{j k}\right| \leq \mathcal{K} t_{N} / 2}\left|\hat{\beta}_{j k}-\beta_{j k}\right|^{2} \mathrm{I}_{\left|\hat{\beta}_{j k}-\beta_{j k}\right|>\mathcal{K} t_{N} / 2}\left|\psi_{i j}\right|^{2}\right]^{p / 2} \\
& \leq \int E\left[\sum_{(j, k) \in \mathcal{J}_{N},\left|\beta_{j k}\right| \leq \mathcal{K} t_{N} / 2}\left|\hat{\beta}_{j k}-\beta_{j k}\right|^{p} \mathrm{I}_{\left|\hat{\beta}_{j k}-\beta_{j k}\right|>\mathcal{K} t_{N} / 2}\left|\psi_{i j}(G(x))\right|^{p}\right] \\
& \leq C_{p} C^{*} N^{-\gamma p / 2} t_{N}^{2 p} \int_{(j, k) \in \mathcal{J}_{N},\left|\beta_{j k}\right| \leq \mathcal{K} t_{N} / 2}\left|\psi_{i j}(G(x))\right|^{p} \\
& \leq C_{p} t_{N}^{2 p} C^{*} N^{-\gamma p / 2} \nu\left\{(j, k) \in \mathcal{J}_{N},\left|\beta_{j k}\right| \leq \mathcal{K} t_{N} / 2\right\}
\end{aligned}
$$

Na linha 2 foi usada a propriedade (1.30) e na linha tres foi usada a desigualdade (2.42).

A seguir é estudado o caso para $p \geq 2$,

$$
\begin{aligned}
A_{1} & \leq \int\left\{\sum_{(j, k) \in \mathcal{J}_{N},\left|\beta_{j k}\right| \leq \mathcal{K} t_{N} / 2}\left[E\left|\hat{\beta}_{j k}-\beta_{j k}\right|^{p} \mathrm{I}_{\left|\hat{\beta}_{j k}-\beta_{j k}\right|>\mathcal{K} t_{N} / 2}\left|\psi_{j k}(G(x))\right|^{p}\right]^{2 / p}\right\}^{p / 2} \\
& \leq C t_{N}^{2 p} N^{-\gamma p / 2} \int\left\{\sum_{(j, k) \in \mathcal{J}_{N},\left|\beta_{j k}\right| \leq \mathcal{K} t_{N} / 2}\left|\psi_{j k}(G(x))\right|^{2}\right\}^{p / 2}\left|\psi_{j k}(G(x))\right|^{p} \\
& \leq C t_{N}^{2 p} N^{-\gamma p / 2} \int \sum_{(j, k) \in \mathcal{J}_{N},\left|\beta_{j k}\right| \leq \mathcal{K} t_{N} / 2} \sum_{\left\{(j, k) \in \mathcal{J}_{N},\left|\beta_{j k}\right| \leq \mathcal{K} t_{N} / 2\right\}}
\end{aligned}
$$

Primeiro é usada a desigualdade generalizada de Minkowski, na segunda linha é usada a desigualdade (2.42) e logo é utilizada a propriedade (1.30).

Para $A_{2}$, o procedimento é análogo ao feito com $A_{1}$, logo, para $p \leq 2$, se tem:

$$
A_{2} \leq C t_{N}^{p} N^{-\gamma^{*} p / 2} \nu\left\{(j, k) \in \mathcal{J}_{N},\left|\beta_{j k}\right|>\mathcal{K} t_{N} / 2\right\}
$$

e para $p \geq 2$

$$
A_{2} \leq C t_{N}^{p} N^{\gamma^{*} p / 2} \nu\left\{(j, k) \in \mathcal{J}_{N},\left|\beta_{j k}\right|>\mathcal{K} t_{N} / 2\right\}
$$


Agora vamos analizar o termo B

$$
\begin{aligned}
B & =E \| \sum_{(j, k) \in \mathcal{J}_{N}} \mathrm{I}_{\left|\hat{\beta}_{j k}\right|<\frac{\mathcal{K} t_{N}}{2} \beta_{j k} \psi_{j k}(G(x)) \|_{p}^{p}} \\
& =2^{(p-1)} K\left[E \int\left(\sum_{(j, k) \in \mathcal{J}_{N},\left|\beta_{j k}\right|>2 \mathcal{K} t_{N}}\left|\beta_{j k}\right|^{2} \mathrm{I}_{\left|\hat{\beta}_{j k}-\beta_{j k}\right|>\mathcal{K} t_{N}}\left|\psi_{j k}(G(x))\right|^{2}\right)^{p / 2}\right. \\
& \left.+E \int\left(\sum_{(j, k) \in \mathcal{J}_{N},\left|\beta_{j k}\right| \leq 2 \mathcal{K} t_{N}}\left|\beta_{j k}\right|^{2} \mathrm{I}_{\left|\hat{\beta}_{j k}\right| \leq \mathcal{K} t_{N}}\left|\psi_{j k}(G(x))\right|^{2}\right)^{p / 2}\right] \\
& =B_{1}+B_{2}
\end{aligned}
$$

Para $p \leq 2$

$$
\begin{aligned}
B_{1} & \leq \int\left\{E \sum_{(j, k) \in \mathcal{J}_{N},\left|\beta_{j k}\right|>2 \mathcal{K} t_{N}}\left|\beta_{j k} \psi_{j k}(G(x))\right|^{2} \mathrm{I}_{\left|\hat{\beta}_{j k}-\beta_{j k}\right| \geq \mathcal{K} t_{N}}\right\}^{p / 2} \\
& \leq C t_{N}^{2 p} \int\left\{\sum_{(j, k) \in \mathcal{J}_{N},\left|\beta_{j k}\right|>2 \mathcal{K} t_{N}}\left|\beta_{j k} \psi_{j k}(G(x))\right|^{2}\right\}^{p / 2} \\
& \leq C t_{N}^{2 p}\|f\|_{p}^{p} .
\end{aligned}
$$

Na primeira linha é usada a desigualdade de Jensen, na linha dois é usada a desigualdade (2.42) e logo é aplicada a propriedade (1.29).

Para $p \geq 2$, é usado a desigualdade generalizada de Minkowski

$$
\begin{aligned}
B_{1} & \leq \int\left\{\sum_{(j, k) \in \mathcal{J}_{N},\left|\beta_{j k}\right|>2 \mathcal{K} t_{N}}\left[E\left|\beta_{j k}\right|^{p} \mathrm{I}_{\left|\hat{\beta}_{j k}-\beta_{j k}\right| \geq \mathcal{K} t_{N}}\left|\psi_{j k}(G(x))\right|^{p}\right]^{2 / p}\right\}^{p / 2} \\
& \leq C t_{N}^{2 p} \int\left\{\sum_{(j, k) \in \mathcal{J}_{N},\left|\beta_{j k}\right|>2 \mathcal{K} t_{N}}\left|\beta_{j k} \psi_{j k}(G(x))\right|^{2}\right\}^{p / 2}\left|\beta_{j k} \psi_{j k}(G(x))\right|^{p} \\
& \leq C t_{N}^{2 p} \int \sum_{(j, k) \in \mathcal{J}_{N},\left|\beta_{j k}\right|>2 \mathcal{K} t_{N}} \\
& \leq C t_{N}^{2 p}\|f\|_{p}^{p} .
\end{aligned}
$$


Por outro lado,

$$
\begin{aligned}
& B_{2} \leq \int\left\{\sum_{(j, k) \in \mathcal{J}_{N},\left|\beta_{j k}\right| \leq 2 \mathcal{K} t_{N}}\left|\beta_{j k}\right|^{2}\left|\psi_{j k}(G(x))\right|^{2}\right\}^{p / 2} \\
& \leq K^{p}\left\|\sum_{(j, k) \in \mathcal{J}_{N},\left|\beta_{j k}\right| \leq 2 \mathcal{K} t_{N}} \beta_{j k} \psi_{j k}\right\|_{p}^{p} \\
& \leq K^{p}\left[\sum_{K \geq 0}\left\|\sum_{(j, k) \in \mathcal{J}_{N}, 2^{-k} 2 \mathcal{K} t_{N} \leq\left|\beta_{j k}\right| \leq 2^{-k+1} \mathcal{K} t_{N}} \beta_{j k} \psi_{j k}\right\|_{p}\right]^{p} \\
& \leq K^{p}\left[\sum_{k \geq 0} 2^{-k+1} \mathcal{K} t_{N}\left\|\sum_{(j, k) \in \mathcal{J}_{N}, 2^{-k} 2 \mathcal{K} t_{N} \leq\left|\beta_{j k}\right| \leq 2^{-k+1} \mathcal{K} t_{N}} \psi_{j k}(G(x))\right\|_{p}\right]^{p} \\
& \leq K^{p}\left[\sum_{k \geq 0} 2^{-k+1} \mathcal{K} t_{N}\left\{\sum_{(j, k) \in \mathcal{J}_{N}, 2^{-k} 2 \mathcal{K} t_{N} \leq\left|\beta_{j k}\right| \leq 2^{-k+1} \mathcal{K} t_{N}}\left\|\psi_{j k}(G(x))\right\|_{p}\right\}^{1 / p}\right]^{p} \\
& \leq K^{p}\left[\sum_{K \geq 0} 2^{-k+1} \mathcal{K} t_{N}\left\{2^{-k} \mathcal{K} t_{N}\right\}^{-q / p}\|f\|_{l_{q, \infty}}^{q / p}\right]^{p} \\
& \leq C t_{N}^{p-q}\|f\|_{l_{q, \infty}}^{q} \text {. }
\end{aligned}
$$

Na linha 1 é usado a propriedade (1.29), na linha três é aplicada a desigualdade triangular, na linha 4 de novo a propriedade (1.29), na linha 5 é usada a propriedade (1.30).

Resumindo se tem:

$$
\begin{aligned}
E\|\hat{f}-f\|_{p}^{p} & \leq C_{p} t_{N}^{2 p} C^{*} N^{-\gamma p / 2} \nu\left\{(j, k) \in \mathcal{J}_{N},\left|\beta_{j k}\right| \leq \mathcal{K} t_{N} / 2\right\}+C t_{N}^{p} N^{\gamma^{*} p / 2} \nu\left\{(j, k) \in \mathcal{J}_{N},\left|\beta_{j k}\right|>\mathcal{K} t_{N} / 2\right\} \\
& +C t_{N}^{2 p}\|f\|_{p}^{p}+C t_{N}^{p-q}\|f\|_{l_{q, \infty}}^{q}+\left\|\sum_{(j, k) \notin \mathcal{J}_{N}} \beta_{j k} \psi_{j k}(G(x))\right\|_{p}^{p} \\
& \leq t_{N}^{p-q} A\left(\|f\|_{l_{q, \infty}}^{q}+t_{N}^{p+q}\left[\|f\|_{p}^{p}+\nu\left\{(j, k) \in \mathcal{J}_{N}\right\}\right]\right)+\left\|\sum_{(j, k) \notin \mathcal{J}_{N}} \beta_{j k} \psi_{j k}(G(x))\right\|_{p}^{p},
\end{aligned}
$$

onde A é uma constante que depende de p,q,k e $C_{p}$. Expressando $p-q$ em termos de $s$, se tem

$$
t_{N}^{2 s p /(1+2 s)} A\left(\|f\|_{l_{q, \infty}}^{q}+t_{N}^{p+q}\left[\|f\|_{p}^{p}+\nu\left\{(j, k) \in \mathcal{J}_{N}\right\}\right]\right)+\left\|\sum_{(j, k) \notin \mathcal{J}_{N}} \beta_{j k} \psi_{j k}(G(x))\right\|_{p}^{p}
$$




\subsubsection{Proposição 2.3.2}

(a) De forma geral, seguimos os mesmos passos da prova dados em Porto (2008).

$$
E\left[\left|\hat{\beta}_{j, k}-\beta_{j, k}\right|^{m}\right]=N^{-m} E\left[\left|\sum_{t=1}^{N} \psi_{j, k}\left(G\left(X_{t}\right)\right) Y_{t}-\beta_{j, k}\right|^{m}\right]
$$

Seja $Z_{t ; j, k}=\psi_{j, k}\left(G\left(X_{t}\right)\right) Y_{t}-\beta_{j, k}$. Portanto, $E\left[\left|\hat{\beta}_{j, k}-\beta_{j, k}\right|^{m}\right]=N^{-m} E\left[\left|Z_{t ; j, k}\right|^{m}\right]$. Por outro lado, temos que $E\left[Z_{t ; j, k}\right]=0$.

$$
\begin{aligned}
& \operatorname{Cov}\left(Z_{t ; j, k}, Z_{s ; j, k}\right)=\operatorname{Cov}\left(\psi_{j, k}\left(G\left(X_{t}\right)\right) Y_{t}-\beta_{j, k} ; \psi_{j, k}\left(G\left(X_{s}\right)\right) Y_{s}-\beta_{j, k}\right) \\
& =\operatorname{Cov}\left(\psi_{j, k}\left(G\left(X_{t}\right)\right) Y_{t}, \psi_{j, k}\left(G\left(X_{s}\right)\right) Y_{s}\right)+\operatorname{Cov}\left(\psi_{j, k}\left(G\left(X_{t}\right)\right) Y_{t}, \beta_{j, k}\right) \\
& \left.=\operatorname{Cov}\left(\psi_{j, k}\left(G\left(X_{t}\right)\right) f\left(X_{t}\right), \psi_{j, k}\left(G\left(X_{s}\right)\right) f\left(X_{s}\right)\right)+\operatorname{Cov}\left(\psi_{j, k}\left(G\left(X_{t}\right)\right) \epsilon_{t}, \psi_{j, k}\left(G\left(X_{s}\right)\right) \epsilon_{s}\right)\right),
\end{aligned}
$$

Considerando as expressões (2.32) e (2.30), observa-se:

$$
\begin{aligned}
& \operatorname{Cov}\left(Z_{t ; j, k}, Z_{s ; j, k}\right)=N\left(8 \alpha_{W_{|s-t|}^{1 / r}}^{1 / r}\|f\|_{\infty} 2^{j(p-2) / 2 p+(q-2) / 2 q}\|\psi\|_{\infty}^{(p-2) / p+(q-2) / q}\right) \\
& +N^{-1} \sigma^{2} \int_{a}^{b} \psi_{j, k}(G(X)) \psi_{j^{\prime}, k^{\prime}}(G(X)) g(x) d x \\
& +\sum_{t=1}^{N} \sum_{s \neq t}^{N}\left(8 \alpha_{W_{|s-t|}^{1 / r}}^{1 / r}\|f\|_{\infty} 2^{j(p-2) / 2 p+(q-2) / 2 q}\|\psi\|_{\infty}^{(p-2) / p+(q-2) / q}\right)
\end{aligned}
$$

Pela suposição 6 , o coeficiente mixing $\alpha_{X, h}=0$; de forma similar definimos o coeficiente mixing $\alpha_{z_{j, k}, h}$. Como este coeficiente mixing é definido nas $\sigma$-álgebras, então ele apenas pode ser menor ou igual a $\alpha_{X, h}$, o qual por sua vez satisfaz a suposição 7 . A mesma suposição vale para $\alpha_{z_{j, k}, h}$. Portanto, aplicando o Teorema 2, conforme expresso em Doukhan (1994) pag 26, para $m>2$, temos:

$\left.N^{-m} E\left(\left|\sum_{t=1}^{N} Z_{t ; j, k}\right|^{m}\right) \leq \frac{C}{N^{m}} \max \left\{\sum_{t=1}^{N}\left[E\left(\left|Z_{t ; j, k}\right|^{m+\delta}\right)\right]^{\frac{m}{m+\delta}},\left[\sum_{t=1}^{N}\left(E\left(\left|Z_{t ; j, k}\right|^{2+\delta}\right)\right)^{\frac{2}{2+\delta}}\right]^{p / 2}\right\} 2.56\right)$

Todos os passos seguintes são iguais a Porto (2008), logo:

$$
E\left|\hat{\beta}_{j, k}-\beta_{j, k}\right|^{m} \leq C(\log N / N)^{m / 2}
$$


para todo $2^{J_{1}} \leq \operatorname{Cmin}\left\{N_{1}, N_{2}\right\}$, onde

$$
N_{1}=\left(\frac{N}{\log N}\right)^{1 / 2}, \quad N_{2}=\left(\frac{N^{(3 m-2) / m}}{\log N}\right)^{\frac{m+\delta}{p+\delta-2}} .
$$

(b) Os passos principais desta prova são os mesmos de Kerkyacharian e Picard (2004). Entretanto, temos que considerar que, como os dados são correlacionados, algumas modificações deverão ser feitas.

$$
\begin{aligned}
& \hat{\beta}_{j, k}-\beta_{j, k}=N^{-1} \sum_{j=1}^{N} \psi_{j, k}\left(G\left(X_{t}\right)\right)\left(f\left(X_{t}\right)+\epsilon_{t}\right)-\beta_{j, k} \\
& =N^{-1} \sum_{t=1}^{N} \psi_{j, k}\left(G\left(X_{t}\right)\right) f\left(X_{t}\right)-E\left[\psi_{j, k}\left(G\left(X_{t}\right)\right) f\left(X_{t}\right)\right]+N^{-1} \sum_{t=1}^{N} \psi_{j, k}\left(G\left(X_{t}\right)\right) \epsilon_{t}
\end{aligned}
$$

Inicialmente, observemos que dado $X_{1}=x_{1}, \ldots, X_{N}=x_{N}$,

$$
N^{-1} \sum_{t=1}^{N} \psi_{j, k}\left(G\left(X_{t}\right)\right) \epsilon_{t}
$$

tem uma distribuição normal com média zero e variância dada por:

$$
\begin{aligned}
& \operatorname{Var}\left[N^{-1} \sum_{t=1}^{N} \psi_{j, k}\left(G\left(X_{t}\right)\right) \epsilon_{t}\right]=E\left[N^{-1} \sum_{t=1}^{N} \psi_{j, k}\left(G\left(X_{t}\right)\right) \epsilon_{t}\right]^{2} \\
& =N^{-2} \sum_{t=1}^{N} E\left[\psi_{j, k}^{2}\left(G\left(X_{t}\right)\right) \epsilon_{t}^{2}\right]=N^{-2} \sum_{t=1}^{N} \psi_{j, k}^{2}\left(G\left(X_{t}\right) E\left[\epsilon_{t}^{2}\right]=N^{-2} \sigma_{\epsilon}^{2} \sum_{t=1}^{n} \psi_{j, k}^{2}\left(G\left(X_{t}\right)\right) .\right.
\end{aligned}
$$

Sabemos que $\left\|\psi_{j, k}^{2}\left(G\left(X_{t}\right)\right)\right\| \leq 2^{j}\|\psi\|_{\infty}^{2}, \operatorname{logo}$

$$
\operatorname{Var}\left[N^{-1} \sum_{t=1}^{N} \psi_{j, k}\left(G\left(X_{t}\right)\right) \epsilon_{t}\right] \leq N^{-1} 2^{j}\|\psi\|_{\infty}^{2} \sigma_{\epsilon}^{2}
$$

Logo, seguindo as indicações de Kerkyacharian e Picard (2004), temos que:

$$
\begin{aligned}
& P\left(\left|N^{-1} \sum_{t=1}^{N} \psi_{j, k}\left(G\left(X_{t}\right)\right) \epsilon_{t}\right|>\frac{\mathcal{K}}{2} \sqrt{\frac{\log N}{N}}\right) \leq P\left(\left|\sum_{t=1}^{N} \psi_{j, k}^{2}\left(G\left(X_{t}\right)\right)-1\right|>\alpha\right)+ \\
& \exp \left(-\frac{k^{2} \log N}{8(1+\alpha)}\right)
\end{aligned}
$$

e como a sequência $\left\{X_{t}\right\}$ é $\alpha$-mixing, então precisamos utilizar a desigualdade (1.57), 
ressaltando que são satisfeitas as condições (1.54) e (1.55). Pelo Lema 1.6.1, temos que $C_{r, q} \leq 4 M^{q} \alpha_{r}$ portanto (1.54) é satisfeita, com $\epsilon_{r}=\alpha_{r}$.

Agora vamos ver que a condição (1.55) também é satisfeita.

$$
\begin{aligned}
& M_{m, r}=N \sum_{r=0}^{N-1}(r+1)^{m-2} C_{m, r} \leq N \sum_{r=0}^{N-1}(r+1)^{m-2} 4 M^{m} \alpha_{r} \leq 4 N M^{m} \sum_{r=0}^{N-1}(r+1)^{m-2} \alpha_{r} \\
& \leq 4 N M^{m} \sum_{r=0}^{\infty}(r+1)^{m-2} \alpha_{r}^{\delta /(\delta+m)}
\end{aligned}
$$

e pela suposição 7 temos que $\sum_{r=0}^{\infty}(r+1)^{m-2} \alpha_{r}^{\delta /(\delta+m)}<L<\infty$. Logo:

$$
M_{m, r}=N \sum_{r=0}^{N-1}(r+1)^{m-2} C_{m, r} \leq 4 N M^{m} L \leq m ! N M^{m} L, \quad \text { para } m>2 .
$$

Em particular, temos $A_{N}=N L, \beta=1 / M=1 /\left(2^{j}\|\psi\|_{\infty}^{2}\right)$ e $x=N$, portanto

$$
\begin{array}{r}
P\left(\left|\sum_{t=1}^{N} \psi_{j, k}^{2}\left(G\left(X_{t}\right)\right)-1\right|>\alpha\right)=P\left(\left|\sum_{t=1}^{N} \psi_{j, k}^{2}\left(G\left(X_{t}\right)\right)-1\right|>N^{3 / 2} \sqrt{L}\right) \\
\leq A \exp \left\{-B \sqrt{\frac{N}{2^{j}\|\psi\|_{\infty}^{2}}}\right\} \leq A \exp \left\{-B \sqrt{\frac{N \log N}{N\|\psi\|_{\infty}^{2}}}\right\} \\
\leq A \exp \left\{-B \sqrt{\frac{\log N}{\|\psi\|_{\infty}^{2}}}\right\} \leq A \exp \left\{-B \frac{(\log N)^{1 / 2}}{\|\psi\|_{\infty}}\right\} \leq A N^{-\frac{B}{2\|\psi\|_{\infty}}}
\end{array}
$$

$\operatorname{para} 2^{j} \leq\left(\frac{N}{\log N}\right)^{1 / 2}$

Agora vejamos a outra desigualdade:

$$
P\left\{\left|N^{-1} \sum_{t=1}^{N} \psi_{j, k}\left(G\left(X_{t}\right)\right) f\left(X_{t}\right)-E\left[\psi_{j, k}\left(G\left(X_{t}\right)\right) f\left(X_{t}\right)\right]\right|>\mathcal{K} \sqrt{\frac{\log N}{N}}\right\} .
$$

Seja $Q_{t ; j, k}=N^{-1} \sum_{t=1}^{N} \psi_{j, k}\left(G\left(X_{t}\right)\right) f\left(X_{t}\right)-E\left[\psi_{j, k}\left(G\left(X_{t}\right)\right) f\left(X_{t}\right)\right]$, a qual é uma variável $\alpha$-mixing, com $E\left[Q_{t ; j, k}\right]=0$, então podemos utilizar o Corolário (1.6.1). Isso porque, de modo análogo ao que foi provado com a variável $Z_{t ; j, k}$, se tem que a variável $Q_{t ; j, k}$ satisfaz 
as condições $(1.54)$ e $(1.55) \operatorname{com} \beta=1 / M$ e $x=(\log N)^{1 / 2} / N$. Logo temos:

$$
\begin{array}{r}
P\left(N^{-1}\left|\sum_{t=1}^{N} Q_{t ; j, k}\right|>\mathcal{K} \sqrt{\frac{\log N}{N}}\right) \leq A \exp \left\{-B \sqrt{\frac{(\log N)^{1 / 2}}{N^{1 / 2} 2^{j}\|\psi\|_{\infty}^{2}}}\right\} \\
\leq A \exp \left\{-B \sqrt{\frac{(\log N)^{3 / 2}}{N^{3 / 2}\|\psi\|_{\infty}^{2}}}\right\} \leq A \exp \left\{\log N \frac{-B}{4 N^{3 / 2}\|\psi\|_{\infty}}\right\} \\
\leq A N^{-\frac{B}{4 N^{3 / 2}\|\psi\|_{\infty}}} \leq A N^{-\vartheta}
\end{array}
$$

para $2^{j / 2} \leq\left(\frac{N}{\log N}\right)^{1 / 2}$ 



\section{Capítulo 3}

\section{Modelo de Regressão com Variáveis}

\section{Fortemente Mixing Usando Ondaletas Adaptativas de}

\section{Haar}

\subsection{Introdução}

Nesta seção apresentam-se alguns resultados de regressão não paramétrica usando o método de ondaletas adaptativas de Haar, quando a variável explicativa segue um processo $\alpha$-mixing. Um novo limiar que leva em conta a estrutura de dependência dos dados é calculado e as taxas de convergência para obter um risco baixo são obtidas.

\subsection{Procedimento usando Ondaletas Adaptativas de Haar}

Sejam $\left(X_{1}, Y_{1}\right), \ldots,\left(X_{N}, Y_{N}\right)$ pares de variáveis aleatorias observadas. Consideremos o modelo de regressão:

$$
Y_{t}=f\left(X_{t}\right)+\epsilon_{t}, \quad t=1,2, \ldots, N, \quad N=2^{J}, \quad J \in \mathbb{N},
$$

onde $X_{t}$ é uma variável aleatória com função de distribuição $\mathrm{G}, f$ em $\Lambda^{\beta}(L, B, 0)$ é a função a ser estimada com baixo risco e $\epsilon_{t}$ é um termo de erro i.i.d, com $\sigma_{\epsilon}<\infty$. As variáveis $X_{t}$ são independentes de $\epsilon_{t}$, para todo $t$. Suposições sobre $X_{t}$ serão feitas a seguir.

1. A função de densidade $g$ é contínua e positiva no interior de um intervalo compacto $I: g(x) \geq c>0$, para todo $x \in I ; g(x)=0$ para todo $x \in \mathbb{R}-I ;$

2. a função $f$ é Hölder contínua em $I$, com expoente $1 / 2<\beta<1$, isto é, $f \in \Lambda^{\beta}(L, B, 0)$; 
3. a função $f$ é de variação total limitada sob o intervalo $I$;

4. $\left|X_{t}\right| \leq K, \forall t \operatorname{com} K>0$;

5. $X_{t}$ é uma sequência de variáveis aleatórias estacionárias com média zero, variância $\sigma^{2}<\infty$ e $\operatorname{cov}\left(X_{t}, X_{r}\right)=\gamma_{t, r}=\gamma|t-r|$, para todo $r \neq t, r, t \in \mathbb{Z}$ e $\gamma>0$

6. os erros $\left\{\epsilon_{t}\right\}_{t \in \mathbb{Z}}$ são variáveis aleatórias independentes e identicamente distribuídas (i.i.d.), com $E\left[\epsilon_{t}\right]=0, E\left[\epsilon_{t}^{2}\right]=\sigma_{\epsilon}^{2}<\infty$. E $\epsilon_{t}$ é independente de $X_{s}$, para todo $t, s \in \mathbb{Z}$;

7. $\left\{X_{t}\right\}_{t=1}^{N}$ é um processo $\alpha$-mixing com coeficientes $\left.\alpha(s) \leq C e^{\left(-b|s|^{\theta}\right.}\right)$;

8. para todo $M>0$ existe uma constante finita $C_{M}$ tal que $E\left(\left|\epsilon_{t}\right|\right)^{M} \leq C_{M}$.

Da suposição 8 , tem-se que para $\delta>0, \lambda>0$,

$$
P\left(\sup _{t=1, \ldots, N}\left|\epsilon_{t}\right|>c_{\lambda} N^{\delta^{\prime}}\right) \leq c N^{-\lambda}
$$

vale para todo $\delta^{\prime} \in(0, \delta)$, onde $c_{\lambda}$ é uma constante que só depende de $\lambda$.

Suponhamos que:

$$
P\left(\Omega_{N}\right)=P\left(\left\{\omega: \sup \left|\epsilon_{t}(\omega)\right| \leq N^{\delta^{\prime}}\right\}\right) \geq 1-O\left(N^{-\lambda}\right)
$$

ou seja, para um $\lambda$ dado, é possível encontrar um $\delta$, de modo que os $\epsilon_{t}$ são limitados com probabilidade $1-O\left(N^{-\lambda}\right)$, para todo $\delta^{\prime} \in(0, \delta)$.

Na definição do $L_{2}$-risco para avaliar a estimativa $\hat{f}(x)$ obtida pelo método de ondaletas, é considerada como referência a projeção $f_{J}(x)$ de $f(x)$ no espaço gerado por $\psi_{J, k}$, com $J$ definido como o nível mais fino que pertence ao conjunto $\mathcal{J}_{N}$, tal que

$$
\mathcal{J}_{N}=\left\{(j, k) \mid 2^{j} \leq C N^{1-\alpha} ; 1 \leq k \leq 2^{j}\right\}, \quad \text { com } 4 \delta<\alpha<1 .
$$

Assim, tem-se que:

$$
f_{J}(x)=\sum_{k} \hat{b}_{j_{0}, k} \phi_{j_{0}, k}(x)+\sum_{(j, k) \in \mathcal{J}_{N}} \hat{\beta}_{j, k} \psi_{j, k}(x),
$$


onde

$$
\begin{gathered}
\hat{b}_{j k}=\frac{1}{N} \sum_{t=1}^{N} f\left(X_{t}\right) \phi_{j_{0} k}\left(X_{t}\right), \\
\hat{\beta}_{j, k}=\frac{1}{N} \sum_{t=1}^{N} f\left(X_{t}\right) \psi_{j k}\left(X_{t}\right) .
\end{gathered}
$$

Dado um delineamento estatístico $X_{1}, \ldots, X_{N}$, deseja-se encontrar um estimador $\hat{f}$ o mais próximo possível, no sentido de IMSE, para a função projetada $f_{J}(x)$, isto uniformemente para todo $f \in \Lambda^{\beta}(L, B, 0)=\mathcal{F}$. Ou seja, queremos $\hat{f}$ que minimize o risco:

$$
\sup _{f \in \mathcal{F}}\left\{E\left\|\hat{f}-f_{J}\right\|_{L_{2}\left(\hat{G}_{N}\right)}^{2}\right\}
$$

A dificuldade teórica no delineamento adaptativo é tratar os intervalos aleatórios usados para definir as ondaletas. Além disso, estamos tratando com dados com algum grau de dependência. Para contornar este problema, seguimos a técnica de Delouille e Sachs (2004) para modelos autoregressivos. A técnica será descrita a seguir.

Dado um intervalo $\mathcal{I}=I_{j, k}$, como definido em (1.33), este é aproximado pela união de intervalos fixos, começando pela partição de quantis teóricos $I_{j, k}^{0}$,

$$
I_{j, k}^{0}=\left[G_{x}^{-1}\left(\frac{k-1}{2^{j}}\right), G_{x}^{-1}\left(\frac{k}{2^{j}}\right)\right), 0<j<j^{*}, 1 \leq k \leq 2^{j}, 2^{j^{*}} \leq N<2^{j^{*}+1} .
$$

A partição $I^{0}$ é formada por intervalos que contêm índices em uma escala fina, que estão contidos no conjunto de índices $\mathcal{J}_{N}$.

Seguindo Rio (1995), os intervalos aleatórios são tratados da seguinte forma. Considera-se a partição fixa $I_{j, k}^{0}$, começando por recobrir as de resolução mais baixa, aproxima-se $\mathcal{I}$ pela união de intervalos que pertencem a $I^{0}$; onde existem índices $j_{1}, \ldots, j_{d}, k_{1} \ldots, k_{d}$, tais que,

$$
I_{j_{1}, k_{1}}^{0} \cup I_{j_{2}, k_{2}}^{0} \ldots \cup I_{j_{d}, k_{d}}^{0} \subseteq \mathcal{I}
$$

onde $j_{1}<j_{2}<\ldots, j_{d}<j^{*} ; d$, o número de intervalos necessários para aproximar $\mathcal{I}$, é uma função do mesmo $\mathcal{I}$. Mas quando $N$ aumenta, o número $d$ também deve aumentar, por isso é usada a notação $d_{N}$, como em Delouille e Sachs (2004). A seguir, são adicionados dois novos 
intervalos para as escalas finas, $I_{j^{*}, l_{1}}$ e $I_{j^{*}, l_{2}}$, onde $\mathcal{I}$ pode aproximar-se da seguinte forma:

$$
\mathcal{I} \subseteq I_{j_{1}, k_{1}}^{0} \cup I_{j_{2}, k_{2}}^{0} \ldots \cup I_{j_{d}, k_{d}}^{0} \cup I_{j^{*}, l_{1}} \cup I_{j^{*}, l_{2}} .
$$

Note que os índices de escalas finas $j^{*}, l_{i}$ não pertencem ao conjunto de índices $\mathcal{J}_{N}$.

\subsection{Resultados Principais}

Primeiro, vamos identificar o limiar adequado para conseguir remover o ruído com uma probabilidade alta. Consideramos abaixo o coeficiente de detalhe $\alpha_{j, k}$ e seu correspondente termo de erro $\rho_{j, k}$. No caso da ondaleta Haar, tem-se:

$$
\rho_{j, k}=\frac{2^{j / 2}}{N}\left\{\sum_{t=1}^{N} \epsilon_{t} 1_{j+1, k}\left(X_{t}\right)-\sum_{t=1}^{N} \epsilon_{t} 1_{j+1, k+1}\left(X_{t}\right)\right\} .
$$

O propósito é encontrar valores de $a_{j+1, k}^{\lambda}$ e $a_{j+1, k+1}^{\lambda}$ tal que

$$
P\left(\frac{2^{j / 2}}{N}\left|\sum_{t=1}^{N} \epsilon_{t} 1_{j+1, k}\left(X_{t}\right)\right|>a_{j+1, k}^{\lambda}\right) \leq c N^{-\lambda}
$$

$\mathrm{e}$

$$
P\left(\frac{2^{j / 2}}{N}\left|\sum_{t=1}^{N} \epsilon_{t} 1_{j+1, k+1}\left(X_{t}\right)\right|>a_{j+1, k+1}^{\lambda}\right) \leq c N^{-\lambda},
$$

onde $\lambda$ é um número suficientemente grande, $c$ é uma constante e

$$
t_{j, k}=a_{j+1, k}^{\lambda}+a_{j+1, k+1}^{\lambda} .
$$

Assim, concluímos que:

$$
\begin{aligned}
P\left(\left|\rho_{j, k}\right|>t_{j, k}\right) & \leq P\left(\frac{2^{j / 2}}{N}\left|\sum_{t=1}^{N} \epsilon_{t} 1_{j+1, k}\left(X_{t}\right)\right|+\frac{2^{j / 2}}{N}\left|\sum_{t=1}^{N} \epsilon_{t} 1_{j+1, k+1}\left(X_{t}\right)\right|>t_{j, k}\right) \\
& \leq P\left(\frac{2^{j / 2}}{N}\left|\sum_{t=1}^{N} \epsilon_{t} 1_{j+1, k}\left(X_{t}\right)\right|>a_{j+1, k}^{\lambda}\right)+P\left(\frac{2^{j / 2}}{N}\left|\sum_{t=1}^{N} \epsilon_{t} 1_{j+1, k+1}\left(X_{t}\right)\right|>a_{j+1, k+1}^{\lambda}\right) \\
& \leq c N^{-\lambda}
\end{aligned}
$$

Repetindo a operação para todo $(j, k) \in \mathcal{J}_{N}$, com t definido de seguinte maneira:

$$
t(N)=\max _{(j, k) \in \mathcal{J}_{N}} t_{j, k}
$$


ao usar a desigualdade de Bonferroni, obtemos

$$
\begin{aligned}
P\left(\max _{(j, k) \in \mathcal{J}_{N}}\left|\rho_{j, k}\right|>t_{j, k}\right) & =P\left(\bigcup_{(j, k)}^{\cup}\left(\left|\rho_{j, k}\right|>t(N)\right)\right) \\
& \leq \sum_{(j, k) \in \mathcal{J}_{n}} P\left(\left|\rho_{j, k}\right|>t(N)\right)=O\left(N^{1-\alpha-\lambda}\right)
\end{aligned}
$$

com $0<\alpha<1, \lambda$ arbitrário.

O teorema que será apresentado a seguir, permite especificar o melhor limiar $t_{j k}$ para remover o ruído para o caso de regressão não linear com variável explicativa $\alpha$-mixing. Para alcançar uma taxa de convergência quase ótima, os limiares devem ser aplicados aos coeficientes com $(j, k) \in \mathcal{J}_{N}$.

Teorema 3.3.1 Sob as suposições $1-8$, considere o coeficiente de detalhe Haar dado por $\hat{\alpha}_{j k}=\hat{\beta}_{j k}+\hat{\rho}_{j k}$, como na equação (1.45), onde $(j, k) \in \mathcal{J}_{N}$ está definido em (3.4). Seja $I_{j_{1} k_{1}}^{0}$ o mair intervalo que está incluso em $I_{j+1,2 k}$ (resp $I_{j+1,2 k+1}$ ), com uma decomposição similar à feita em (3.11). Seja $d_{N}$ o número de intervalos de escala grossa na decomposição (3.11) do intervalo $I_{j+1,2 k}$. Suponha que $d_{N}$ é da ordem $\log (N)$. Então:

$$
P\left(\left|\rho_{j k}\right|>t_{j k}\right) \leq O\left(N^{-\lambda}\right)
$$

onde

$$
t_{j k}=\frac{(\log N)^{1 / 2+1 / 2 \theta} \sigma_{\epsilon}}{\sqrt{N}}\left(\sqrt{N 2^{-j_{1}}}+2 N^{\delta^{\prime}+1 / 2}\right) .
$$

O teorema anterior é de grande importância prática. Isso porque se o tradicional limiar universal é usado em dados de séries de tempo, muitas vezes a verdadeira função dos dados é superestimada, devido a existência de coeficientes de perturbação que não foram removidos ao aplicar o limiar.

Como foi dito anteriormente, espera-se que o estimador encontrado esteja o mais próximo possível no sentido do IMSE da projeção $f_{J}(x)$. Para garantir isto é apresentado o teorema a seguir. 
Teorema 3.3.2 Seja $\mathcal{F}=\Lambda^{\beta}(L, B, 0)$, com $1 / 2<\beta<1$. Então para o limiar $t_{j, k}$ dado em (3.21)

$$
\sup _{f \in \mathcal{F}}\left\{E\left\|\hat{f}-f_{J}\right\|_{L_{2}\left(\hat{G}_{N}\right)}^{2}\right\}=o\left(\frac{(\log N)^{1 / 2 \theta+1 / 2}}{N}\right)^{2 \beta /(2 \beta+1)} .
$$

Para a demonstração desse teorema, é útil a decomposição de $\left\|\hat{f}-f_{J}\right\|^{2}$ feita em Delouille (2001), conforme as equações (19) e (20) da página 339. Uma aproximação é:

$$
\begin{aligned}
\left\|\hat{f}-f_{J}\right\|_{L_{2}\left(\hat{G}_{N}\right)}^{2} & \leq 2\left(\sum_{k} E\left[\left(\hat{s}_{j_{0}, k}-\hat{b}_{j_{0}, k}\right)^{2} \phi_{j_{o}, k}(x)\right]+\sum_{j, k} E\left[\left(\eta_{S}\left(\hat{\alpha}_{j, k}, t_{j k}\right)-\alpha_{j, k}\right)^{2} \psi_{j k}(x)\right]\right. \\
& \left.+\sum_{j k} E\left[\left(\alpha_{j, k}-\hat{\beta}_{j k}\right)^{2} \psi_{j k}(x)\right]\right) \\
& =T_{1}+T_{2}+T_{3}
\end{aligned}
$$

Por outro lado, é necessário verificar se o limiar escolhido não é muito grande, se não pode remover o ruído e parte do sinal. Para testar este fato, segundo Delouille (2001), é preciso encontrar um limite superior relacionado com o $l_{2}-$ risco dos coeficientes de detalhes empíricos. Para medir a qualidade do estimador $\alpha^{t}=\eta_{S}\left(\hat{\alpha}_{j, k}, t_{j k}\right)$, pode-se definir um risco ideal segundo Donoho e Johnstone (1994), que é o risco que se obtém de um oráculo para determinar se um coeficiente permanece ou é removido da estimativa.

Considere o modelo (3.1). No domínio das ondaletas, tem-se:

$$
\hat{\alpha}_{j k}=\hat{\beta}_{j k}+\hat{\rho}_{j k}, \quad(j, k) \in \mathcal{J}_{N}
$$

Vamos usar o limiar que depende da escala e localização, $t_{j k}$, encontrado no Teorema 3.3.1, para obter a seguinte desigualdade de risco.

Teorema 3.3.3 No modelo dado em (3.1), considere as suposições 1 a 8. Na decomposição (3.11), suponha que o número $d_{N}$ do intervalo de escala grossa é da ordem $\log (\log (N))$. Considere os coeficientes de detalhe Haar dados em (3.24). Seja $\alpha_{j k}^{t}$ o estimador com limiar suave dos coeficientes, onde $t_{j k}$ é dado no Teorema 3.3.1. Então, um limite superior para o $l_{2}$-risco 
dos coeficientes suavizados é dado por:

$$
\sum_{(j, k) \in \mathcal{J}_{N}} E\left|\alpha_{j, k}^{t}-\alpha_{j, k}\right|^{2} \leq \sum_{(j, k) \in \mathcal{J}_{N}} \frac{c \sigma_{\epsilon}^{2}(\log N)^{1 / \theta+1 / r}}{N^{1-\alpha}}+\min \left(8 \alpha_{j k}^{2}, 4 t_{j k}^{2}\right)
$$

A prova deste teorema segue o Teorema 5.3.6 de Delouille (2002), exceto o Lema 5.6.4. O equivalente para nosso caso é enunciado a seguir:

Lema 3.3.1 Considere o modelo (3.1), as suposições 1-8 do modelo e as suposições do Teorema 3.3.3. Tem-se que:

$$
E\left[\eta_{s}\left(\rho_{j, k}, t_{j, k}\right)^{2}\right]=O\left(\frac{(\log N)^{1 / \theta+1 / r}}{N^{1-\alpha}}\right)
$$

Proposição 3.3.1 Considere o coeficiente de detalhe $\hat{\beta}_{j k}$ como definido em (1.45). Então, uniformemente em $(j, k) \in \mathcal{J}_{N}$, temos

$$
\begin{aligned}
\operatorname{Var}\left(\hat{\beta}_{j k}\right) & \leq \frac{n_{j}}{N}\left|\frac{(\log N)^{1 / 2+1 / 2 \theta} \sigma_{\epsilon}}{\sqrt{N}}\left(\sqrt{N 2^{j_{1}}}+N^{\delta^{\prime}+1 / 2}\right)\right|^{2 \beta} \\
& +2 \frac{n_{j}}{N}\left(8 \alpha^{1 / r}\left|\frac{(\log N)^{1 / 2+1 / 2 \theta} \sigma_{\epsilon}}{\sqrt{N}}\left(\sqrt{N 2^{j_{1}}}+N^{\delta^{\prime}+1 / 2}\right)\right|^{\beta(q+p)} 2^{-j(1+1 / r)}\right)\left(3.2{ }^{\prime}\right.
\end{aligned}
$$

Proposição 3.3.2 Considere os coeficientes $\hat{s}_{j_{0}, k}$ e $\hat{b}_{j_{0}, k}$, definidos em (1.46) e (3.6), respectivamente. Então:

$$
\sum_{k} E\left(\hat{s}_{j_{0}, k}-\hat{b}_{j_{0}, k}\right)^{2} \leq \frac{\sigma_{\epsilon}^{2} n_{j}}{N}\left(1-2^{-j_{0}}\right)+\frac{8 \alpha^{1 / r}}{N} \frac{2^{-j_{o}(1 / p+1 / q-1 / 2)}}{N} .
$$

\subsection{Simulações}

Nesta seção é apresentado um estudo de simulação, feito no software MATLAB com o pacote WAVELAB850. Como realizado no Capítulo 2, os dados consistem de duplas $\left(x_{1}, y_{1}\right),\left(x_{2}, y_{2}\right), \ldots,\left(x_{N}, y_{N}\right)$, que foram geradass de um modelo da forma (3.1). Nele, $X_{t}$ segue um processo $\operatorname{AR}(1)$ da forma dada em (2.24), onde $u_{t}$ e $\epsilon_{t}$ têm distribuição Normal padrão e são independentes para todo $t \in \mathcal{Z}$.

A amostra da variável $X_{t}$ foi obtida da seguinte forma. Primeiro é tomado $x_{0}=250 \mathrm{e}$ $x_{0}=1025$, para $N=512$ e $N=1024$, respectivamente. A seguir é gerado um valor $u_{1}$ de uma 
normal padrão e é obtido o valor

$$
x_{1}=0,2 x_{0}+u_{1}
$$

Na sequência, é gerado um novo valor $u_{2}$ de uma normal padrão e é calculado o valor $x_{2}=$ $0,2 x_{1}+u_{2}$, assim sucessivamente até gerar 5000 valores de $x_{t}$. Depois são escolhidos os últimos n valores, para $N=512$ e $N=1024$, e os outros valores são descartados. A seguir, os valores de $Y_{t}$ foram calculados sem ruído, ou seja $y_{t}=f\left(x_{t}\right)$, para $t=1,2, \ldots, N$. Estes pontos são gerados uma única vez, tornando-os fixos nas simulações. Depois foram gerados os ruídos $\epsilon_{t}$ com a distribuição $\mathcal{N}(0,1)$ e adicionados aos valores de $y_{t}$ (esses valores $\epsilon_{t}$ foram gerados para cada replicação).

Neste estudo de simulações são comparados os estimadores obtidos ao aplicar ondaletas adaptativas de Haar com o limiar comum $t_{j k}=\sigma_{j k} \sqrt{\log N}$ e o limiar obtido para dados $\alpha$-mixing, proposto neste trabalho, dado por $t_{j k}=\sigma_{j k}(\log N)^{1 / 2+1 / 2 \theta}$, onde o valor de $\theta$ considerdo é $\theta=9 / 8$. Estes estimadores também são comparados com dois níveis de ruído $s n r=1$ (ruído maior) e $s n r=7$ (ruído menor), onde $s n r$ é dado pela expressão (2.26).

Um dos assuntos de interesse prático é como estimar $\sigma_{j k}=\operatorname{var}\left(\beta_{j k}\right)$, a partir dos dados. Espera-se que o algoritmo de três passos dado por Delouille (2001) forneça um estimador razoável. Os passos deste algoritmo são:

1 Fazer uma regressão robusta piloto usada para obter os resíduos;

2 Estimar a variância local por outra regressão robusta, usando os resíduos quadráticos;

3 Usar as estimativas obtidas no passo anterior na aplicação dos limiares.

Na Tabela 3.1 são apresentados os valores do RMSE para as simulações feitas para as ondaletas adaptativas de Haar. Os coeficientes foram limiarizados com o limiar suave a partir do nível $j_{0}=2$. Observa-se que, para o nível de ruído $s n r=1$, os valores do RMSE são maiores que para o nível de snr=7. Além disso, observa-se que o procedimento tem um RMSE menor para as estimativas obtidas com o limiar para dados $\alpha$-mixing. O procedimento apresenta um desempenho mais baixo para a função de prova $f(x)=$ Doppler .

Nas figuras A.7 e A.8 no Apêndice A, são apresentados os gráficos de boxplot para os valores 

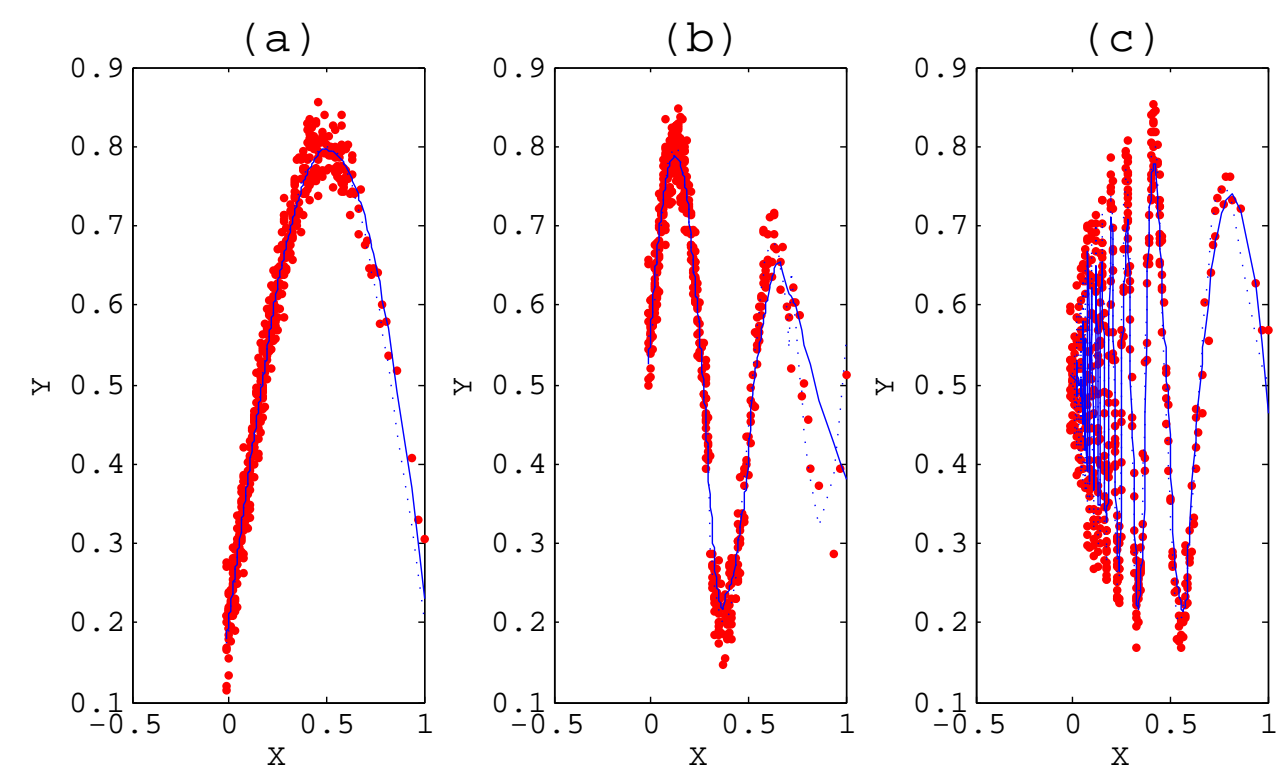

Figura 3.1: Os pontos representam o planejamento aleatório do modelo 3.1. A linha tracejada representa a equação $Y_{t}=f\left(X_{t}\right)$, a verdadeira função. A linha contínua representa a função estimada com ondaletas adaptativas. As funções são: (a) função Seno com $r s n r=7$, (b) função $\mathrm{f}(\mathrm{x})$ Heavisine com $r s n r=7$, (c) Função Doppler com rsnr=7

\begin{tabular}{cccccc}
\hline & \multicolumn{2}{c}{ Haar-mixing } & & \multicolumn{2}{c}{ Haar-independente } \\
\cline { 2 - 3 } \cline { 5 - 6 } & $\mathrm{SNR}=1$ & $\mathrm{SNR}=7$ & & $\mathrm{SNR}=1$ & $\mathrm{SNR}=7$ \\
\hline Seno & & & & & \\
$\mathrm{N}=512$ & $3,35 \times 10^{-4}$ & $9,62 \times 10^{-5}$ & & $3,27 \times 10^{-2}$ & $7,94 \times 10^{-4}$ \\
$\mathrm{~N}=1024$ & $1,94 \times 10^{-5}$ & $4,27 \times 10^{-5}$ & & $3,14 \times 10^{-2}$ & $6,44 \times 10^{-4}$ \\
HeaviSine & & & & \\
$\mathrm{N}=512$ & $7,35 \times 10^{-3}$ & $8,43 \times 10^{-4}$ & & $3,63 \times 10^{-2}$ & $6,16 \times 10^{-4}$ \\
$\mathrm{~N}=1024$ & $3,65 \times 10^{-3}$ & $2,57 \times 10^{-4}$ & & $2,95 \times 10^{-2}$ & $6,36 \times 10^{-4}$ \\
Doppler & & & & & \\
$\mathrm{N}=512$ & $1,71 \times 10^{-2}$ & $2,10 \times 10^{-3}$ & & $3,00 \times 10^{-3}$ & $1,62 \times 10^{-3}$ \\
$\mathrm{~N}=1024$ & $1,71 \times 10^{-2}$ & $3,57 \times 10^{-4}$ & & $3,98 \times 10^{-2}$ & $8,39 \times 10^{-4}$ \\
\hline
\end{tabular}

Tabela 3.1: RMSE do estudo de simulação.

de MXDV obtidos com as simulações feitas para ondaletas adaptativas. Para $s n r=1$ e para $s n r=7$, os valores de MXDV são maiores nas estimativas obtidas com o limiar usual. Por outro lado, tem-se que a função Doppler foi a que apresentou valores de MXDV maiores.

Na Figura 3.1 são apresentados os gráficos das funções usadas para testar o desempenho do procedimento de ondaletas adaptativas de Haar. Observa-se que o procedimento consegue capturar a tendência não linear dos dados; nas regiões onde se tem menos pontos amostrados, 
o estimador $\hat{f}$ fica um pouco mais afastado da $f$ verdadeira.

\subsection{Provas}

Para demonstrar os teoremas, necessitamos dos seguintes lemas:

Lema 3.5.1 Seja $\gamma>0, c>0$ e $\left(Z_{i}\right)_{i \in \mathbb{Z}}$ um processo estacionário mixing com coeficiente mixing satisfazendo $\alpha_{m} \leq \gamma e^{-c|m|^{\theta}}$. Seja $N \in \mathbb{N}^{*}, h: \mathbb{R} \rightarrow \mathbb{R}$ uma função mensurável, tal que, para todo $i \in \mathbb{Z}, U_{i}=h\left(Z_{i}\right)$. Suponha que $E\left[U_{i}\right]=0$ e que existe uma constante $M>0$ satisfazendo $\left|U_{i}\right| \leq M<\infty$. Então, para $m \in\{1,2, \ldots, N\}$ e para $c_{\lambda}>4 M m / N$ tem-se

$$
P\left(\left|\frac{1}{N} \sum_{t=1}^{N} U_{i}\right|>c_{\lambda}\right) \leq 4 \exp \left\{\frac{-N c_{\lambda^{2}}}{m\left(64 E\left[U_{i}^{2}\right]+8 M c_{\lambda} / 3\right)}\right\}+4 \gamma \frac{N}{m} \exp \left\{-c|m|^{\theta}\right\}
$$

\section{Prova:}

Para qualquer inteiro $i$, seja $U_{i}=\sum_{i m-m<l<i m} Z_{l}, Z_{l}=0$ se $l>N, U_{2 i}=U_{2 i-1}=0$ q.c se $i>\left\lceil\frac{N}{2 m}\right\rceil+1$. Então,

$$
\sup _{j \leq N}\left|\sum_{i=1}^{j} Z_{i}\right| \leq M m+\sup _{j>0}\left|\sum_{i=1}^{j} U_{i}\right|
$$

mas como as variáveis $U_{i}$ são mixing, vamos usar a técnica de acoplamento para variáveis $\alpha$ mixing para conseguir sequências de v.a independentes, no entanto com a mesma distribuição de $U_{i}$. Seja $\vartheta_{i}$ uma sequência de variáveis aleatórias independentes com distribuição uniforme sobre $[0,1]$, independente de $U_{i}$. Logo, pelo Teorema 4 (Rio, 1995), para todo $i$ existe uma função mensurável $F_{i}$ tal que $U_{i}^{*}=F_{i}\left(U_{1}, \ldots, U_{i-1}, U_{i}, \vartheta_{i}\right)$ satisfaz as condições do Teorema 4 (Rio, 1995) $\operatorname{com} \mathcal{A}=\sigma\left(U_{l}: l<i-1\right)$. A sequência $\left\{U_{i}^{*}\right\}_{i>0}$ assim definida tem as seguintes propriedades:

1. Para qualquer inteiro positivo $i$, a variável aleatória $U_{i}^{*}$ tem a mesma distribuição que $U_{i}$;

2. A sequência de variáveis aleatórias $\left\{U_{2 i}^{*}\right\}_{i>0}$ é independente e a sequência $\left\{U_{2 i-1}^{*}\right\}_{i>0}$ também é independente;

3. Além disso,

$$
\sum_{i>0} E\left[\left|U_{i}-U_{i}^{*}\right|\right] \leq 4 n M \alpha_{m}
$$


Logo,

$$
\sup _{j>0}\left|\sum_{i=1}^{j} U_{i}\right| \leq \sup _{j>0}\left|\sum_{i=1}^{j} U_{2 i}^{*}\right|+\sup _{j>0}\left|\sum_{i=1}^{j} U_{2 i-1}^{*}\right|+\sum_{i>0}\left|U_{i}-U_{i}^{*}\right| .
$$

Considerando a propriedade 3 e a desigualdade de Markov, tem-se que:

$$
P\left(\sum\left|U_{i}-U_{i}^{*}\right|>M m\right) \leq 4 N \frac{\alpha_{m}}{m}
$$

Por outro lado, como a sequência $U_{2 i}^{*}$ é independente, pode-se aplicar a desigualdade de Berstein para v.a independentes, obtendo-se

$$
P\left(\sup _{j>0}\left|\sum_{i=1}^{j} U_{2 i}^{*}\right|>\frac{N c_{\lambda}}{4}\right) \leq 2 \exp \left\{\frac{-N^{2} c_{\lambda}^{2}}{16\left(2 \sum E\left[U_{2 i}^{* 2}\right]+2 M N c_{\lambda} / 12\right)}\right\}
$$

Pela propriedade 1, tem-se que $\operatorname{Var}\left(U_{2 i}^{*}\right) \leq \operatorname{Var}\left(U_{i}\right)=2 m E\left[U_{i}^{2}\right]$ e $\left\|U_{2 i}^{*}\right\|_{\infty} \leq M m$, assim $\sum_{t=1}^{N} \operatorname{Var}\left(U_{i}\right)=\sum_{t=1}^{N} E\left[U_{2 i}^{* 2}\right]=2 N m E\left[U_{i}^{2}\right]$. Logo, substituindo em (3.33), obtém-se:

$$
\begin{aligned}
& P\left(\left|\sum U_{2 i}^{*}\right|>\frac{N c_{\lambda}}{4}\right) \leq 2 \exp \left\{\frac{-N^{2} c_{\lambda}^{2}}{16\left(4 N m E\left[U_{i}^{2}\right]+2 M m N c_{\lambda} / 12\right)}\right\}, \\
& =2 \exp \left\{\frac{-N^{2} c_{\lambda}^{2}}{N m\left(64 E\left[U_{i}^{2}\right]+8 M c_{\lambda} / 3\right)}\right\}, \\
& =2 \exp \left\{\frac{-N c_{\lambda}^{2}}{m\left(64 E\left[U_{i}^{2}\right]+8 M c_{\lambda} / 3\right)}\right\} \text {. }
\end{aligned}
$$

Aplicando o mesmo tratamento para a sequência de variáveis $U_{2 i-1}^{*}$, obtemos o mesmo resultado. Desta forma, temos de (3.32) e (3.34) o resultado esperado.

O lema anterior confere uma desigualdade do tipo Bernstein para variáveis $\alpha$-mixing. E os dois lemas seguintes permitem mostrar como é usada a técnica de acoplamento para encontrar um limiar adequado para regressão com dados dependentes.

Lema 3.5.2 Seja $\mathcal{I}$ um intervalo aleatório tal que

$$
I_{j_{1}, k_{1}}^{0} \cup I_{j_{2}, k_{2}}^{0} \cup, \ldots \cup I_{j_{d}, k_{d}}^{0} \subseteq \mathcal{I}
$$


em que o intervalo $I_{j, k}^{0}$ é dado em (3.9). Sob as suposições do modelo, tem-se

$$
P\left(\sum_{i=1}^{d(\mathcal{I})}\left|\sum_{t} \epsilon_{t} 1_{j_{i}, k_{i}}^{0}\left(X_{t}\right)\right|>c_{\lambda} N^{1 / 2}(\log (N))^{1 / 2+1 / 2 \theta} \sigma_{\epsilon} 2^{j_{i} / 2}\right) \leq O\left(N^{-\lambda}\right),
$$

onde $1_{j_{i}, k_{i}}^{0}$ é a função indicadora do intervalo $I_{j_{i}, k_{i}}^{0}$, definido em (3.10), e $c_{\lambda}$ é uma constante que depende de $\lambda$.

\section{Prova:}

Pelas suposições 5 e 8 do modelo, sabe-se que $\left\{X_{t}\right\}_{t=1}^{N}$ é uma sequência de v.a estacionárias, com coeficiente mixing associado dado por $\alpha_{m} \leq \gamma e^{-c|m|^{\theta}}$. Seja $U_{t}=h\left(X_{t}\right)=\epsilon_{t} 1_{j_{i}, k_{i}}^{0}\left(X_{t}\right)$, $i=1,2, \ldots, d(\mathcal{I})$. Com $E\left[U_{t}\right]=0$ e $\operatorname{Var}\left(U_{t}\right)=\sigma_{\epsilon}^{2} 2^{-j_{i}}$. Além disso $\left|X_{t}\right| \leq K$, pela suposição 4 do modelo, de onde $\left|U_{t}\right| \leq K N^{\delta^{\prime}}=M^{*}$.

Agora, estão satisfeitas todas as condições para aplicar o Lema 3.5.1.

$$
P\left(\left|\sum_{t} U_{t}\right|>N c_{\lambda}\right) \leq 4 \exp \left\{\frac{-16 N c_{\lambda}^{2}}{m\left(64 N E\left[U_{t}^{2}\right]+8 c_{\lambda} M^{*} / 3\right)}\right\}+4 \gamma \frac{N}{m} e^{-c m^{\theta}}
$$

e fazendo $m=s(\log (N))^{1 / \theta} \operatorname{com} s>0$ e $c_{\lambda}^{2}=4 \lambda N^{-1} \sigma_{\epsilon}^{2} 2^{-j_{i}}\left(\log (N)^{1+1 / \theta}\right)$, ao substituir em (3.37) temos:

$$
\begin{aligned}
P\left(\left|\sum_{t} U_{t}\right|>N c_{\lambda}\right) & \leq 4 \exp \left\{\frac{-16 N\left(4 \lambda N^{-1} \sigma_{\epsilon}^{2} 2^{-j_{i}}\right)(\log (N))^{1+1 / \theta}}{s(\log (N))^{1 / \theta}\left(64 \sigma_{\epsilon}^{2} 2^{-j_{i}}+8\left(4 \lambda N^{-1} \sigma_{\epsilon}^{2} 2^{-j_{i}}\left(\log (N)^{1+1 / \theta}\right)\right)^{1 / 2} K N^{\delta^{\prime}} / 3\right)}\right\} \\
& +4 \gamma \frac{N}{s(\log (N))^{1 / \theta}} e^{-c\left(s \log (N)^{1 / \theta}\right)^{\theta}} \\
& \leq 4 \exp \left\{\frac{-16 N\left(4 \lambda N^{-1} \sigma_{\epsilon}^{2} 2^{-j_{i}}\right)(\log (N))^{1+1 / \theta}}{64 s(\log (N))^{1 / \theta} \sigma_{\epsilon}^{2} 2^{-j_{i}}}\right\} \\
& +4 \exp \left\{\frac{-16 N\left(4 \lambda N^{-1} \sigma_{\epsilon}^{2} 2^{-j_{i}}\right)(\log (N))^{1+1 / \theta}}{8 s(\log (N))^{1 / \theta}\left(4 \lambda N^{-1} \sigma_{\epsilon}^{2} 2^{-j_{i}}\right)^{1 / 2} K N^{\delta^{\prime}} / 3}\right\}
\end{aligned}
$$




$$
\begin{aligned}
& +4 \gamma \frac{N}{s(\log (N))^{1 / \theta}} e^{-c s^{\theta}(\log (N))} \\
& \leq 4 \exp \{-\lambda(\log (N))\}+4 \exp \left\{\frac{-12 N^{1 / 2-\delta^{\prime}} \lambda^{1 / 2} s \sigma_{\epsilon} 2^{-j_{i} / 2}(\log (N))^{1-1 / \theta}}{s K N^{\delta^{\prime}}}\right\} \\
& +4 \gamma \frac{N^{1-c}}{s(\log (N))^{1 / \theta}} \\
& \leq N^{-\lambda}+4 \exp \left\{-12 \sigma_{\epsilon} \lambda^{1 / 2} s(\log (N))^{1 / 2-1 / 2 \theta} N 2^{j_{i} / 2}\right\}+4 \gamma \frac{N^{1-c}}{s(\log (N))^{1 / \theta}} \\
& \leq N^{-\lambda}+4 \exp \left\{-12 \sigma_{\epsilon} \lambda^{1 / 2} s(\log (N))^{1 / 2-1 / 2 \theta}\right\}+4 \gamma \frac{N^{1-c}}{s(\log (N))^{1 / \theta}} \\
& \leq C_{1} N^{-\lambda}+c_{2} N^{-\lambda}+C_{3} N^{1-c} \leq C N^{-\lambda} .
\end{aligned}
$$

Logo, somando sob $i=1, \ldots, N$ tem-se que $\sum_{i=1}^{d} 2^{-j_{i}}=O\left(2^{j_{1}}\right) \mathrm{e}$

$$
c_{\lambda}=\left(4 \lambda N^{-1} \sigma_{\epsilon}^{2} 2^{-j_{1}} s\left(\log (N)^{1+1 / \theta}\right)\right)^{1 / 2}
$$

Lema 3.5.3 Seja $\mathcal{I}$ um intervalo aleatório tal que

$$
\mathcal{I} \subseteq I_{j_{1}, k_{1}}^{0} \cup \ldots \cup I_{j_{d}, k_{d}}^{0} \cup I_{j^{*}, l_{1}}^{0} \cup I_{j^{*}, l_{2}}^{0}
$$

em que os $I_{j_{i}, k_{i}}^{0}$ são definidos em (1.32) e $2^{j^{*}} \leq N \leq 2^{j^{*}+1}$. Sob as suposições do modelo temos

$$
P\left(\sum\left|\epsilon_{t}\right| 1_{I_{j^{*}, l_{1}}^{0} \cup I_{j^{*}, l_{2}}^{0}}\left(X_{t}\right)>c\left(\lambda^{1 / 2}(\log (N))^{1 / 2+1 / 2 \theta} \sigma_{\epsilon} 2^{j^{*} / 2}+N^{\delta^{\prime}}+N 2^{-j^{*}}\right)\right) \leq c_{\lambda} N^{-\lambda} .
$$

\section{Prova:}

A prova segue o esquema de Delouille (2001) para mostrar o seu lema 5.6.2. O objetivo é avaliar a probabilidade $P\left(\left|\epsilon_{t}\right| 1_{I_{j^{*}, l_{i}}^{0}}\left(X_{t}\right)>c\right)$ para $i=1,2$, mas não é conhecido com precisão o intervalo $I_{j^{*}, l_{i}}^{0}$. Como é preciso garantir o recobrimento do intervalo $\mathcal{I}$, é tomado o máximo sobre todas as localizações de $l$. Embora dado um intervalo $I_{j^{*} l_{i}}^{0}$, este não está necessariamente 
incluído em $\mathcal{I}$. Portanto, é tomado o limite superior em $\left|\epsilon_{t}\right|$,

$$
\begin{aligned}
P\left(\sum_{t}\left|\epsilon_{t}\right| 1_{I_{j^{*}, l_{i}}^{0}}\left(X_{t}\right)>c\right) & \leq P\left(\sum_{t} N^{\delta^{\prime}} 1_{I_{j^{*}, l_{i}}^{0}}\left(X_{t}\right)>c\right) \\
& \leq P\left(\max _{l=1,2, \ldots, 2^{j^{*}}} \sum_{t} N^{\delta^{\prime}} 1_{I_{j^{*} l}^{0}}\left(X_{t}\right)>c\right) \\
& \leq \sum_{t=1}^{2^{j^{*}}} P\left(N^{\delta^{\prime}} \sum_{t} 1_{I_{j^{*}, l}^{0}}\left(X_{t}\right)>c\right) \\
& \leq N P\left(N^{\delta^{\prime}} \sum_{t} 1_{I_{j^{*}, l}^{0}}\left(X_{t}\right)>c\right) .
\end{aligned}
$$

É preciso encontrar um $a_{\lambda}$ tal que

$$
P\left(\sum_{t} 1_{I_{j^{*}, l}^{0}}\left(X_{t}\right)>a_{\lambda}\right) \leq c N^{-\lambda}
$$

o que significa que $c_{\lambda}=N^{\delta^{\prime}} a_{\lambda}$. Seja $W_{t}=1_{I_{j^{*} l_{1}}^{0}}$, uma variável aleatória com média diferente de zero, portanto não pode ser aplicado o Lema 3.5.3. Mas a variável $W_{t}^{*}=1_{I_{j^{*} l_{1}}^{0}}-2^{-j^{*}}$ tem média zero, e com isso, o Lema pode ser aplicado:

$$
\begin{aligned}
& P\left(\sum_{t} W_{t}^{*}>N b_{\lambda}\right) \\
\leq & 4 \exp \left\{\frac{-16 N^{2} b_{\lambda}^{2}}{m\left(64 N E\left[W_{t}^{* 2}\right]+8 b_{\lambda} M / 3\right)}\right\}+4 \gamma \frac{N}{m} \exp \left\{-c m^{\theta}\right\} \\
\leq & 4 \exp \left\{\frac{-16 N b_{\lambda}^{2}}{64 m E\left[W_{t}^{*}\right]}\right\}+4 \exp \left\{\frac{-16 N b_{\lambda}^{2}}{8 m b_{\lambda} M / 3}\right\}+4 \gamma \frac{N}{m} \exp \left\{-c m^{\theta}\right\} .
\end{aligned}
$$

Fazendo $m=s(\log (N))^{1 / \theta}, M=K$ e $b_{\lambda}^{2}=4 N^{-1} \lambda s(\log (N))^{1+1 / \theta} \sigma_{W^{*}}^{2}$, o resultado é

$$
\begin{aligned}
& P\left(\sum_{t} W_{t}^{*}>N b_{\lambda}\right) \\
& \leq 4 N^{-\lambda}+4 \exp \left\{\frac{-16 N\left(4 N^{-1} s(\log (N))^{1+1 / \theta} \sigma_{W^{*}}^{2}\right)^{1 / 2}}{8 s(\log (N))^{1 / \theta} K / 3}\right\}+4 \gamma \frac{N^{1-c}}{s(\log (N))^{1 / \theta}} \\
& \leq N^{-\lambda}+4 \exp \left\{\frac{-24 \sigma_{W}^{2} \lambda(\log (N))}{K}\right\}+4 \gamma \frac{N^{1-c}}{s(\log (N))^{1 / \theta}} \\
& \leq C N^{-\lambda} .
\end{aligned}
$$


Por outro lado, temos:

$$
\begin{aligned}
P\left(\sum_{t} W_{t}^{*}>N b_{\lambda}\right) & =P\left(\sum_{t} W_{t}-N 2^{-j^{*}}>N b_{\lambda}\right) \\
& =P\left(\sum_{t} 1_{I_{j^{*}, l}^{0}}\left(X_{t}\right)>N b_{\lambda}+N 2^{-j^{*}}\right)
\end{aligned}
$$

$\log 0 a_{\lambda}=N b_{\lambda}+N 2^{j^{*}}=N\left(4 N^{-1} \lambda s(\log (N))^{1+1 / \theta} \sigma_{W^{*}}^{2}\right)+N 2^{-j^{*}} \mathrm{e}$

$c_{\lambda}=N^{\delta^{\prime}+1}\left(\left(4 N^{-1} \lambda s(\log (N))^{1+1 / \theta} \sigma_{W^{*}}^{2}\right)^{1 / 2}+2^{-j^{*}}\right)$.

\section{Teorema 3.3.1:}

Seja $\mathcal{I}=I_{j k}$ um dos intervalos aleatórios tal que $(j, k) \in \mathcal{J}_{N}$, com $\mathcal{J}_{N}$ definido tal qual em (3.4). Temos a aproximação $\mathcal{I} \subseteq I_{j_{1} k_{1}}^{0} \cup \ldots \cup I_{j_{d}, k_{d}}^{0} \cup I_{j^{*} l_{1}}^{0} \cup I_{j^{*} l_{2}}^{0}$, com $I_{j_{1} k_{1}}^{0}$ o maior intervalo usado para recobrir $\mathcal{I}$. Agora vamos provar que

$$
P\left(\sum_{t} \epsilon_{t} 1_{\mathcal{I}}\left(X_{t}\right)>c_{\lambda}\left(\frac{(\log N)^{1 / 2+1 / 2 \theta} \sigma_{\epsilon}}{\sqrt{N}}\left(N^{1 / 2} 2^{j_{1} / 2}+2 N^{\delta^{\prime}}\right)\right)\right) \leq O\left(N^{-\lambda}\right) .
$$

Usando a decomposição (3.16) com uma constante genérica $c$, tem-se:

$$
P\left(\left|\sum_{t} \epsilon_{t} 1_{\mathcal{I}}\left(X_{t}\right)\right|>c\right) \leq P\left(\sum_{i=1}^{d(\mathcal{I})}\left|\sum_{t} \epsilon_{t} 1_{j_{i} k_{i}}\left(X_{t}\right)\right|+\sum_{t}\left|\epsilon_{t} 1_{I_{j^{*} l_{1}}^{0} \cup I_{j^{*} l_{2}}^{0}}\left(X_{t}\right)\right|>c\right)
$$

Pelo Lema 3.5.2 conclui-se:

$$
P\left(\sum_{i=1}^{d(\mathcal{I})}\left|\sum_{t} \epsilon_{t} 1_{j_{i} k_{i}}\left(X_{t}\right)\right|>c_{\lambda}\left(N^{1 / 2} \sigma_{\epsilon} 2^{j_{1} / 2}(\log N)^{1 / 2+1 / 2 \theta}\right)\right) \leq O\left(N^{-\lambda}\right)
$$

e pelo Lema 3.5.3 temos que:

$$
P\left(\sum_{t}\left|\epsilon_{t}\right| 1_{I_{j^{*} l_{1}}^{0} \cup I_{j^{*} l_{2}}^{0}}\left(X_{t}\right)>c_{\lambda} N^{\delta^{\prime}+1}\left(N^{-1 / 2}(\log N)^{1 / 2+1 / 2 \theta} \sigma_{w^{*}}+2^{-j^{*}}\right)\right) \leq O\left(N^{-\lambda}\right)(3.5
$$

onde $\sigma_{w^{*}}=\left(2^{j^{*} / 2}\left(1-2^{j^{*}}\right)^{1 / 2}\right)$. Desta forma:

$$
\begin{aligned}
& t_{j k}=c_{\lambda}\left(N^{1 / 2} \sigma_{\epsilon} 2^{j_{1} / 2}(\log N)^{1 / 2+1 / 2 \theta}\right) \\
& +\quad c_{\lambda} N^{\delta^{\prime}+1}\left(N^{-1 / 2}(\log N)^{1 / 2+1 / 2 \theta} \sigma_{w^{*}}+2^{-j^{*}}\right) \leq O\left(N^{-\lambda}\right),
\end{aligned}
$$


como foi descrito anteriormente $2^{j^{*}}<N<2^{j^{*}+1}$, portanto $\left(1-2^{-j^{*}}\right)=o(1)$ e $N 2^{-j^{*}}=o(1)$, assim

$$
t_{j k}=\frac{(\log N)^{1 / 2+1 / 2 \theta} \sigma_{\epsilon}}{\sqrt{N}}\left(\sqrt{N 2^{-j_{1}}}+2 N^{\delta^{\prime}+1 / 2}\right) .
$$

\section{Proposição 3.3.1:}

Pela definição de $\hat{\alpha}_{j k}$ em (1.45), tem-se

$$
\hat{\alpha}_{j k}=\hat{\beta}_{j k}+\hat{\rho}_{j k}
$$

Estamos interessados em $\hat{\beta}_{j k}$; logo o coeficiente pode-se decompor da seguinte forma:

$$
\begin{aligned}
\hat{\beta}_{j k} & =\frac{1}{N} \sum_{t=1}^{N} f\left(X_{t}\right)\left(1_{j+1,2 k}\left(X_{t}\right)-1_{j+1,2 k+1}\left(X_{t}\right)\right) \\
& =D_{1}-D_{2}
\end{aligned}
$$

$\log 0$

$$
\operatorname{Var}\left(\hat{\beta}_{j k}\right) \leq 2\left(\operatorname{Var}\left(D_{1}\right)+\operatorname{Var}\left(D_{2}\right)\right)
$$

e

$$
\operatorname{Var}\left(D_{1}\right) \leq \frac{1}{N n_{j}} \operatorname{Var}\left(\sum_{t=1}^{N} f\left(X_{t}\right) 1_{j+1,2 k}\left(X_{t}\right)\right)
$$

Para facilidade na notação são trocados os subíndices $(j+1,2 k)$ por $(j, k)$, logo

$$
\begin{aligned}
\operatorname{Var}\left(\sum_{t=1}^{N} f\left(X_{t}\right) 1_{j+1,2 k}\left(X_{t}\right)\right) & =\sum_{t=1}^{N} \operatorname{Var}\left(f\left(X_{t}\right) 1_{j, k}\left(X_{t}\right)\right) \\
& +2 \sum_{t} \sum_{s} \operatorname{Cov}\left(f\left(X_{t}\right) 1_{j, k}\left(X_{t}\right) ; f\left(X_{s}\right) 1_{j, k}\left(X_{s}\right)\right) .
\end{aligned}
$$

Para $(k-1) n_{j}+1 \leq t \leq k n_{j}$,

$$
\sum_{t=1}^{N} \operatorname{Var}\left(f\left(X_{t}\right) 1_{j, k}\left(X_{t}\right)\right)=\sum_{t=1}^{N} \operatorname{Var}\left(f\left(X_{t}\right)\right),
$$


como definido em Delouille (2001), sejam $U_{t}=G_{x}\left(X_{t}\right), \mathrm{t}=1,2, \ldots, \mathrm{N}$, e $h(u)=f\left(G_{x}^{-1}(u)\right)$. Para $X_{t} \in I_{j, k}$, tem-se

$$
\operatorname{Var}\left(f\left(X_{t}\right)\right) \leq \operatorname{Var}\left(h\left(U_{(t)}\right)\right) \leq E\left[\left(h\left(U_{(t)}\right)-h(t / N)\right)^{2}\right]
$$

e pelos Lema 3 de Delouille (2002) obtemos

$$
E\left[\left(h\left(U_{(t)}\right)-h(t / N)\right)^{2}\right] \leq \tilde{H}^{2} E\left[\left(U_{t}-t / N\right)^{2 \beta}\right]
$$

Deste modo, pelo Lema 3.3 (Wang et al., 2011)

$$
P\left(\left|U_{(t)}-\frac{t}{N}\right| \leq \epsilon_{N}\right)=1
$$

para $N$ suficientemente grande e, no nosso caso, para $\epsilon_{n}=\frac{(\log N)^{1 / 2+1 / 2 \theta} \sigma_{\epsilon}}{\sqrt{N}}\left(\sqrt{N 2^{j_{1}}}+N^{\delta^{\prime}+1 / 2}\right)$, obtemos:

$$
\tilde{H}^{2} E\left[\left(U_{t}-t / N\right)^{2 \beta}\right] \leq \tilde{H}^{2}\left|\frac{(\log N)^{1 / 2+1 / 2 \theta} \sigma_{\epsilon}}{\sqrt{N}}\left(\sqrt{N 2^{j_{1}}}+N^{\delta^{\prime}+1 / 2}\right)\right|^{2 \beta}
$$

Por outro lado, como $f\left(X_{t}\right) 1_{j, k}\left(X_{t}\right)$ é uma variável $\alpha$-mixing, se tem que

$$
\begin{aligned}
\operatorname{Cov}\left(f\left(X_{t}\right) 1_{j, k}\left(X_{t}\right) ; f\left(X_{s}\right) 1_{j, k}\left(X_{s}\right)\right) \leq & 8 \alpha^{1 / r}\left(E\left[\left|f\left(X_{t}\right) 1_{j, k}\left(X_{t}\right)\right|^{p}\right]\right)^{1 / p} \\
& \left(E\left[\left|f\left(X_{s}\right) 1_{j, k}\left(X_{s}\right)\right|^{q}\right]\right)^{1 / q}
\end{aligned}
$$

e como as duas esperanças que envolvem a expressão (3.68) podem ser tratadas de forma análoga, só é calculada com detalhe $E\left[\left|f\left(X_{t}\right) 1_{j, k}\left(X_{t}\right)\right|^{p}\right]^{1 / p}$. Primeiro vamos limitar a esperança:

$$
\left(E\left[\left|f\left(X_{t}\right) 1_{j, k}\left(X_{t}\right)\right|^{p}\right]\right) \leq E\left[\left|f\left(X_{t}\right)^{p} 1_{j, k}\left(X_{t}\right)^{p}\right|\right]
$$

Agora, chamemos $X^{*}=\left|f\left(X_{t}\right)\right|^{p}<\infty$ e $Y^{*}=\left|1_{j k}\left(X_{t}\right)\right|^{p}<\infty$, logo pode-se aplicar de novo a desigualdade de Hölder, assim que

$$
E\left[\left|X^{*} Y^{*}\right|\right] \leq E^{1 / p}\left|X^{*}\right|^{p} E^{1 / q^{*}}\left|Y^{*}\right|^{q^{*}},
$$


onde $1 / q^{*}=(1 / q+1 / r)$. A seguir, vamos calcular cada uma das esperanças envolvidas na equação anterior.

$$
E\left|X^{*}\right|^{p}=E\left|f\left(X_{t}\right)\right|^{p^{2}}
$$

como feito em Delouille (2001), toma-se $\tau=\left(k-\frac{1}{2}\right) n_{j}$, tal que $q_{\tau}$ é o ponto médio do intervalo $I_{j k}, \operatorname{logo}$

$$
E\left|f\left(X_{t}\right)\right|^{p^{2}} \leq\left(f(\tau / N)^{p^{2}}+E\left[f\left(X_{t}\right)-f(\tau / N)\right]^{p^{2}}\right)
$$

como definido anteriormente

$$
\left(E\left[f\left(X_{t}\right)-f(\tau / N)\right]\right)^{p^{2}}=\left(E\left[h\left(U_{(t)}\right)-h(\tau / N)\right]\right)^{p^{2}},
$$

assim, pelo Lema 3 de Delouille (2002) obtém-se:

$$
\left(\left[h\left(U_{(t)}\right)-h(\tau / N)\right]\right)^{p^{2}} \leq \tilde{H}^{p^{2}}\left|U_{(t)}-\tau / N\right|^{p^{2} \beta}
$$

e para todo

$$
\begin{aligned}
U_{(t)}, \tau / N \in[ & \frac{(k-1) n_{j}-1}{N}-\frac{(\log N)^{1 / 2+1 / 2 \theta} \sigma_{\epsilon}}{\sqrt{N}}\left(\sqrt{N 2^{j_{1}}}+N^{\delta^{\prime}+1 / 2}\right) \\
& \left.; \frac{(k) n_{j}+1}{N}+\frac{(\log N)^{1 / 2+1 / 2 \theta} \sigma_{\epsilon}}{\sqrt{N}}\left(\sqrt{N 2^{j_{1}}}+N^{\delta^{\prime}+1 / 2}\right)\right],
\end{aligned}
$$

para N suficientemente grande. Por outro lado tem-se que

$$
\begin{gathered}
E\left[\mid Y^{*} q^{q^{*}}\right]^{1 / q^{*}}=E\left[\left|1_{j k}\left(X_{t}\right)\right|^{p q^{*}}\right]^{1 / q^{*}}=2^{-j / q^{*}} \\
E\left[\left|f\left(X_{s}\right) 1_{j, k}\left(X_{s}\right)\right|^{p}\right] \leq \tilde{H}^{p^{2}}\left|U_{(t)}-\tau / N\right|^{\beta p^{2}} 2^{-j / q^{*}} \\
\leq \tilde{H}^{p^{2}}\left(\frac{(\log N)^{1 / 2+1 / 2 \theta} \sigma_{\epsilon}}{\sqrt{N}}\left(\sqrt{N 2^{j_{1}}}+N^{\delta^{\prime}+1 / 2}\right)\right)^{\beta p^{2}} 2^{-j / q^{*}}
\end{gathered}
$$


De forma análoga, pode-se mostrar que

$$
E\left[\left|f\left(X_{s}\right) 1_{j, k}\left(X_{s}\right)\right|^{q}\right] \leq \tilde{H}^{q^{2}}\left(\frac{(\log N)^{1 / 2+1 / 2 \theta} \sigma_{\epsilon}}{\sqrt{N}}\left(\sqrt{N 2^{j_{1}}}+N^{\delta^{\prime}+1 / 2}\right)\right)^{\beta q^{2}} 2^{-j / p^{*}}
$$

onde $1 / p^{*}=(1 / q+1 / r)$. Assim conclui-se que:

$$
\begin{aligned}
& \operatorname{Cov}\left(f\left(X_{t}\right) 1_{j, k}\left(X_{t}\right) ; f\left(X_{s}\right) 1_{j, k}\left(X_{s}\right)\right) \leq \\
& \left.8 \alpha^{1 / r} \tilde{H}^{p+q}\left|\frac{n_{j}}{N}+\frac{(\log N)^{1 / 2+1 / 2 \theta} \sigma_{\epsilon}}{\sqrt{N}}\left(\sqrt{N 2^{j_{1}}}+N^{\delta^{\prime}+1 / 2}\right)\right|^{\beta(q+p)} 2^{-j\left(1 / p^{*}+1 / q^{*}\right.}\right) .
\end{aligned}
$$

De (3.66) e (3.79) se tem

$$
\begin{aligned}
& \operatorname{Var}\left(\sum_{t=1}^{N} f\left(X_{t}\right) 1_{j+1,2 k}\left(X_{t}\right)\right) \leq \frac{n_{j}^{2}}{N n_{j}}\left|\frac{(\log N)^{1 / 2+1 / 2 \theta} \sigma_{\epsilon}}{\sqrt{N}}\left(\sqrt{N 2^{j_{1}}}+N^{\delta^{\prime}+1 / 2}\right)\right|^{2 \beta} \\
& +2 \frac{n_{j}^{2}}{N n_{j}}\left(8 \alpha^{1 / r} \tilde{H}^{p+q}\left|\frac{(\log N)^{1 / 2+1 / 2 \theta} \sigma_{\epsilon}}{\sqrt{N}}\left(\sqrt{N 2^{j_{1}}}+N^{\delta^{\prime}+1 / 2}\right)\right|^{\beta(q+p)} 2^{-j\left(1 / p^{*}+1 / q^{*}\right)}\right) \\
& =\frac{n_{j}}{N}\left|\frac{(\log N)^{1 / 2+1 / 2 \theta} \sigma_{\epsilon}}{\sqrt{N}}\left(\sqrt{N 2^{j_{1}}}+N^{\delta^{\prime}+1 / 2}\right)\right|^{2 \beta} \\
& +2 \frac{n_{j}}{N}\left(8 \alpha^{1 / r}\left|\frac{(\log N)^{1 / 2+1 / 2 \theta} \sigma_{\epsilon}}{\sqrt{N}}\left(\sqrt{N 2^{j_{1}}}+N^{\delta^{\prime}+1 / 2}\right)\right|^{\beta(q+p)} 2^{-j(1+1 / r}\right)
\end{aligned}
$$

\section{Proposição 3.3.2:}

$$
\begin{aligned}
E\left(\hat{s}_{j_{0}, k}-\hat{b}_{j_{0}, k}\right)^{2} & =\frac{1}{N^{2}} E\left[\sum_{t} \epsilon_{t} \phi_{j_{0}, k}\left(X_{t}\right)\right]^{2} \\
& =\frac{1}{N^{2}}\left(\operatorname{Var} \sum_{t=1}^{N} \epsilon_{t} \phi_{j_{0}, k}\left(X_{t}\right)\right)=\frac{\sigma_{\epsilon}^{2}}{N^{2}} \sum_{t=1}^{N} \operatorname{Var}\left(\phi_{j_{0}, k}\left(X_{t}\right)\right) \\
& +2 \sum_{t} \sum_{s \neq t} \operatorname{Cov}\left(\phi_{j_{0}, k}\left(X_{t}\right) ; \phi_{j_{0}, k}\left(X_{s}\right)\right)
\end{aligned}
$$

onde $\operatorname{Var}\left(\phi_{j_{0}, k}\left(X_{t}\right)\right)=2^{-j_{0}}\left(1-2^{-j_{0}}\right), \operatorname{logo}$

$$
\begin{aligned}
\frac{\sigma_{\epsilon}^{2}}{N^{2}} \sum_{t=1}^{N} \operatorname{Var}\left(\phi_{j_{0}, k}\left(X_{t}\right)\right) & =\frac{2^{j_{0}} \sigma_{\epsilon}^{2} n_{j}^{2}}{N n_{j}} 2^{-j_{0}}\left(1-2^{-j_{0}}\right) \\
& =\frac{\sigma_{\epsilon}^{2} n_{j}}{N}\left(1-2^{-j_{0}}\right)
\end{aligned}
$$


por outro lado, tem-se que

$$
\operatorname{Cov}\left(\phi_{j_{0}, k}\left(X_{t}\right) ; \phi_{j_{0}, k}\left(X_{s}\right)\right)=2^{-j_{0} / 2} \operatorname{Cov}\left(1_{j_{0}, k}\left(X_{t}\right) ; 1_{j_{0}, k}\left(X_{s}\right)\right)
$$

$\log 0$

$$
\begin{aligned}
\operatorname{Cov}\left(1_{j_{0}, k}\left(X_{t}\right) ; 1_{j_{0}, k}\left(X_{s}\right)\right) & \leq 8 \alpha^{1 / r}\left(E\left|1_{j_{0}, k}\left(X_{t}\right)\right|^{p}\right)^{1 / p}\left(E\left|1_{j_{0}, k}\left(X_{s}\right)\right|^{q}\right)^{1 / q} \\
& \leq 8 \alpha^{1 / r}\left(2^{-j_{0} / p}\right)\left(2^{-j_{0} / q}\right) .
\end{aligned}
$$

Segue-se que

$$
\frac{2^{j_{0}}}{N n_{j}} \sum_{t} \sum_{s \neq t} \operatorname{Cov}\left(\phi_{j_{0}, k}\left(X_{t}\right) ; \phi_{j_{0}, k}\left(X_{s}\right)\right) \leq \frac{2^{j_{0}}}{N} 2^{-j_{0} / 2} 8 \alpha^{1 / r} \frac{2^{-j_{o}(1 / p+1 / q)}}{N}
$$

e

$$
E\left(\hat{s}_{j_{0}, k}-\hat{b}_{j_{0}, k}\right)^{2} \leq \frac{\sigma_{\epsilon}^{2} n_{j}}{N}\left(1-2^{-j_{0}}\right)+\frac{8 \alpha^{1 / r}}{N} \frac{2^{-j_{o}(1 / p+1 / q-1 / 2)}}{N}
$$

\section{Lema 3.3.1:}

Seguindo o argumento da prova do lema 5.6.4 de Delouille (2001), tem-se que

$$
\begin{aligned}
E\left[\eta_{s}\left(\rho_{j k}, t\right)^{2}\right] & =E\left[\left(\left|\rho_{j k}\right|-t\right) 1_{\left|\rho_{j k}\right|>t}\right] \\
& =\int_{0}^{\infty} P\left[\left(\left|\rho_{j k}\right|-t\right) 1_{\left|\rho_{j k}\right|>t}>z\right] 2 z d z \\
& =\int_{0}^{\infty} P\left(\left(\left|\rho_{j k}\right|-t\right)>z\right) 2 z d z \\
& =\int_{0}^{\infty} P\left(\left|\rho_{j k}\right|>z+t\right) 2 z d z
\end{aligned}
$$

No caso particular da ondaleta de Haar, observa-se:

$$
\left|\rho_{j k}\right| \leq \frac{2^{j / 2}}{N}\left\{\left|\sum_{t} \epsilon_{t} 1_{j+1,2 k}\left(X_{t}\right)\right|+\left|\sum_{t} \epsilon_{t} 1_{j+1,2 k+1}\left(X_{t}\right)\right|\right\} .
$$

Seja $\mathcal{I}=I_{j=1, k}$; toda a análise é feita só para $(j+1,2 k)$, já que, de forma análoga, pode-se fazer para $(j+1,2 k+1)$. Sem perda de generalidade, muda-se $(j+1,2 k)$ por $(j, k)$. Como feito na demonstração do teorema 3.3.1, o intervalo aleatório $\mathcal{I}$ é aproximado pela união de intervalos fixos, começando pela partição de quantis teóricos como dado na expressão (3.11). 
Assim temos:

$$
\left|\sum_{t} \epsilon_{t} 1_{\mathcal{I}}\left(X_{t}\right)\right| \leq \sum_{t=1}^{d_{N}}\left|\epsilon_{t} 1_{\mathcal{I}}^{o}\left(X_{t}\right)\right|+\sum_{t=1}^{N}\left|\epsilon_{t}\right| 1_{I_{j^{*}, l_{1}}^{o} \cup I_{j^{*}, l_{2}}^{o}}\left(X_{t}\right)
$$

Seja $z^{\prime}=z+t, U_{j, k, l}=\frac{2^{j / 2}}{N}\left|\sum_{t} \epsilon_{t} 1_{\mathcal{I}_{j_{l}, k_{l}}^{o}}\left(X_{t}\right)\right|$ e $U_{j^{*}, m, d_{N}}=\frac{2^{j^{*} / 2}}{N} \sum_{t=1}^{N}\left|\epsilon_{t}\right| 1_{j^{*}, k}\left(X_{t}\right)$. Como mostrado em Delouille (2001),

$$
P\left(\left|\rho_{j k}\right|>z^{\prime}\right) \leq \sum_{l=1}^{d_{N}} P\left(U_{j, k, l} \geq z^{\prime} 2^{-l / \tilde{k}}\right)+\sum_{i=1,2} P\left(U_{j^{*} l_{i}, d_{N}} \geq z^{\prime} 2^{-d_{N} / \tilde{k}}\right)
$$

O objetivo é demonstrar a seguintes desigualdades:

$$
\begin{gathered}
\int_{0}^{\infty} P\left(U_{j, k, l} \geq z^{\prime} 2^{-l / \tilde{k}}\right) \leq C \frac{(\log N)^{1 / r+1 / \theta}}{N^{1-\alpha}} \\
\int_{0}^{\infty} P\left(U_{j^{*} l_{i}, d_{N}} \geq z^{\prime} 2^{-d_{N} / \tilde{k}}\right) \leq \frac{(\log N)^{1 / \theta+1 / 2 r}}{N^{1-2 \delta+\alpha}} .
\end{gathered}
$$

Temos as seguintes desigualdades quando $x \leq 3 \operatorname{Var}\left(U_{t}\right) / M$; que é a parte central da distribuição,

$$
P\left(\left|\sum_{t} Z_{t}\right| \geq \lambda\right) \leq 4 \exp \left\{\frac{-N \lambda^{2}}{8 m M E\left[U_{t}^{2}\right]}\right\}
$$

Agora, para as caudas da distribuição é satisfeita a desigualdade $x \geq 3 \operatorname{Var}\left(U_{t}\right) / M$ e

$$
P\left(\left|\sum_{t} Z_{t}\right| \geq \lambda\right) \leq 4 \exp \left\{\frac{-3 N \lambda}{8 m M}\right\}
$$

Primeiro vamos analisar o caso quando $x \leq 3 \operatorname{Var}\left(U_{t}\right) / M$. Nesta situação tem-se que $x=$ $z^{\prime} 2^{-l / \tilde{k}} N 2^{-j / 2}$, o que significa que

$$
z^{\prime}=z+t \leq 3 \operatorname{Var}\left(U_{t}\right) 2^{l / \tilde{k}} N 2^{j / 2} M^{-1}=: a_{1} \quad \text { e } \quad z \leq a_{1}-t=: b
$$

logo a integral $\int_{0}^{\infty} P\left(U_{j, k, l} \geq z^{\prime} 2^{-l / \tilde{k}}\right) 2 z d z$ pode-se decompor, como segue:

$$
\begin{aligned}
\int_{0}^{\infty} P\left(U_{j, k, l} \geq z^{\prime} 2^{-l / \tilde{k}}\right) 2 z d z & =\int_{0}^{b} P\left(U_{j, k, l} \geq z^{\prime} 2^{-l / \tilde{k}}\right) 2 z d z \\
& +\int_{b}^{\infty} P\left(U_{j, k, l} \geq z^{\prime} 2^{-l / \tilde{k}}\right) 2 z d z
\end{aligned}
$$


ao usar a desigualdade (3.93) e denotar por $a_{2}=: 2^{-l / \tilde{k}} N 2^{-j / 2}$, trabalhando com o lado direito da igualdade (3.96), obtém-se:

$$
\int_{0}^{b} P\left(U_{j, k, l} \geq(z+t) a_{2}\right) 2 z d z \leq \int_{0}^{b} 4 \exp \left\{\frac{-N\left((z+t) a_{2}\right)^{2}}{8 M m E\left[U_{t}^{2}\right]}\right\} 2 z d z+4 \gamma \frac{N}{m} \exp \left\{-c m^{\theta}\right\}
$$

Seja $u=(z+t) /\left(E\left[U_{t}^{2}\right]\right)^{1 / 2}, \operatorname{logo}(3.98)$ torna-se:

$$
\begin{aligned}
& \int_{t /\left(E\left[U_{t}^{2}\right]\right)^{1 / 2}}^{a_{1}} 4 \exp \left\{\frac{-N\left(u a_{2}\right)^{2}}{64 M m}\right\}(u-t) d u+4 \gamma \frac{N}{m} \exp \left\{-c m^{\theta}\right\} \\
\leq & \int_{t /\left(E\left[U_{t}^{2}\right]\right)^{1 / 2}}^{\infty} 4 \exp \left\{\frac{-N\left(u a_{2}\right)^{2}}{64 M m}\right\} u d u+4 \gamma \frac{N}{m} \exp \left\{-c m^{\theta}\right\} \\
- & t \int_{t /\left(E\left[U_{t}^{2}\right]\right)^{1 / 2}}^{\infty} 4 \exp \left\{\frac{-N\left(u a_{2}\right)^{2}}{64 M m}\right\} d u-4 \gamma \frac{N}{m} \exp \left\{-c m^{\theta}\right\} .
\end{aligned}
$$

A integral (3.101) pode ser desprezada, por ter sinal negativo. Agora vamos analisar a integral (3.100), que é igual a

$$
\begin{aligned}
& \int_{t /\left(E\left[U_{t}^{2}\right]\right)^{1 / 2}}^{\infty} 4 \exp \left\{\frac{-N\left(u a_{2}\right)^{2}}{64 M m}\right\} u d u \\
= & \int_{t /\left(E\left[U_{t}^{2}\right]\right)^{1 / 2}}^{\infty}\left[\exp \left\{\frac{-N a_{2}^{2}}{64 m M} u^{2}\right\} \frac{-64 M m}{N a_{2}^{2}}\right] d u \\
= & 8\left(\frac{64 M m}{N a_{2}^{2}}\right) \exp \left\{\frac{-t^{2} a_{2}^{2} N}{E\left[U_{t}^{2}\right] 64 M m}\right\},
\end{aligned}
$$

logo, substituindo o valor de t pelo valor dado na expressão (3.38) e $a_{2}$ conclui-se

$$
\begin{aligned}
& 8\left(\frac{64 M m}{N\left(2^{-2 l / \tilde{k}} N^{2} 2^{-j}\right)}\right) \exp \left\{\frac{-\left(N^{-1} \sigma_{\epsilon}^{2} 2^{-j_{1}}(\log N)^{1+1 / \theta}\right)\left(2^{-2 l / \tilde{k}} N^{2} 2^{-j}\right) N}{E\left[U_{t}^{2}\right] 64 M m}\right\} \\
= & 8\left(\frac{64 M(\log N)^{1 / \theta}}{\left(2^{-2 l / \tilde{k}} N^{3} 2^{-j}\right)}\right) \exp \left\{\frac{-\left(\sigma_{\epsilon}^{2} 2^{-j_{1}}(\log N)\right)\left(2^{-2 l / \tilde{k}} N^{2} 2^{-j}\right)}{E\left[U_{t}^{2}\right] 64 M}\right\} .
\end{aligned}
$$

Temos que o termo $2^{d_{N} / \tilde{k}}=o(\log N)^{1 / r}, \operatorname{com} r>1,2^{j}=o\left(N^{1-\alpha}\right)$ para $j \in \mathcal{J}$ e $M=C N$, de onde pode-se concluir que $\frac{64 M(\log N)^{1 / \theta}}{\left(2^{-2 l / \tilde{k}} N^{3} 2^{-j}\right)}$ é da ordem $o\left(\frac{(\log N)^{1 / r+1 / \theta}}{N^{1+\alpha}}\right) \operatorname{com} 0<\alpha<1$. Agora vejamos a ordem de convergência da exponencial. Sabe-se que $E\left[U_{t}^{2}\right] \leq N(\log N) 2^{-j_{i}}, 2^{j_{i}-j}$ é $o(1)$, logo o termo exponencial tem ordem de convergência $o(1)$. 
Para a convergência da integral (3.97) obtemos

$$
\begin{aligned}
& \int_{b}^{\infty} P\left(\left|\sum_{t} Z_{t}\right|>(z+t) 2^{-l / \tilde{k}} N 2^{-j / 2}\right) 2 z d z \\
\leq & \int_{b}^{\infty} 4 \exp \left\{\frac{-3 N(z+t) a_{2}}{8 M m}\right\} 2 z d z=\int_{b}^{\infty} 8 \exp \left\{\frac{-3 N u a_{2}}{8 M m}\right\} u d u \\
- & t \int_{b}^{\infty} 4 \exp \left\{\frac{-3 N u a_{2}}{8 M m}\right\} d u=A+B .
\end{aligned}
$$

A integral B na expressão (3.108) é desprezada pelo seu sinal negativo, então vamos estudar o termo A.

$$
\int_{b}^{\infty} 8 \exp \left\{\frac{-3 N u a_{2}}{8 M m}\right\} u d u=\exp \left\{\frac{-3 N a_{2} a_{1}}{8 M m}\right\}\left(\frac{a_{1} 8 M m}{3 N a_{2}}+\frac{64 m^{2} M^{2}}{9 N a_{2}^{2}}\right)
$$

simplificando os produtos de termos da forma

$$
\begin{aligned}
a_{2} a_{1} & =\left(2^{-l / \tilde{k}} N 2^{-j / 2}\right)\left(3 E\left(U_{t}^{2}\right) 2^{l / \tilde{k}} N^{-1} 2^{j / 2} M^{-1}\right) \\
& =3 M^{-1}\left(E\left[U_{t}^{2}\right]\right)^{2}
\end{aligned}
$$

$\mathrm{e}$

$$
\frac{a_{1}}{a_{2}}=\frac{3 E\left(U_{t}^{2}\right) 2^{l / \tilde{k}} N^{-1} 2^{j / 2} M^{-1}}{2^{-l / \tilde{k}} N 2^{-j / 2} E\left(U_{t}^{2}\right)}=\frac{32^{2 l / \tilde{k}} 2^{j}}{M N^{2}}
$$

substituindo (3.110) e (3.111) em (3.109) obtemos

$$
\begin{aligned}
\frac{-3 N a_{2} a_{1}}{8 M m} & =\frac{-9 N E\left[U_{t}^{2}\right]}{8 M^{2} m} \\
\exp \left\{\frac{-9 N E\left[U_{t}^{2}\right]}{8 M^{2} m}\right\} & =o(1) \\
\frac{a_{1} 8 M m}{3 N a_{2}} & =\frac{82^{2 l / \tilde{k}} 2^{j} m}{N^{3}}=\frac{8(\log N)^{1 / r+1 / \theta} N^{1-\alpha}}{N^{3}} \\
& =o\left(\frac{(\log N)^{1 / r+1 / \theta}}{N^{2+\alpha}}\right) \\
\frac{64 m^{2} M^{2}}{9 N a_{2}^{2}} & =\frac{64 m^{2} M^{2} 2^{-l / \tilde{k}} 2^{j}}{9 N^{3} E\left[U_{t}^{2}\right]}=\frac{64(\log N)^{1 / r+1 / 2 \theta} N^{3-\alpha}}{9 N^{4}(\log N)^{1 / \theta}} \\
& =o\left(\frac{(\log N)^{1 / r+1 / \theta}}{N^{1-\alpha}}\right),
\end{aligned}
$$


Resumindo temos as seguintes desigualdades:

$$
\begin{gathered}
\int_{0}^{b} P\left(U_{j, k, l} \geq z^{\prime} 2^{-l / \tilde{k}}\right) 2 z d z \leq C \frac{(\log N)^{1 / \theta+1 / r}}{N^{1+\alpha}} \\
\int_{b}^{\infty} P\left(U_{j, k, l} \geq z^{\prime} 2^{-l / \tilde{k}}\right) 2 z d z \leq C \frac{(\log N)^{1 / r+1 / \theta}}{N^{1-\alpha}}
\end{gathered}
$$

e (3.91) fica provado.

Agora vamos ver a ordem de convergência de

$$
\begin{gathered}
\int_{0}^{\infty} P\left(U_{j, l_{i}, d_{N}}>z^{\prime} 2^{-d_{N} / \tilde{k}}\right) 2 z d z \quad \text { com } i=1,2 \\
P\left(U_{j, l_{i}, d_{N}}>z^{\prime} 2^{-d_{N} / \tilde{k}}\right)=P\left(\frac{2^{j / 2}}{N} \sum_{t=1}^{N}\left|\epsilon_{t}\right| 1_{j^{*}, l_{i}}^{0}\left(X_{t}\right) \geq(z+t) 2^{-d_{N} / \tilde{k}}\right) .
\end{gathered}
$$

Sabe-se que $\sup _{t}\left|\epsilon_{t}\right| \leq N^{\delta}$. Seja $W_{t}=1_{j^{*}, l_{i}}^{0}\left(X_{t}\right)-2^{j^{*}}$, com média zero e $\operatorname{Var}\left(W_{t}\right)=2^{-j^{*}}\left(1-2^{-j^{*}}\right)$. Então,

$$
\begin{aligned}
& P\left(\sum_{t=1}^{N} 1_{j^{*}, l_{i}}^{0}\left(X_{t}\right) \geq(z+t) 2^{-d_{N} / \tilde{k} N 2^{-j / 2}} N^{-\delta}\right) \\
& =P\left(\sum_{t=1}^{N} 1_{j^{*}, l_{i}}^{0}\left(X_{t}\right)-2^{-j^{*}} \geq(z+t) 2^{-d_{N} / \tilde{k} N 2^{-j / 2}} N^{-\delta}-N 2^{-j^{*}}\right) \\
& =P\left(\sum_{t=1}^{N} W_{t}>\lambda\right)
\end{aligned}
$$

com $\lambda=(z+t) 2^{-d_{N} / \tilde{k} N 2^{-j / 2}} N^{-\delta}-N 2^{-j^{*}}$. De forma análoga como foi provado para a integral na primeira parte, os lemas 3.5.1 e 3.5.1 são usados para introduzir os blocos independentes de comprimento $c_{\lambda} \log N$, que com uma probabilidade alta, têm a mesma distribuição dos blocos originais de dados. Segue-se que se $\lambda<3 \operatorname{Var}\left(U_{t}\right) M^{-1}$, isto é $(z+t) 2^{-d_{N} / \tilde{k}} N 2^{-j / 2} N^{-\delta}-N 2^{-j^{*}}<$ $3 \operatorname{Var}\left(U_{t}\right) M^{-1}$, então $(z+t)<\tilde{a}_{1}=:\left(3 \operatorname{Var}\left(U_{t}\right) M^{-1}+N 2^{-j^{*}}\right) 2^{d_{N} / \tilde{k}} N^{-1} 2^{j / 2} N^{\delta}$. Agora vamos 
decompor a integral (3.118) em duas partes.

$$
\begin{aligned}
& \int_{0}^{\infty} P\left(U_{j, l_{i}, d_{N}}>z^{\prime} 2^{-d_{N} / \tilde{k}}\right) 2 z d z \\
= & \int_{0}^{\tilde{a}_{1}-t} P\left(\left|\sum_{t=1}^{N} W_{t}\right|>(z+t) 2^{-d_{N} / \tilde{k}} N 2^{-j / 2} N^{-\delta}-N 2^{-j^{*}}\right) 2 z d z \\
+ & \int_{\tilde{a}_{1}-t}^{\infty} P\left(\left|\sum_{t=1}^{N} W_{t}\right|>(z+t) 2^{-d_{N} / \tilde{k}} N 2^{-j / 2} N^{-\delta}-N 2^{-j^{*}}\right) 2 z d z \\
& \int_{0}^{\tilde{a}_{1}-t} P\left(\left|\sum_{t=1}^{N} W_{t}\right|>(z+t) 2^{-d_{N} / \tilde{k}} N 2^{-j / 2} N^{-\delta}-N 2^{-j^{*}}\right) 2 z d z \\
\leq & \int_{0}^{\tilde{a}_{1}-t} 4 \exp \left\{\frac{-N\left((z+t) a_{2}-N 2^{-j^{*}}\right)^{2}}{8 m M E\left[U_{t}^{2}\right]}\right\}
\end{aligned}
$$

onde $a_{2}=:\left(2^{-d_{N} / \tilde{k}} N 2^{-j / 2} N^{-\delta}\right)$ e seja $u=\frac{(z+t) \tilde{a}_{2}-N 2^{-j^{*}}}{E\left[U_{t}^{2}\right]}$. Substituindo em (3.121) tem-se

$$
\begin{aligned}
& \int_{\left(t \tilde{a}_{2}-N 2^{-j^{*}}\right) / E\left[U_{t}^{2}\right]}^{\infty} 8 \exp \left\{\frac{-N u^{2}}{8 M m}\right\}\left(\frac{u E\left[U_{t}^{2}\right]+N 2^{-j^{*}}}{\tilde{a}_{2}}-t\right) d u \\
= & \int_{\left(t \tilde{a}_{2}-N 2^{-j^{*}}\right) / E\left[U_{t}^{2}\right]}^{\infty} \operatorname{sexp}\left\{\frac{-N u^{2}}{8 M m}\right\}\left(\frac{u E\left[U_{t}^{2}\right]}{\tilde{a}_{2}}\right) d u \\
+ & \int_{\left(t \tilde{a}_{2}-N 2^{-j^{*}}\right) / E\left[U_{t}^{2}\right.}^{\infty} 8 \exp \left\{\frac{-N u^{2}}{8 M m}\right\}\left(\frac{N 2^{-j^{*}}}{\tilde{a}_{2}}-t\right) d u .
\end{aligned}
$$

Desta forma, concluímos que:

$$
\begin{aligned}
& \int_{\left(t \tilde{a}_{2}-N 2^{-j^{*}}\right) / E\left[U_{t}^{2}\right]}^{\infty} 8 \exp \left\{\frac{\left.-N u^{2}\right\}}{8 M m}\right\}\left(\frac{u E\left[U_{t}^{2}\right]}{\tilde{a}_{2}}\right) d u \\
= & \frac{8 E\left[U_{t}^{2}\right]}{\tilde{a}_{2}} \int_{\left(t \tilde{a}_{2}-N 2^{-j^{*}}\right) / E\left[U_{t}^{2}\right]}^{\infty}\left[\exp \left\{\frac{-N u^{2}}{64 M m}\right\}\left(\frac{64 M m}{N}\right)\right]^{\prime} d u \\
= & \frac{64 M m E\left[U_{t}^{2}\right]}{N \tilde{a}_{2}} \exp \left\{\frac{-N\left(t \tilde{a}_{2}-N 2^{-j^{*}}\right)}{64 M m E\left[U_{t}^{2}\right]}\right\}, \\
t \tilde{a}_{2} & =\left(N^{\delta^{\prime}}\left(4 N^{-1} \lambda s(\log (N))^{1+1 / \theta} \sigma_{W^{*}}^{2}\right)^{1 / 2}+N 2^{-j^{*}}\right)\left(2^{-d_{N} / \tilde{k}} N 2^{-j / 2} N^{-\delta}\right) \\
& =N^{\delta^{\prime}+1 / 2-\delta}(\log N)^{1 / 2+1 / 2 \theta} 2^{-d_{N} / \tilde{k}} 2^{-j / 2} \sigma_{w}^{*}+N^{2+\delta-\delta^{\prime}} 2^{-j^{*}} 2^{-d_{N} / \tilde{k}_{2}} 2^{-j / 2}(3.125) \\
\left(t \tilde{a}_{2}-N 2^{j^{*}}\right) & =N^{\delta^{\prime}+1 / 2-\delta}(\log N)^{1 / 2+1 / 2 \theta} 2^{-d_{N} / \tilde{k}} 2^{-j / 2} \sigma_{w} \\
& -N 2^{j^{*}}\left(N^{1+\delta-\delta^{\prime}} 2^{-d_{N} / \tilde{k}} 2^{-j / 2}-1\right),
\end{aligned}
$$




$$
\begin{aligned}
& \frac{-N\left(t \tilde{a}_{2}-N 2^{j^{*}}\right)}{64 M m E\left[U_{t}^{2}\right]}=\frac{-N^{\delta^{\prime}+3 / 2-\delta}(\log N)^{1 / 2+1 / 2 \theta} 2^{-d_{N} / \tilde{k}} 2^{-j / 2} \sigma_{w}}{64 M m(\log N) N 2^{-j^{*}}} \\
+ & \frac{N^{2} 2^{-j^{*}}\left(N^{1+\delta-\delta^{\prime}} 2^{-d_{N} / \tilde{k}} 2^{-j / 2}-1\right)}{64 M m(\log N) N 2^{-j^{*}}} \\
= & \frac{-N^{\delta-\delta^{\prime}+3 / 2}(\log N)^{1 / \theta-1 / r-1 / 2} 2^{-j / 2} 2^{-j^{*} / 2} N \sigma_{\epsilon}}{64 K N^{2}} \\
+ & \frac{N^{2} 2^{-j^{*}}\left(N^{1+\delta-\delta^{\prime}}(\log N)^{-1 / r} N^{-1 / 2-\alpha / 2}\right)}{64 k N(\log N)^{1 / \theta+1} N 2^{-j^{*}}}-\frac{1}{64 k(\log N)^{1 / \theta+1}} \\
= & \frac{-(\log N)^{1 / \theta-1 / r-1 / 2}}{N^{\delta-\delta^{\prime}-1 / 2}}+\frac{N^{1 / 2+\alpha / 2+\delta-\delta^{\prime}}(\log N)^{-1-1 / r-1 / \theta}}{64 k} \\
\frac{64 M m}{N \tilde{a}_{2}}= & \frac{64 M m E\left[U_{t}^{2}\right]}{N^{2-\delta} 2^{-d_{N} / \tilde{k}} 2^{-j / 2}}=\frac{64 k N(\log N)^{1+1 / \theta} N 2^{j^{*}}}{N^{2-\delta} 2^{-d_{N} / \tilde{k}} 2^{j / 2}} \\
= & o\left(\frac{(\log N)^{1 / \theta+1 / r+1}}{N^{1 / 2-\delta-\alpha / 2}}\right)
\end{aligned}
$$

Assim o termo exponencial tem uma ordem muito menor que o termo $\frac{64 M m E\left[U_{t}^{2}\right]}{N \tilde{a}_{2}}, \operatorname{logo}$

$$
\int_{\left(t \tilde{a}_{2}-N 2^{-j^{*}}\right) / E\left[U_{t}^{2}\right]}^{\infty} 8 \exp \left\{\frac{-N u^{2}}{8 M m}\right\}\left(\frac{u E\left[U_{t}^{2}\right]}{\tilde{a}_{2}}\right) d u \leq C\left(\frac{(\log N)^{1 / \theta+1 / r+1}}{N^{1 / 2-\delta-\alpha / 2}}\right) .
$$

A seguir vamos ver a convergência da outra integral $\int_{t \tilde{a}_{2}-N 2^{-j^{*}} E\left[U_{t}^{2}\right]}^{\infty} 8 \exp \left\{\frac{-N u^{2}}{8 M m}\right\}\left(\frac{N 2^{-j^{*}}}{\tilde{a}_{2}}-t\right) d u$. Como mostrado em Delouille (2001), é assintoticamente negativa. Mostraremos agora a taxa de convergência para $\int_{\tilde{a}_{1}-t}^{\infty} P\left(\left|\sum_{t=1}^{N} W_{t}\right|>(z+t) 2^{-d_{n} / \tilde{k}} N 2^{-j / 2} N^{-\delta}-N 2^{-j^{*}}\right) 2 z d z$. Se $\lambda=$ $(z+t) 2^{-d_{N} / \tilde{k} N 2^{-j / 2}} N^{-\delta}-N 2^{-j^{*}}$ for maior que $3 E\left[U_{t}^{2}\right] M^{-1}$, então a integral pode-se limitar por

$$
\begin{aligned}
& \int_{\tilde{a}_{1}-t}^{\infty} 4 \exp \left\{\frac{-3 N\left((z+t) 2^{-d_{N} / \tilde{k}} N 2^{-j / 2} N^{-\delta}-N 2^{-j^{*}}\right)}{8 M m}\right\} 2 z d z \\
= & \int_{\tilde{a}_{1}}^{\infty} 4 \exp \left\{\frac{-3 N\left(u 2^{-d_{N} / \tilde{k}} N 2^{-j / 2} N^{-\delta}\right)}{8 M m}\right\} \exp \left\{\frac{N 2^{-j^{*}}}{8 M m}\right\} u d u \\
- & t \int_{\tilde{a}_{1}}^{\infty} 4 \exp \left\{\frac{-3 N\left(u 2^{-d_{N} / \tilde{k}} N 2^{-j / 2} N^{-\delta}\right)}{8 M m}\right\} \exp \left\{\frac{N 2^{-j^{*}}}{8 M m}\right\} d u .
\end{aligned}
$$

A integral (3.131) é negativa, portanto não é levada em conta. Para (3.130), seja $\tilde{a}_{4}=$ : 
$\frac{3 N 2^{-d_{N} / \tilde{k}} N 2^{-j / 2}}{8 N^{\delta} M m}, \log \mathrm{O}$

$$
\begin{aligned}
\exp \left\{\frac{N 2^{-j^{*}}}{8 M m}\right\} \int_{\tilde{a}_{1}}^{\infty} 4 \exp \left\{u \tilde{a}_{4}\right\} u d u & =\exp \left\{\frac{N 2^{-j^{*}}}{8 M m}\right\} \frac{\exp \left\{-\tilde{a}_{4} \tilde{a}_{1}\right\}}{\tilde{a}_{4}}\left(\tilde{a}_{1}+\tilde{a}_{4}^{-1}\right), \\
\tilde{a}_{1} \tilde{a}_{4} & =\left(\left(3 E\left[U_{t}^{2}\right] M^{-1}+N 2^{-j^{*}}\right) 2^{d_{N} / \tilde{k}} N^{-1} 2^{j / 2} N^{\delta}\right)\left(\frac{3 N 2^{-d_{N} / \tilde{k}} N 2^{-j / 2}}{8 N^{\delta} M m}\right) \\
& =\frac{9 N E\left[U_{t}^{2}\right]}{8 m}+\frac{N 2^{-j^{*}} 3 N^{2}}{8 N^{\delta} M m}=\frac{9(\log N) 1-1 / \theta}{8 k}+\frac{3(\log N)^{1 / \theta}}{8 k},(3.133) \\
\tilde{a}_{1} \tilde{a}_{4}^{-1} & =\frac{\left(\left(3 E\left[U_{t}^{2}\right] M^{-1}+N 2^{-j^{*}}\right) 2^{d_{N} / \tilde{k}} N^{-1} 2^{j / 2} N^{\delta}\right)\left(8 N^{\delta} M m\right)}{3 N 2^{-d_{N} / \tilde{k}} N 2^{-j / 2}} \\
& =\frac{24 E\left[U_{t}^{2}\right] N^{2 \delta}-12^{2 d_{N} / \tilde{k}} 2^{j} m}{3 N^{2}}+\frac{N 2^{-j^{*}} 2^{d_{N} / \tilde{k}} 2^{j} N^{2 \delta-1} M m}{3 N^{2}} \\
& =\frac{(\log N)^{1+1 / \theta+1 / 2 r} N 2^{-j^{*}} N^{2 \delta-\alpha}}{3 N^{2}}+\frac{(\log N)^{1 / 2 r+1 / \theta} N^{2 \delta-\alpha}}{3 N} \\
\tilde{a}_{4}^{-2} & =\frac{64 M^{2} m^{2}}{9 N^{2} 2^{2 d_{N} / \tilde{k}} 2^{j} N^{2-2 \delta}}=\frac{64 k(\log N)^{1 / 2 \theta-1 / 2 r}}{9 N^{2-2 \delta}} \leq C \frac{(\log N)^{1 / 2 \theta-1 / 2 r}}{N^{2-2 \delta}}(3.135)
\end{aligned}
$$

Assim, $\exp \left\{-\tilde{a}_{4} \tilde{a}_{1}\right\} \leq \exp \left\{-c(\log N)^{1-1 / \theta}\right\}, \exp \left\{\frac{N 2^{-j^{*}}}{8 M m}\right\}=o(1)$, portanto

$$
\exp \left\{\frac{N 2^{-j^{*}}}{8 M m}\right\} \frac{\exp \left\{-\tilde{a}_{4} \tilde{a}_{1}\right\}}{\tilde{a}_{4}}\left(\tilde{a}_{1}+\tilde{a}_{4}^{-1}\right)<C \frac{(\log N)^{1 / \theta+1 / 2 r}}{N^{1-2 \delta+\alpha}}
$$

Segue-se, finalmente, que

$$
\int_{\left(t \tilde{a}_{2}-N 2^{-j^{*}}\right) / E\left[U_{t}^{2}\right.}^{\infty} 8 \exp \left\{\frac{-N u^{2}}{8 M m}\right\}\left(\frac{N 2^{-j^{*}}}{\tilde{a}_{2}}-t\right) d u \leq C \frac{(\log N)^{1 / \theta+1 / 2 r}}{N^{1-2 \delta+\alpha}}
$$

e (3.92) fica provada. 



\section{Capítulo 4}

\section{Aplicações}

\subsection{Introdução}

Para ilustrar a utilidade dos modelos propostos neste trabalho são apresentadas a seguir algumas aplicações a dados reais.

\subsection{Aplicação 1}

De acordo com o modelo CAPM, espera-se que o risco sistemático de um ativo possa ser mensurado pela sua sensibilidade aos movimentos do mercado e, para isso, supõe-se que os retornos dos títulos são linearmente correlacionados com as flutuações de um índice de mercado. No entanto, podem existir relações não lineares entre o retorno de um ativo específico e as flutuações do índice de mercado. Para mais detalhes consultar Pereira (2010).

Nesta aplicação é estudada a relação entre a variação do preço da ação ARCZ6 (Aracruz Celulose S.A.) e a variação do índice Ibovespa. Os dados foram obtidos dos sites http://www.cedrofinances.com.br (ARCZ6) e http://www.bmfbovespa.com.br/indices (Ibovespa). Consistem de 103 observações mensais, referentes ao período compreendido entre maio de 2001 e novembro de 2009. Com o preço das ações e dos pontos do Ibovespa, calcula-se o retorno simples mensal do mês t, dado pela expressão

$$
R_{t}=\frac{P_{t}-P_{t-1}}{P_{t-1}}
$$

Na Figura 4.1 é apresentado o gráfico de dispersão entre os retornos do IBOVESPA e da ARCZ6, onde é possível observar menor concentração de pontos nos valores negativos do IBOVESPA. Na Figura 4.2, (a) e (b), são apresentados os gráficos dos retornos do IBOVESPA e das ações 


\section{Gráfico de Dispersão}

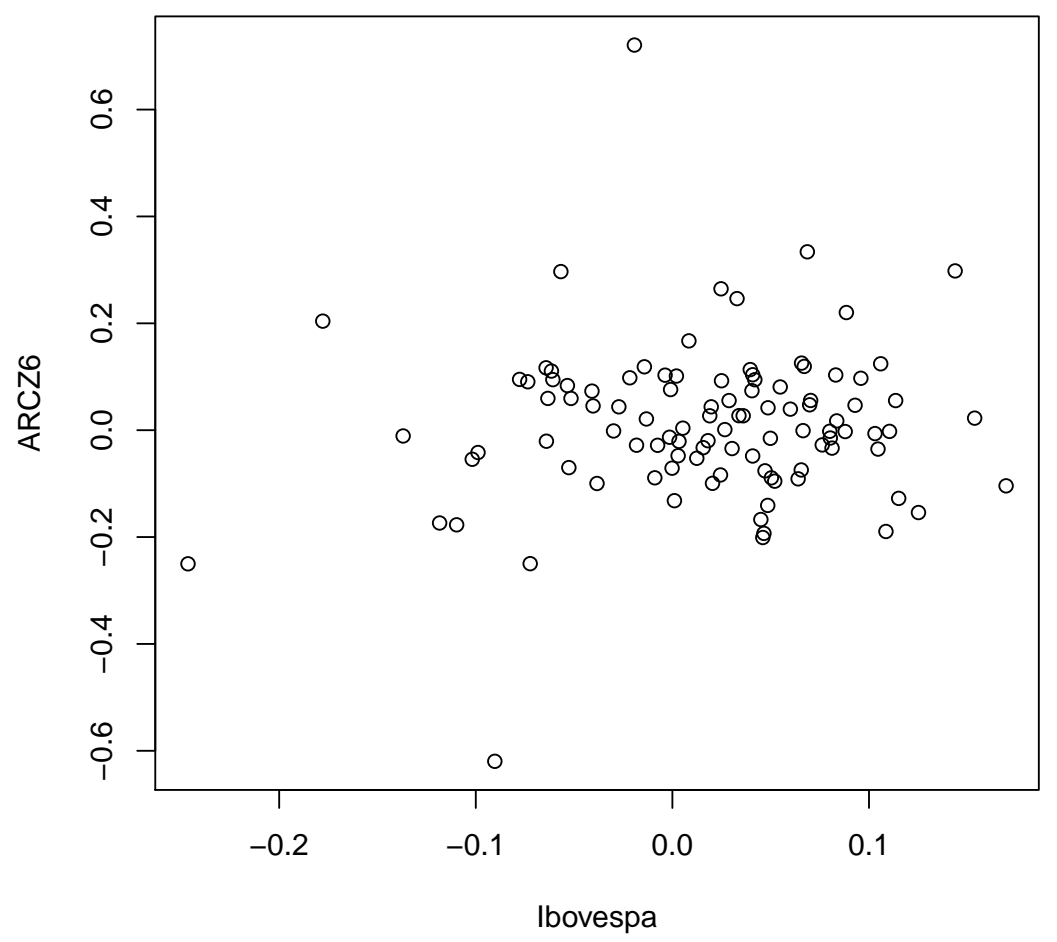

Figura 4.1: Gráfico de dispersão dos retornos mensais das ações ARCZ6 pelos retornos mensais do Índice Ibovespa

ARCZ6, respectivamente. Os retornos da ARCZ6 no último ano apresentam um aumento na variabilidade. Pelas acf amostral e pacf amostral dos retornos de IBOVESPA apresentadas na figuras (c) e (d), observa-se que a série tem um comportamento estacionário e um modelo AR(1) podia ser indicado para ajustá-la. As acf amostral e pacf amostral dos retornos de ARCZ6, mostradas na figuras (e) e (f), respectivamente, indicam que a série apresenta comportamento estacionário e um modelo $\mathrm{AR}(6)$ pode ser adequado para ajustar os dados.

Assim, vemos que as duas séries apresentam comportamento estacionário, satisfazendo as suposições do modelo para aplicar as metodologias de regressão não linear estudadas neste trabalho. 
(a)

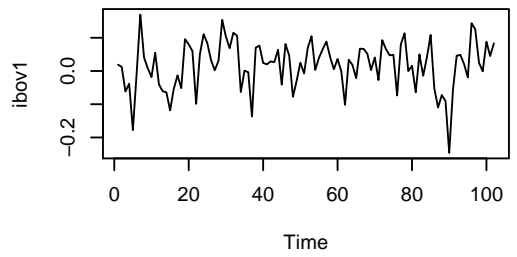

(c)

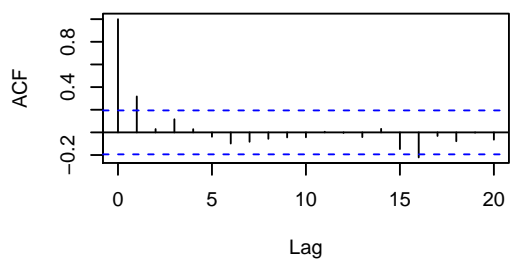

(e)

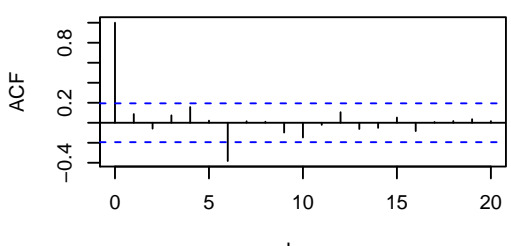

Lag (b)

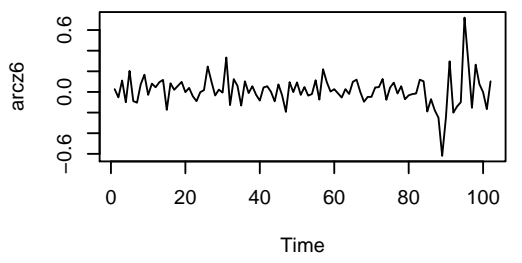

(d)

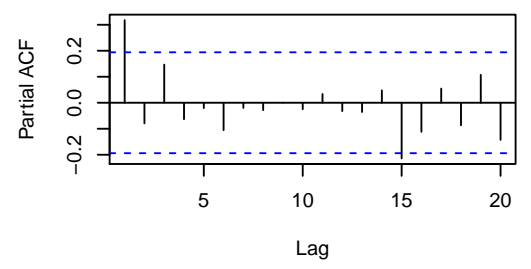

(f)

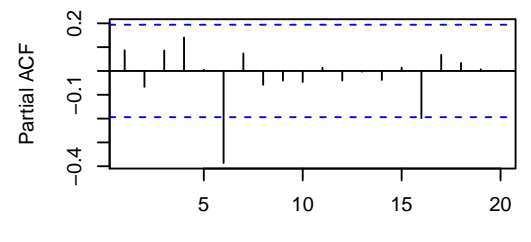

Lag

Figura 4.2: Gráficos de: (a) retornos mensais do IBOVESPA, entre maio de 2001 e novembro de 2009, (b) retornos mensais do ARCZ6 entre maio de 2001 e novembro de 2009, (c) função de autocorrelação amostral dos retornos do IBOVESPA, (d) função de autocorrelação parcial amostral dos retornos do IBOVESPA (e) função de autocorrelação amostral dos retornos da ARCZ6, (f) função de autocorrelação parcial amostral dos retornos da ARCZ6

$$
y_{t}=f\left(X_{t}\right)+\epsilon_{t}, \quad t=1, \ldots, N
$$

em que, $y_{t}$ é a variável retornos mensais do IBOVESPA e $X_{t}$ a variável retornos da ARCZ6.

A seguir serão ajustados os modelos de regressão não paramétrica usando a metodologia de ondaleta deformada com limiar duro. Primeiro será feita uma análise dos valores do RMSE e dos resíduos para escolher o valor de $\mathcal{K}$, como dado na expressão (2.19), e a base mais apropri- 
ada para a análise dos dados. Foram explorados valores de $\mathcal{K}=0,6 ; 0,7 ; 0,8 ; 0,9 ; 1 ; 1,1 ; 1,2 ;$ e 1,3, com as bases Symmlet6, Symmlet7, Symmlet8, Symmlet9, Symmlet10, Coiflet2, Coiflet3, Coiflet4 e Coiflet5.

$\mathrm{Na}$ análise exploratória, para valores de $\mathcal{K}=0,5 ; \ldots ; 0,8$, os valores do RMSE são maiores do que quando comparados aos outros valores de k. A Figura A.9 mostra as estimativas para o caso particular de $\mathcal{K}=0,5$. Os resíduos apresentam alta correlação serial, como mostra a Figura A.10 no Apêndice A. Para os valores de $\mathcal{K}=0,9, \ldots, 1,3$, as curvas ajustadas com as bases deformadas Symmlet6, Coiflet3 e Coiflet4 apresentam comportamento mais suave e seus resíduos, por sua vez, apresentam um comportamento de ruído branco com a base de ondaleta deformada Symmlet6. As Figuras A.11 e A.12, no Apêndice A, mostram as estimativas e os resíduos para as diferentes bases de ondaleta deformada com $\mathcal{K}=1,2$. De forma geral, a base de ondaleta Symmlet6 tem melhor ajuste (porque apresenta os valores de RMSE menores). Com as análises feitas, foi selecionado o valor de $\mathcal{K}=1,2$ e a base de ondaleta Symmlet6 para fazer o estudo específico de ajuste do modelo de regressão não paramétrica.

Na Figura 4.3, a linha contínua representa a estimativa obtida pelas ondaletas deformadas com

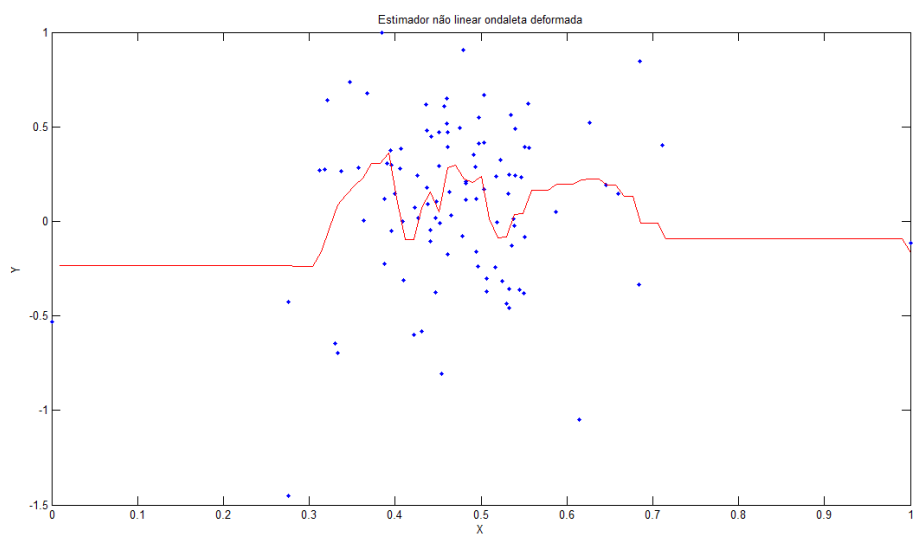

Figura 4.3: Os pontos representam o gráfico de dispersão dos retornos das ações de ARCZ6 pelas flutuações do Ibovespa e a linha sólida representa a $\hat{\beta}$ obtida com ondaletas deformadas com base Symmlet6 e valor $\mathcal{K}=1,2$.

base Symmlet6 e $\mathcal{K}=1,2$. A curva evidencia um comportamento não linear, onde a parte central é menos suave. Na Figura 4.4 (a), os resíduos apresentam um comportamento estacionário, fato que é confirmado pelos gráficos (c) e (d) da Figura 4.4. As funções de autocorrelação 

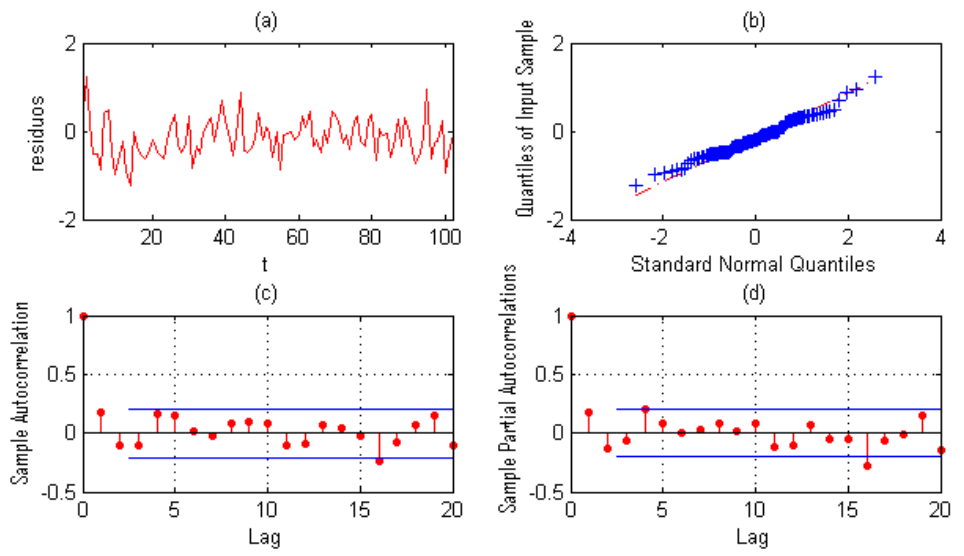

Figura 4.4: Gráficos dos resíduos obtido com ondaletas deformadas e base Symmlet6: (a) gráfico dos resíduos, (b)gráfico de quantis, (c)função de autocorrelação amostral, (d) função de autocorrelação parcial amostral.

amostral e autocorrelação parcial amostral, respectivamente, mostram um comportamento de ruído branco dos resíduos. A Figura 4.4 (b) apresenta o gráfico de quantis e não indica grandes desvios da hipótese de normalidade, apesar de sugerir caudas um pouca mais pesadas que a normal, à direita, e caudas um pouco mais leves que a normal, à esquerda. Portanto, o uso de ondaletas deformadas parece apropriado.

A seguir, é ajustado um modelo de regressão não paramétrica usando as ondaletas adaptativas de Haar e limiar suave específico para dependência $\alpha$-mixing dos dados.

Na Figura 4.5 (a), observa-se a estimativa obtida com a ondaleta adaptativa de Haar. Os dados extremos parecem influenciar significativamente a estimativa. Para verificar este fato, 

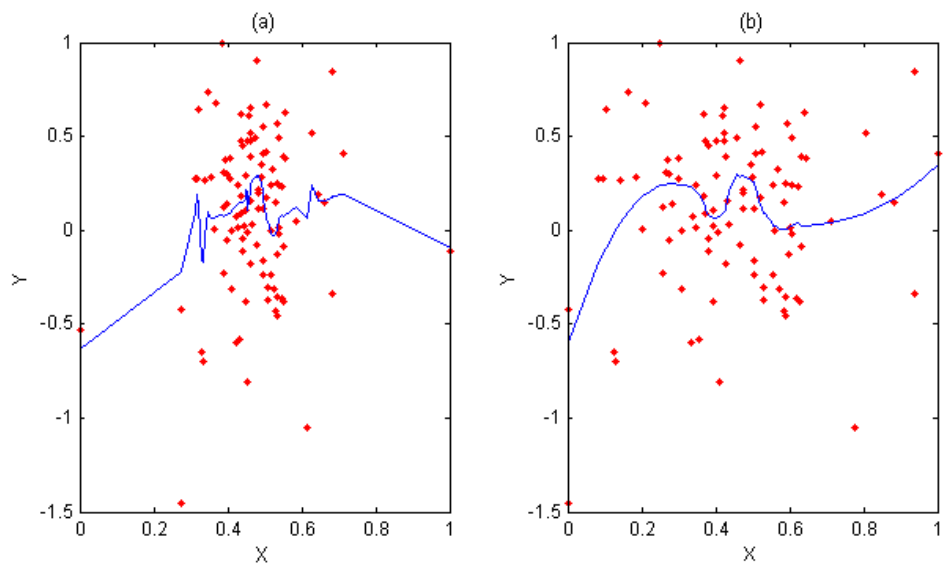

Figura 4.5: Gráfico de dispersão entre os retornos das ações ARCZ6 e retornos do Ibovespa:(a) a linha sólida representa a estimativa obtida com ondaletas adaptativas de Haar com todos os dados; (b) a linha sólida representa a estimativa obtida com ondaletas adaptativas de Haar tirando o máximo e o mínimo dos dados.

foram retirados o máximo e o mínimo do conjunto de dados e feitos os ajustes novamente, obtendo-se a curva da Figura 4.5 (b).
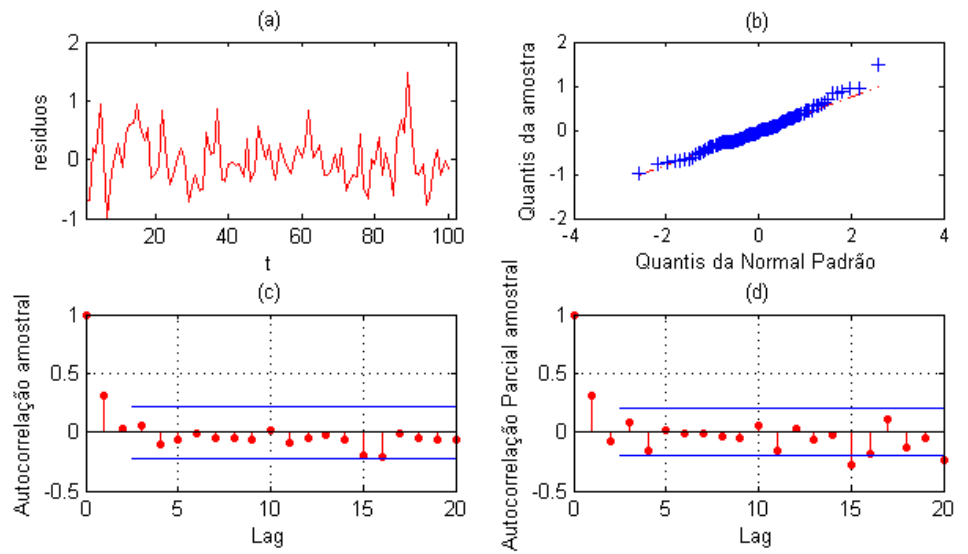

Figura 4.6: Gráficos dos resíduos obtidos com ondaletas adaptativas: (a) gráfico da série de resíduos, (b) gráfico de quantis, (c)função de autocorrelação, (d) função de autocorrelação parcial.

Os resíduos para o ajuste, excluindo-se os valores extremos, apresentam comportamento de ruído branco, como mostram as Figuras 4.6(c) e 4.6(d). A Figura 4.6 (b) apresenta o gráfico de quantis, o qual não indica grandes desvios da hipótese de normalidade. Portanto, conclui-se 
que é adequado o uso de ondaletas adaptativas de Haar para ajustar o modelo.

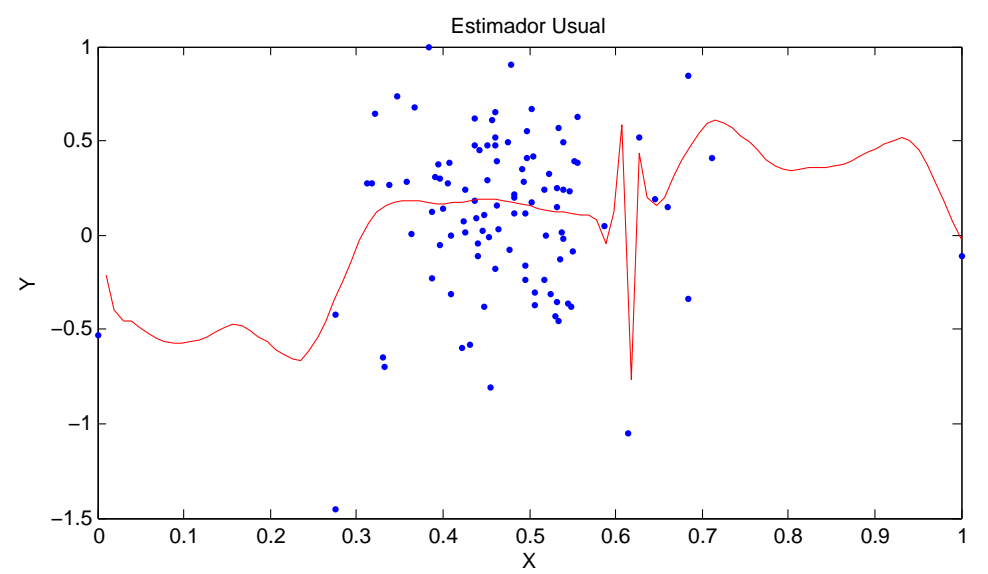

Figura 4.7: Gráfico de dispersão entre os retornos das ações ARCZ6 e retornos do IBOVESPA, a linha solida representa a estimativa obtida com ondaletas usuais.

Ao usar uma base de ondaleta que não leva em conta o desenho desigualmente espaçado dos dados e a estrutura de dependência, obtém-se a estimativa da Figura 4.7, quando se aplica ondaleta usuais com base Symmlet6. Observa-se que não se obtém um bom ajuste conforme as Figuras 4.8 (c) e $4.8(\mathrm{~d})$.

\subsection{Aplicação 2, Modelo de Índice Único}

O modelo de regressão clássica tem a seguinte forma:

$$
y_{t}=X_{t} \beta+\epsilon_{t}, \quad t=1,2, \ldots, N,
$$

onde $X_{t}=\left\{x_{1}, \ldots, x_{p}\right\}$ é uma matriz de p-variáveis, $\beta=\left(\beta_{1}, \beta_{2}, \ldots, \beta_{p}\right)^{T}$ é o vetor de parâmetros desconhecidos, e os $\epsilon_{t}$ são variáveis aleatórias com media zero e variância condicionada em $X_{t}$ limitada. Em alguns casos, os dados não podem ser analisados por meio de regressão linear. Isso torna necessário o uso da regressão não linear, o que apresenta como problema a dimensionalidade dos dados. Aqui são considerados Modelos de Índice Único, os quais superam a desvantagem da dimensionalidade e são frequentemente usados em Econome- 

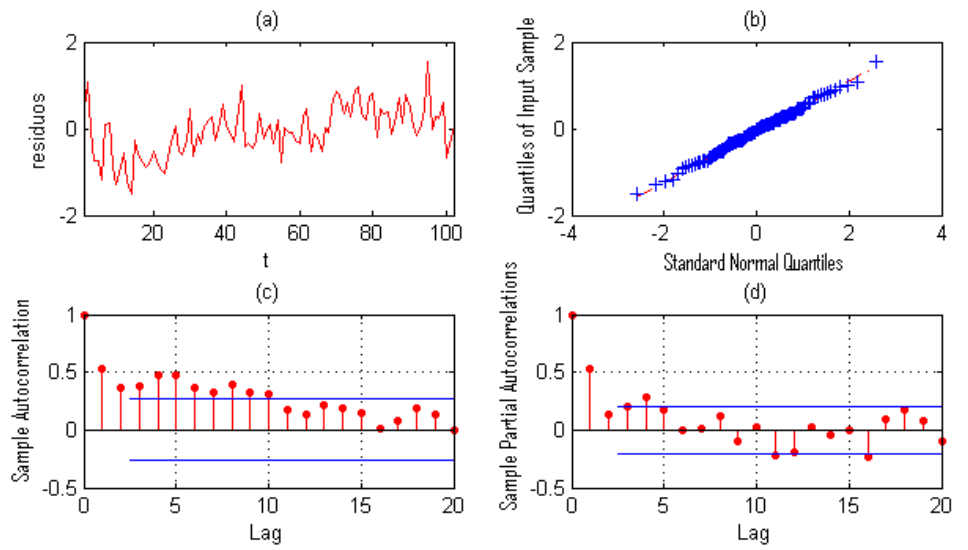

Figura 4.8: Gráficos dos resíduos obtido aplicando ondaletas usuais com base symlet6, (a)Gráfico dos resíduos, (b) quantil, (c)função de autocorrelação dos resíduos, (d) função de autocorrelação parcial dos resíduos.

tria.

Os Modelos de Índice Único, Stoker (1986), são um tipo especial de regressão não linear que explora a relação de uma variável resposta $y_{t}$ e de uma matriz de p-variáveis $X$ da forma:

$$
y_{t}=f\left(X^{\prime} \beta\right)+\epsilon_{t}, \quad t=1,2, \ldots, N,
$$

onde $E\left[y_{t} \mid X_{t}\right]=f\left(X^{\prime} \beta\right), \operatorname{Var}\left(y_{t} \mid X_{t}\right)<\infty, \beta$ é um vetor $p \times 1$ de parâmetros desconhecidos, $f($.) é uma função desconhecida e $\|\beta\|=1$, uma condição para tornar o modelo identificável. O modelo (4.4) é equivalente ao tradicional modelo de regressão não paramétrica onde o escalar $X^{\prime} \beta$ é chamado Índice. O objetivo é estimar $\beta$ e $f($.$) .$

\subsubsection{Representação em Ondaletas do Modelo de Índice Único}

Seja $f$ a função do modelo (4.4) e seja $Z=X^{\prime} \beta$. Um estimador não paramétrico de ondaletas para $f$ é construído como uma projeção ortogonal da função em um subconjunto de $L_{2}(\mathbb{R})$ como

$$
\hat{f}(Z)=\sum_{k \in \mathbb{Z}} \hat{\alpha}_{j_{0} k} \phi_{j_{0} k}(Z)+\sum_{j=0}^{J_{1}} \sum_{k=0}^{2^{j}-1} \hat{\beta}_{j k} \eta_{H}\left(\hat{\beta}_{j, k}, t_{j k}(Z)\right) \psi_{j k},
$$


onde $j_{0} \leq m-1$ e $\eta_{H}\left(\hat{\beta}, t_{j k}\right)$ é o limiar para suavizar o estimador. O objetivo de estimar $\mathrm{f}$ pelo método de expansão em ondaletas é encontrar uma $\hat{f}$ que minimize o risco, como discutido no Capítulo 1, para alguma classe de funções $f$.

Como $\hat{f}$ depende do valor de $\hat{\beta}$ estimado, vamos precisar de um método iterativo para estimar $\beta$ e $f$, satisfazendo as condições de $\|\beta\|=1$ e $\beta_{1}>0$ e, com isso, tornar o modelo identificável. A estimação do Modelo de Índice Único consta de dois passos: primeiro, estima-se o vetor de parâmetros $\beta$ e, assim, obtém-se o índice $Z=X^{\prime} \hat{\beta}$. A seguir, é estimada a $f$ por métodos não paramétricos.

Park et al. (2005) usa a metodologia de ondaletas e métodos Bayesianos para este fim. Neste trabalho, é proposto o seguinte processo iterativo para obter as estimativas do Modelo de Índice Único.

1) É dado um valor inicial $\beta_{0}$, que é um vetor de três componentes, onde $0<\beta_{01}<1$, $0<\beta_{02}<1$ e $-1<\beta_{03}<0$. Na prática, cada valor de $\beta_{01}$ e $\beta_{02}$ é gerado com uma uniforme $(0,1)$ e $\beta_{03}$ é gerado com uma uniforme(-1,0). Também é considerada, como valor inicial, $\hat{f}_{0}=y_{t}$;

2) um valor $\beta_{1}$ é estimado pelo método de mínimos quadrados, com a condição de que $\|\beta\|=1$.

Na prática, este passo é implementado no MATLAB usando a função fmincon, tomando como valor inicial o $\beta_{0}$ dado no passo anterior. A fmincon permite encontrar mínimos locais de funções em várias variáveis com restrições não lineares. É especificado o método de mínimos quadrados para otimizar a função;

3) é calculado o valor $X^{\prime} \hat{\beta}_{1}$ e aplicado o método de ondaletas para estimar $\hat{f}_{1}\left(X^{\prime} \hat{\beta}_{1}\right)$, tal que (1.4) seja minimizada;

4) se $\left\|\hat{f}_{0}-\hat{f}_{1}\right\|>\epsilon$, voltamos ao passo 2 e agora o valor inicial é o valor de $\hat{\beta}_{1}$ obtido no passo anterior. Assim um novo valor de $\hat{\beta}_{2}$ é estimado. E, conforme feito no passo 3, é obtida $\hat{f}_{2}$. Novamente, são comparados $\hat{f}_{2}$ e $\hat{f}_{1}$. Se $\left\|\hat{f}_{1}-\hat{f}_{2}\right\|>\epsilon$, o processo é repetido. Com isso, é obtida uma sequência de estimativas $\hat{f}_{1}, \ldots, \hat{f}_{j-1}, \hat{f}_{j}$. O processo é finalizado 
quando $\left\|\hat{f_{j-1}}-\hat{f}_{j}\right\|<\epsilon$, onde $\epsilon$ é um valor pequeno.

Como a função fmincon só encontra mínimos locais, usamos uma serie de $\mathrm{N}$ valores iniciais que possam garantir a cobertura da região $0<\beta_{1}<0,0<\beta_{2}<1$ e $-1<\beta_{3}<0$. Então, são obti$\operatorname{dos} \hat{\beta}_{1}, \ldots, \hat{\beta}_{N}$, estimadores de $\beta$, e tomamos como estimador final a média dos $\mathrm{N}$ estimadores. Assim, obtemos um valor de $X^{\prime} \hat{\beta}$ e estimamos a função f pela expansão de ondaletas deformadas ou adaptativas.

\subsubsection{Aplicação a Dados de Poluição}

Nesta aplicação, é considerado um estudo ambiental onde a concentração de ozônio, $y$, depende de três variáveis meteorológicas, $X_{1}$ : temperatura, $X_{2}$ : radiação e $X_{3}$ : velocidade do vento. Os dados são medições de 111 dias, de maio a setembro de 1973, na região metropolitana da cidade de New York. O objetivo dos dados é conseguir explicar o comportamento da poluição através das variáveis temperatura, radiação e velocidade do vento. Para isto, é usado o Modelo de Índice Único.

Na Figura 4.9 são apresentados os gráficos das quatro variáveis. Observa-se que as variáveis ozônio e Temperatura apresentam variância menor no início e ao final do período observado, enquanto as variáveis radiação e velocidade do vento apresentam um comportamento estacionário. Para deixar todas a séries com um comportamento estacionário, foram feitas as seguintes transformações: para a variável temperatura foi tomado o logaritmo e depois feita uma diferença; para a variável ozônio foi aplicado o logaritmo. Desta forma, todas as variáveis (originais e transformadas) têm comportamento estacionário.

Com a metodologia do Modelo Índice Único, primeiro será aplicada a ondaleta deformada, usando várias bases e valores de k, para saber qual base e valor de k são mais adequado para aplicar a transformada. Para isso, foram feitas estimativas de $\beta$ pelo procedimento sqlin do MATLAB (recurso que possibilita encontrar as estimativas do vetor $\beta$ ), com as seguintes restrições: $0<\beta_{1}<1,0<\beta_{2}<1$ e $-1<\beta_{3}<0$. Com o valor de $\hat{\beta}$ encontrado, é calculado $X^{\prime} \hat{\beta}$ e logo aplicada a transformada de ondaletas deformadas com as bases Symmlet6, Symmlet7, Symmlet8, Symmlet9, Symmlet10 e coiflet2, coiflet3, coiflet4, coiflet5. Observamos o valor do RMSE e o comportamento dos resíduos, para valores de $k=0,6 ; 0,7 ; 0,8 ; 0,9 ; 1 ; 1,1 ; 1,2 ; 1,3$ 
(a)

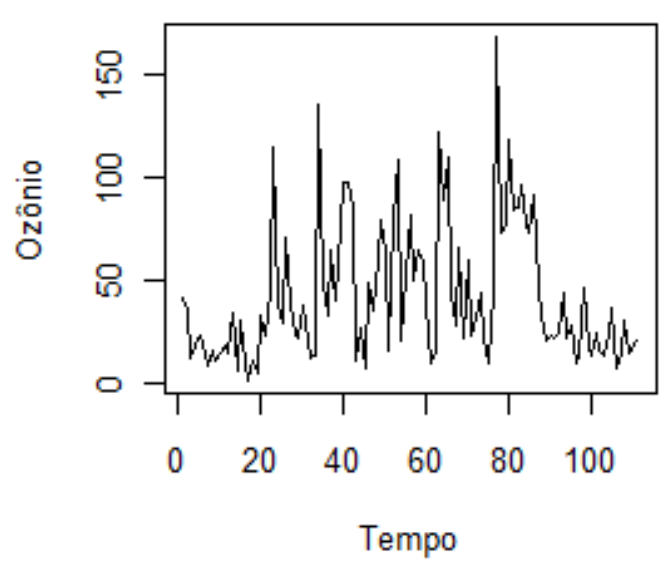

(c)

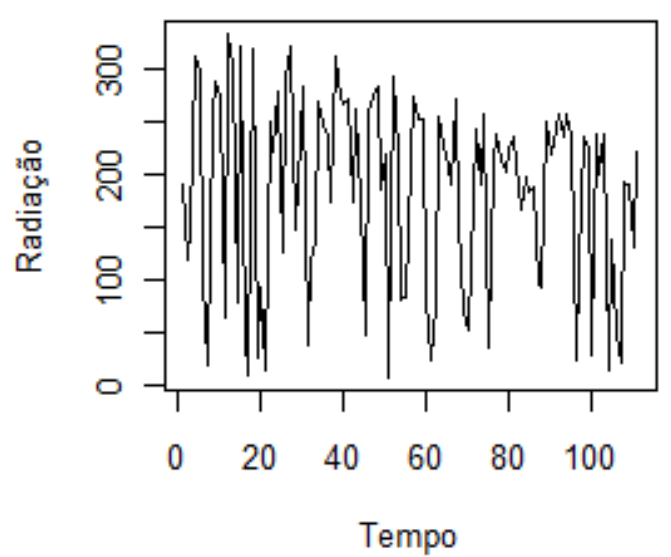

(b)

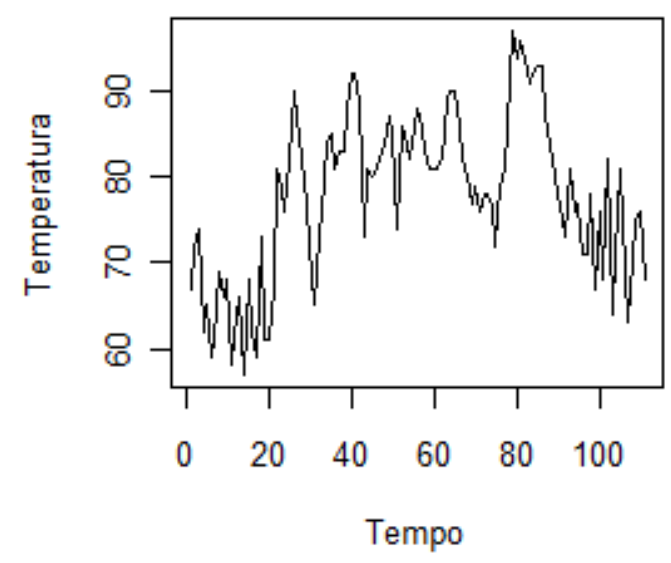

(d)

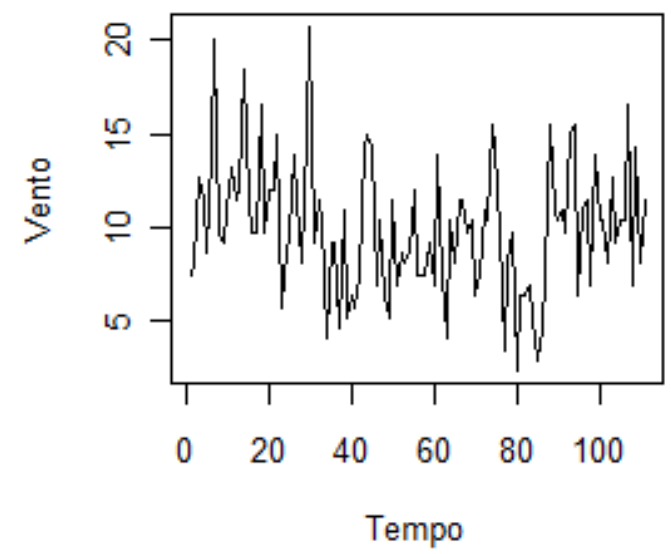

Figura 4.9: Funções: (a) gráfico da serie ozônio, (b)gráfico da série temperatura, (c) gráfico da série radiação, (d) gráfico da série velocidade do vento.

e 1,4. As bases que tiveram melhor comportamento foram sym7 e coif5 para valores de $\mathrm{k}=0,9$, 1 e 1,1. A curva ficou mais suave com valor de $\mathrm{k}=0,9$ e para as bases Symmlet7 e Coiflet5, de modo que todo o processo iterativo de estimação será aplicado para $k=0,9$ e base Symmlet7. De forma geral, os mesmos apresentaram melhor ajuste.

Na Figura 4.10 (a) são apresentados os gráficos dos boxplot para cada componente do vetor de parâmetros. Observa-se que os estimadores não variam muito e, tomando a média de cada componente, obtém-se o estimador $\hat{\beta}=(0,4891,0,1657,-0,8077)$. A Figura 4.10 (b) apresenta o gráfico de dispersão de $X^{\prime} \hat{\beta}$, que evidencia um delineamento desigualmente espaçado e, para valores maiores de X, se tem maior concentração de valores de Y. A Figura 4.10 (c) apresenta 
(a)

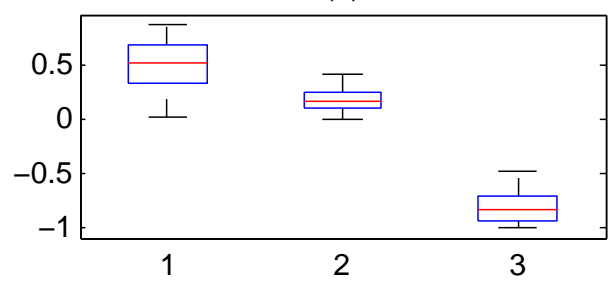

(c)

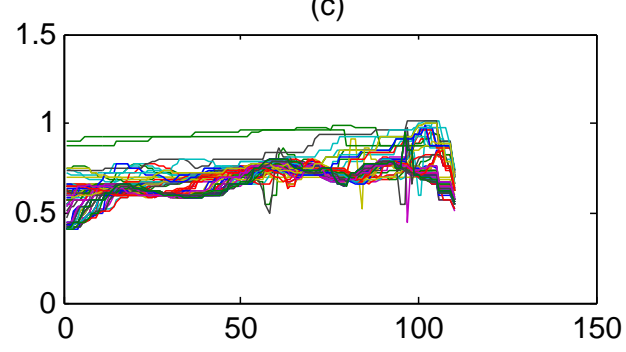

(b)

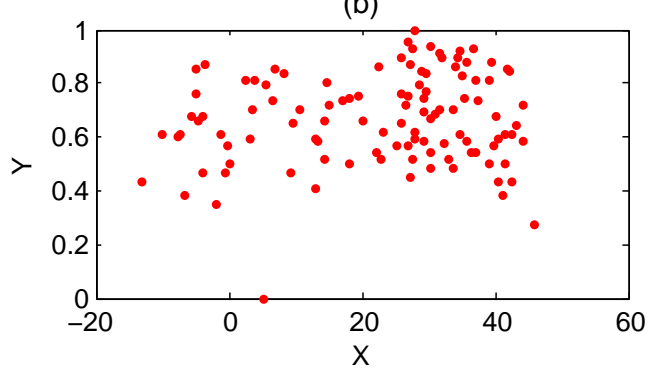

(d)

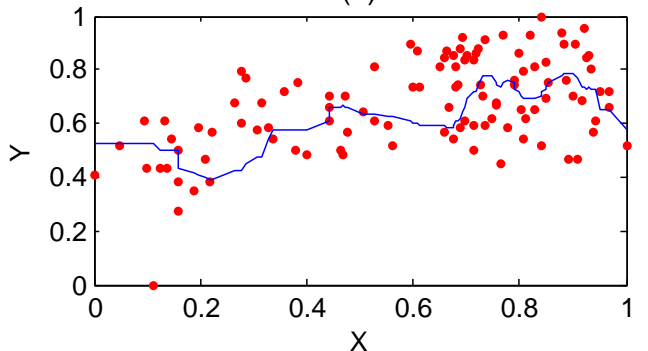

Figura 4.10: (a) boxplot das estimativas de $\beta_{1}, \beta_{2}$ e $\beta_{3}$, respectivamente; (b) gráfico da série $X^{\prime} \hat{\beta}$ contra a série ozônio; (c) gráfico das curvas ajustadas para cada estimativa obtida do valor inicial dado; (d) apresenta a $\hat{f}$ estimada para o valor de $\hat{\beta}$.

as diferentes $\hat{f}$ ajustadas pelas ondaletas deformadas, o que indica um comportamento similar em todas as estimativas da $f$. A Figura 4.10 (d) mostra a $\hat{f}$ estimada para o valor de $\hat{\beta}=(0,4891,0,1657,-0,8077)$, o que evidencia uma tendência não linear para a relação entre o índice e a variável ozônio.

A Figura 4.11 (a) apresenta a função da autocorrelação amostral dos resíduos depois de fazer o ajuste de ondaletas, que evidencia um comportamento de ruído branco. A Figura 4.11 (b) apresenta o gráfico de $Q \times Q$, que mostra que a hipótese de resíduos com distribuição Normal é razoável.

O valor do RMSE para a estimativa da ondaleta é 0,1599. Portanto, pode-se concluir que é adequado usar a metodologia de ondaletas deformadas para ajustar um modelo de regressão não linear aos dados.

Aplicando a metodologia de ondaletas adaptativas de Haar aos dados, com o Modelo de Índice Único, como explicado anteriormente, foi usado um método de estimação iterativo para encontrar o valor de $\hat{\beta}$. 

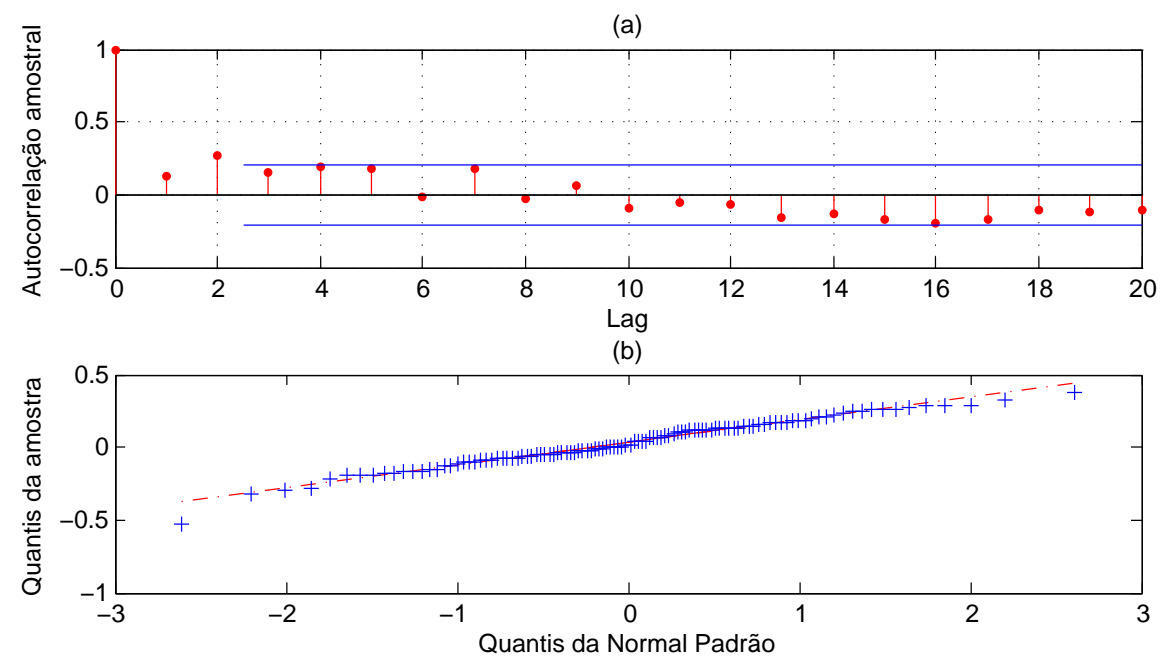

Figura 4.11: (a) Gráfico da função de autocorrelação amostral dos resíduos, (b) Gráfico Quantil-Quantil dos resíduos.

(a)

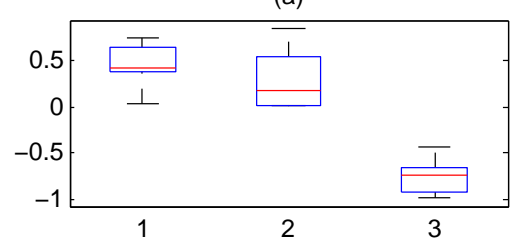

(c)

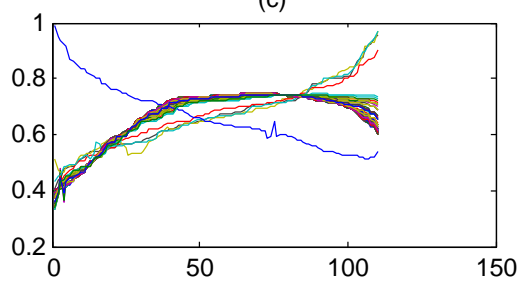

(b)

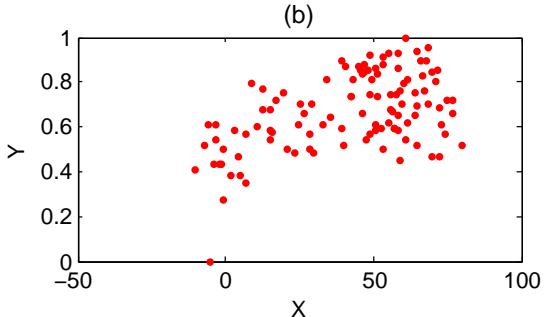

(d)

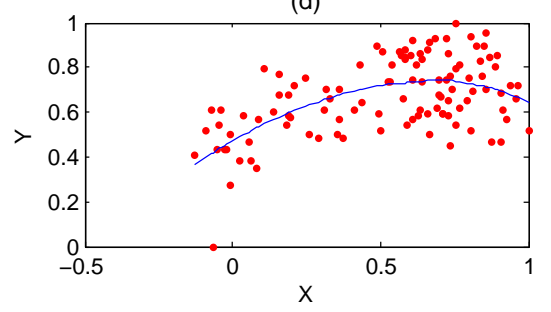

Figura 4.12: (a)boxplot das estimativas de $\beta_{1}, \beta_{2}$ e $\beta_{3}$, respectivamente; (b)gráfico da série $X \hat{\beta}$ contra a série ozônio; (c)gráfico das curvas ajustadas para cada estimativa obtida do valor inicial dado; (d)apresenta a $\hat{f}$ estimada para o valor de $\hat{\beta}$.

Na Figura 4.12 (a) são apresentados os boxplot dos estimadores $\beta_{1}, \beta_{2}$ e $\beta_{3}$, respectivamente. O valor do vetor estimado é $\hat{\beta}=(0,4630,0,2660,-0,7637)$. Na figura 4.12 (c), temos as curvas ajustadas para cada um dos 100 valores de $\hat{\beta}$ encontrados pelo método iterativo. Em geral, observa-se um comportamento similar nas funções estimadas, mais depois da observação 85 o comportamento diverge um pouco. Na Figura 4.12 (d) a linha contínua representa a estimativa 
obtida pela ondaleta adaptativa de Haar, mostrando uma tendência crescente não linear nos dados, para valores do Indice $X$ menores que 0,6 , logo apresenta um comportamento decrescente.
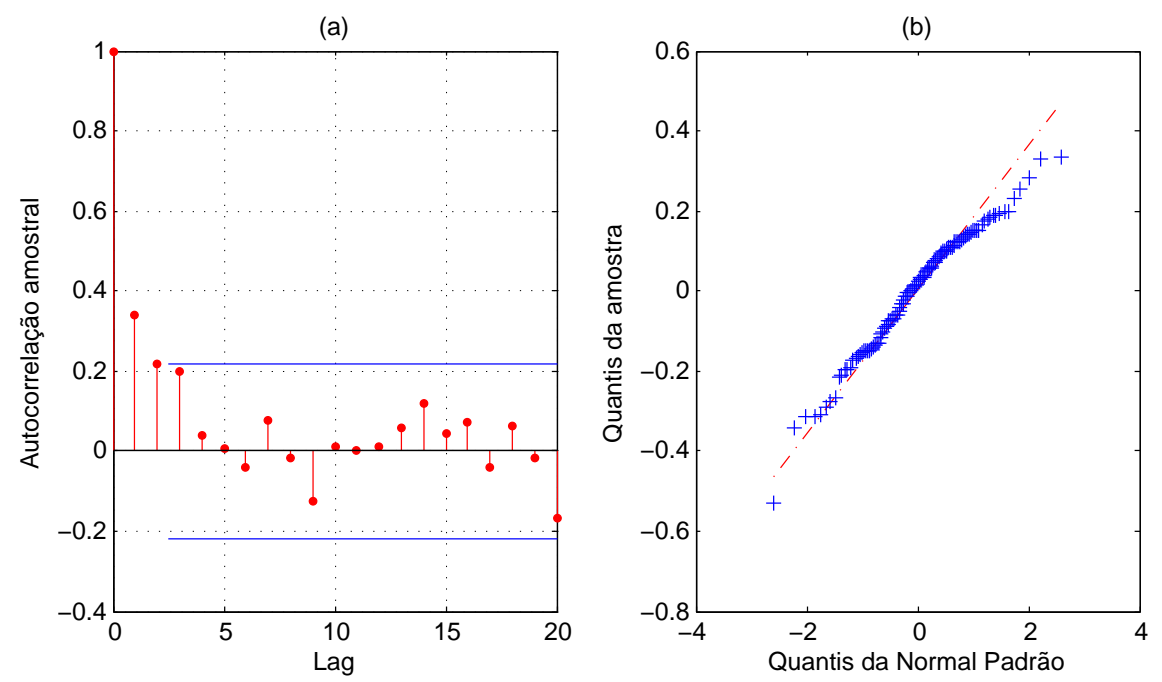

Figura 4.13: (a) Gráfico da função de autocorrelação amostral dos resíduos; (b) Gráfico Quantil-Quantil dos resíduos.

Na figura 4.13 (a), apresenta-se a fac dos resíduos, mostrando uma correlação serial desprezível, exceto no lag 1. Quanto à suposição de normalidade o gráfico $Q \times Q$ não indica grande desvio da suposição de normalidade. O valor de RMSE para a estimativa de ondaleta é 0,1620 . Portanto parece adequado usar a metodologia de ondaleta adaptativa de Haar para ajustar os dados a um modelo não linear. 


\section{Capítulo 5}

\section{Conclusões}

Nesta tese, o interesse foi obter taxas de convergência, em regressão não paramétrica, quando a variável explicativa $X_{t}$ é um processo estacionário $\alpha$-mixing, com o delineamento desigualmente espaçado, em particular, com os procedimentos de ondaletas deformadas e ondaletas adaptativas de Haar. Foram apresentadas condições sobre o processo $X_{t}$ e sua estrutura de dependência, assim como do domínio da função f a ser estimada. Também foram apresentadas as adaptações necessárias aos procedimentos para obter as taxas de convergência do risco para a norma $L_{p}$, no caso de ondaletas deformadas. Em relação às ondaletas adaptativas de Haar, obtém-se as taxas de convergência do risco do estimador proposto.

Mediante estudos de simulação, foram avaliados os comportamentos dos métodos propostos quando aplicados a amostras finitas. Em geral, os métodos apresentam um bom desempenho quanto ao ajuste das curvas.

Para ondaletas deformadas, quando a variável explicativa $X_{t}$ tem uma estrutura de dependência $\alpha$-mixing, com limiar universal duro, o procedimento proposto apresenta boas taxas de convergência. Neste caso é exigido normalidade dos erros, mas a classe de funções é ampla.

O procedimento usando ondaletas adaptativas de Haar, com limiar universal modificado, apresenta boas taxas de convergência e produz estimadores suaves. O limiar modificado permite obter estimativas mais adequadas para os dados dependentes, já que os resíduos apresentam comportamento mais próximo ao ruído branco, quando comparado ao uso do limiar universal usual.

Quando os procedimentos usando ondaletas deformadas e ondaletas adaptativas de Haar foram aplicados a dados reais, em um modelo de regressão, obtivemos estimativas razoáveis e mais adequadas do que as obtidas com ondaletas usuais. Quando as metodologias foram aplicadas 
a modelos um pouco mais complexos, como o Modelo de Índice Único, as estimativas obtidas também foram boas.

Entre as possibilidades de pesquisas futuras,podemos destacar:

- comparações entre os métodos;

- estudo de outras funções limiares;

- exploração de outras relações de dependência;

- estudo mais detalhado dessas metodologias de ondaleta para o Modelo de Índice Único;

- possibilidade de estender alguma dessas metodologias para processos de co-integração. 


\section{Apêndice A}

\section{Gráficos das simulações}

\section{A.0.3 Graficos das Simulações com Ondaletas Deformadas}

(a)

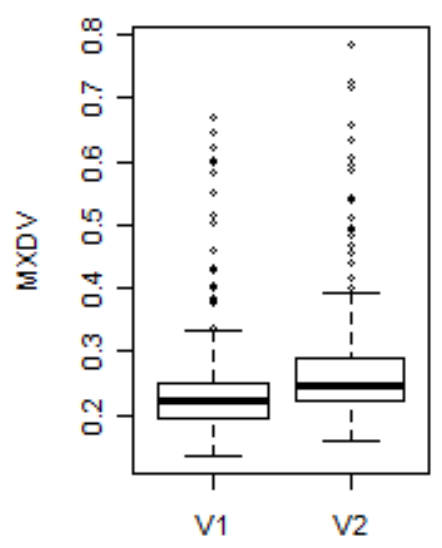

(d)

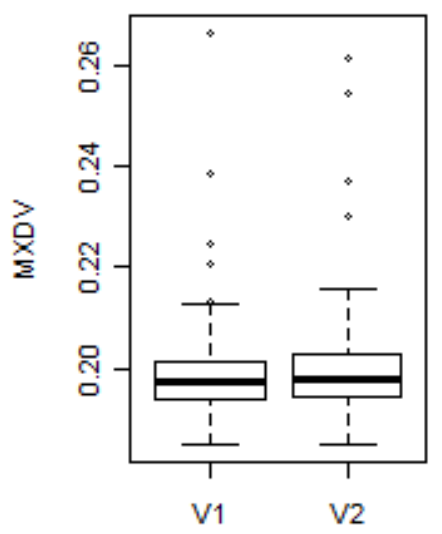

(b)

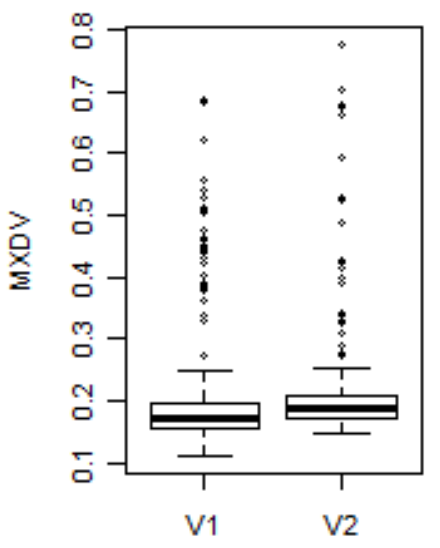

(e)

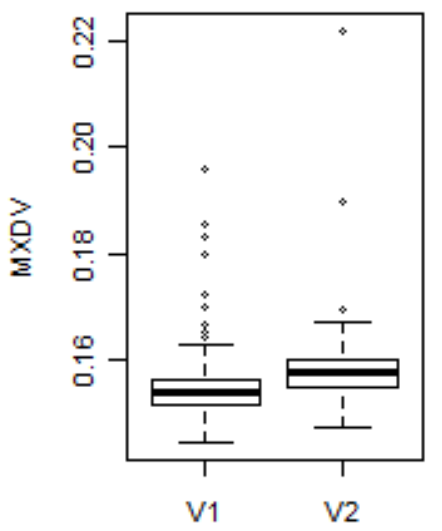

(c)

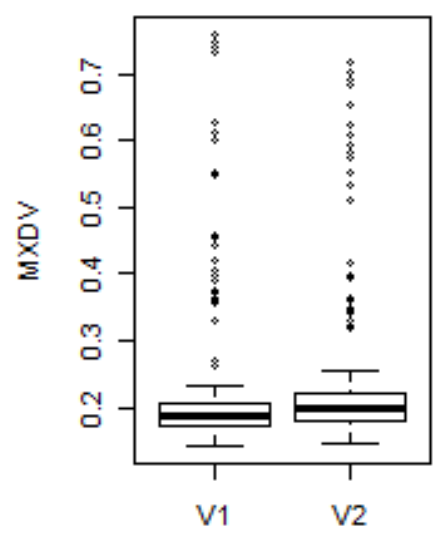

(f)

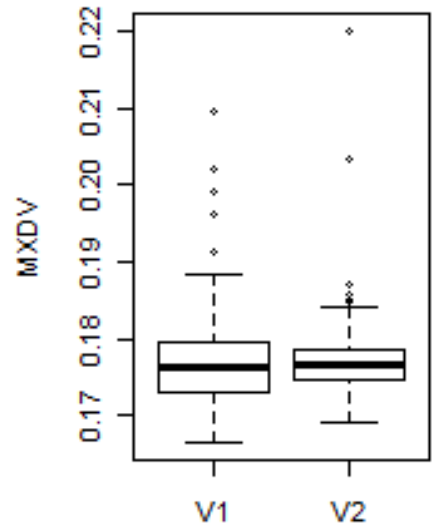

Figura A.1: Boxplot de MXDV para $f(x)=$ seno, $g(x)=$ constante, $(a),(b),(c)$ com $r s n=1$ e $N=$ 512,1024 e 2048, respectivamente, para $(d),(e),(f) r s n=7$ e $N=512,1024$ e 2048, respectivamente; V1 representa a base symmlet 8 e V2 representa a base coiflet 3 . 
(a)

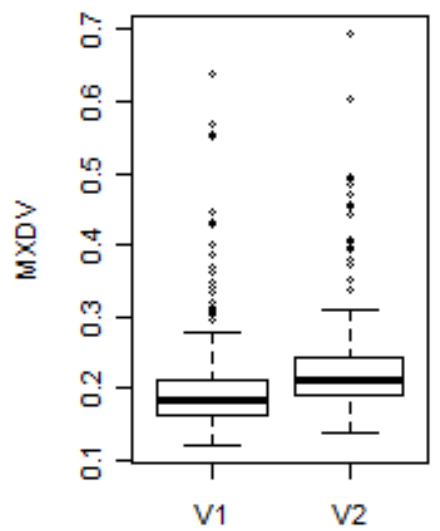

(d)

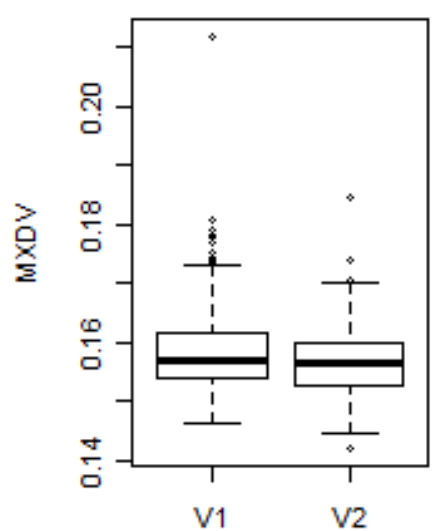

(b)

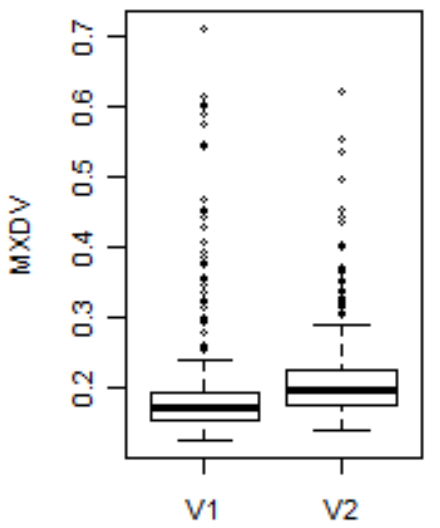

(e)

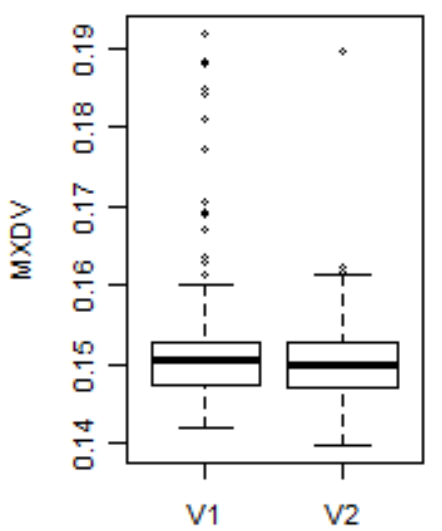

(c)

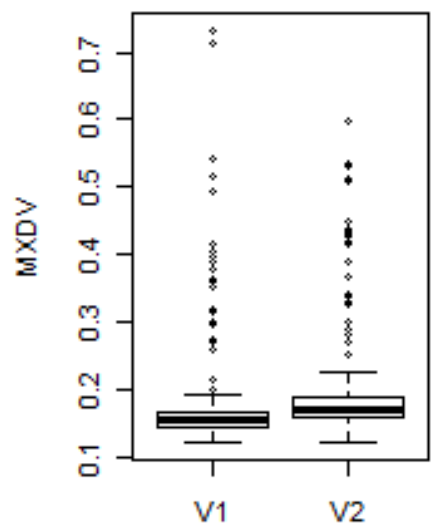

(f)

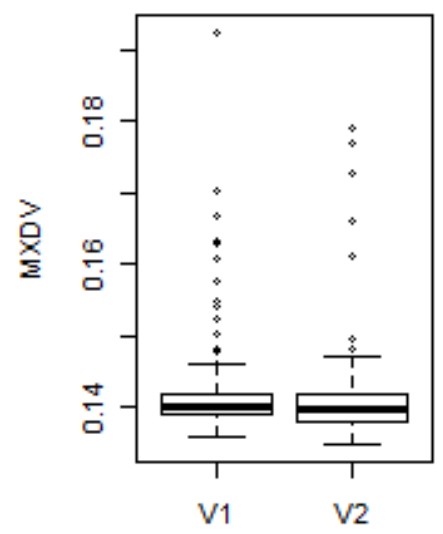

Figura A.2: Boxplot de MXDV para $f(x)=$ seno, $g(x)=$ seno, $(a),(b),(c)$ com $r s n=1$ e $N=512,1024$ e 2048, respectivamente, para $(d),(e),(f) r s n=7$ e $N=512,1024$ e 2048, respectivamente; V1 representa a base symmlet 8 e V2 representa a base coiflet 3 . 
(a)

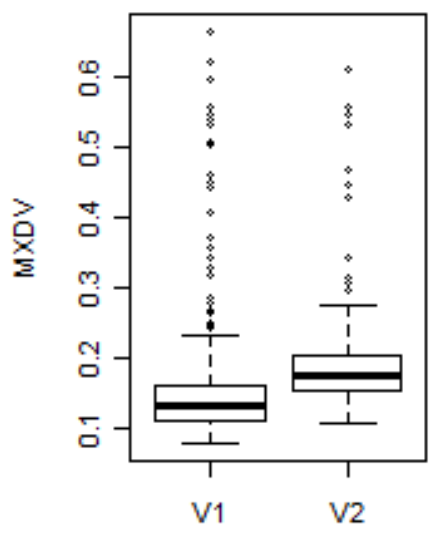

(d)

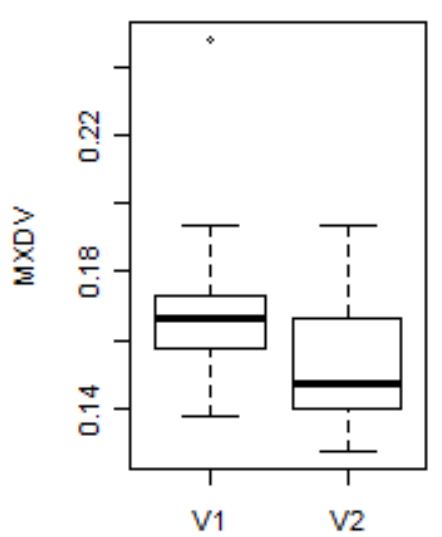

(b)

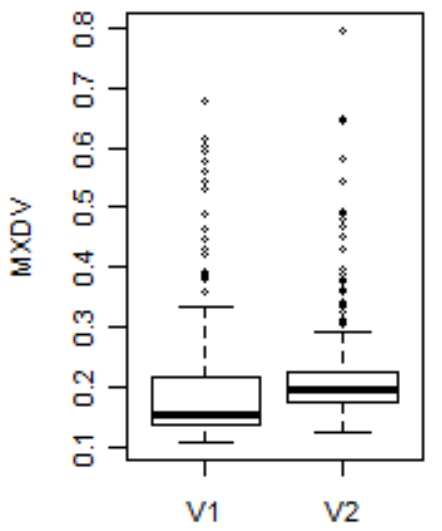

(e)

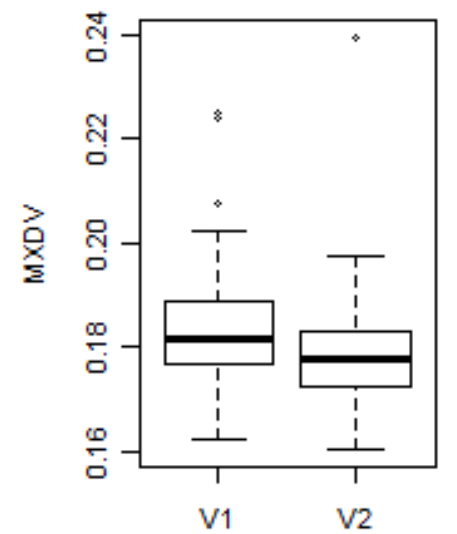

(c)

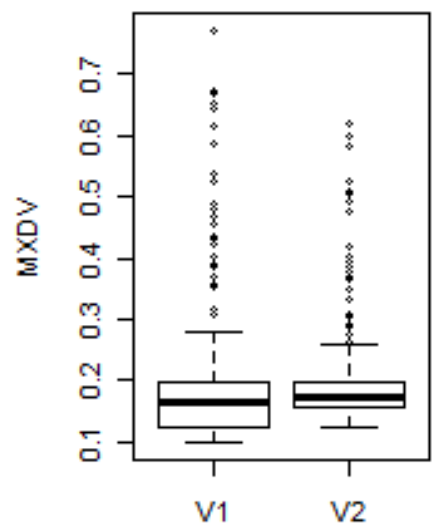

(f)

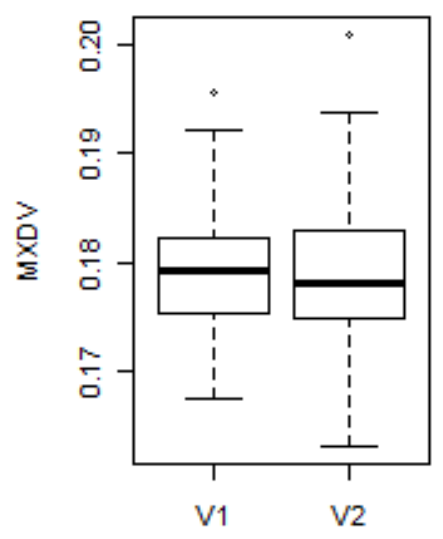

Figura A.3: Boxplot de MXDV para $f(x)=$ Heavisine, $g(x)=$ constante e para $(a),(b),(c) r s n=1$ e $N=512,1024$ e 2048, respectivamente, para $(d),(e),(f) r s n=7$ e $N=512,1024$ e 2048, respectivamente; V1 representa a base symmlet 8 e V2 representa a base coiflet 3 . 
(a)

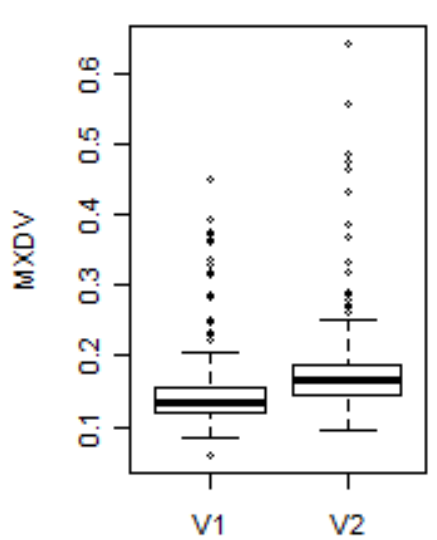

(d)

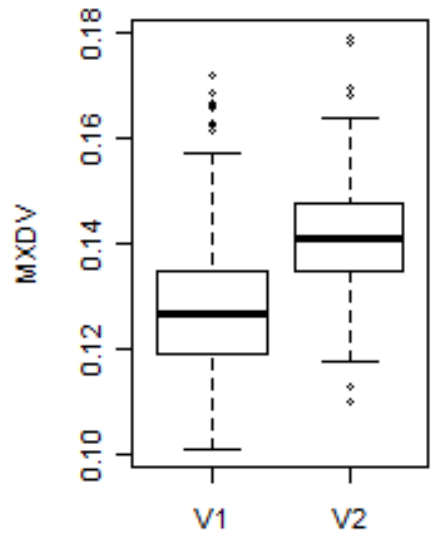

(b)

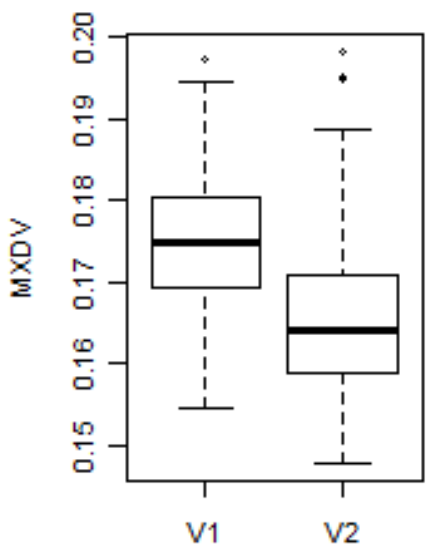

(e)

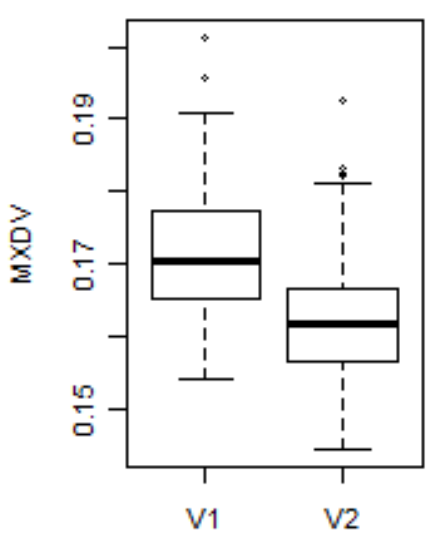

(c)

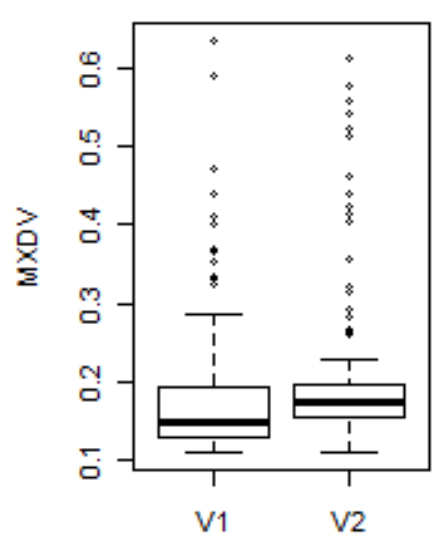

(f)

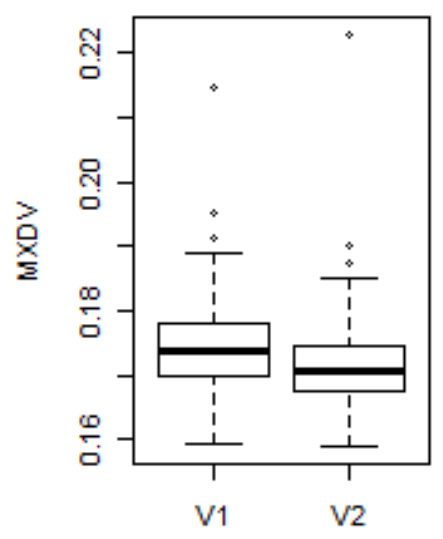

Figura A.4: Boxplot de MXDV para $f(x)=$ Heavisine, $g(x)=$ seno e para $(a),(b),(c) r s n=1$ e $N=$ 512,1024 e 2048, respectivamente, para $(d),(e),(f) r s n=7$ e $N=512,1024$ e 2048 , respectivamente;V1 representa a base symmlet 8 e V2 representa a base coiflet 3 . 
(a)

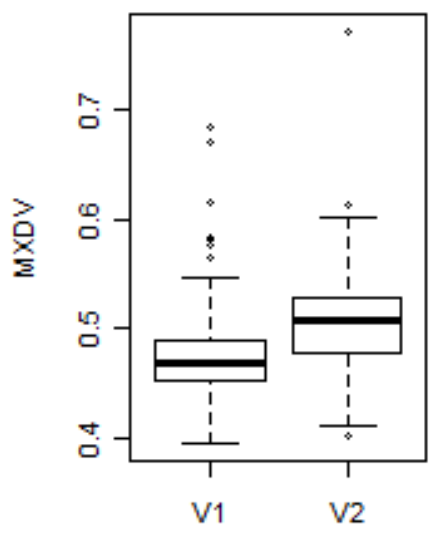

(d)

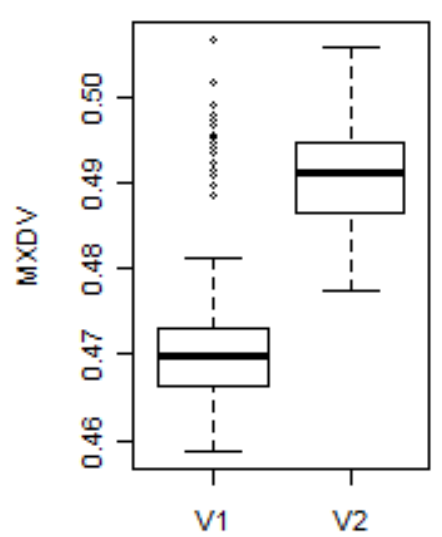

(b)

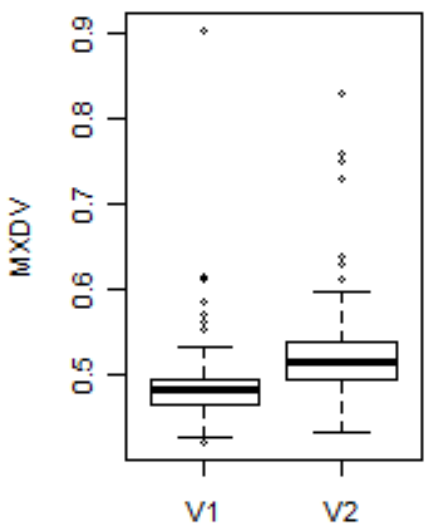

(e)

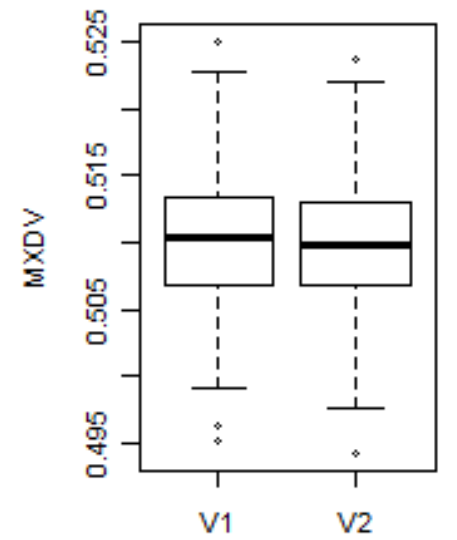

(c)

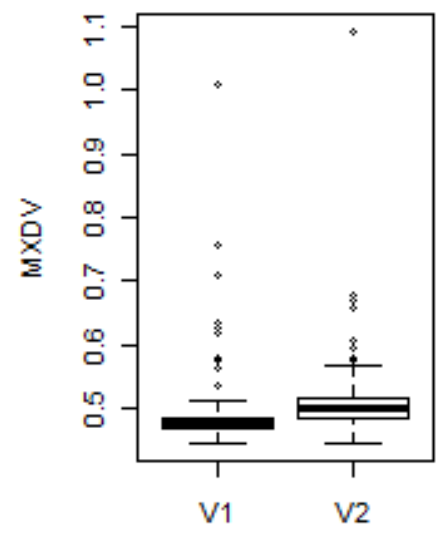

(f)

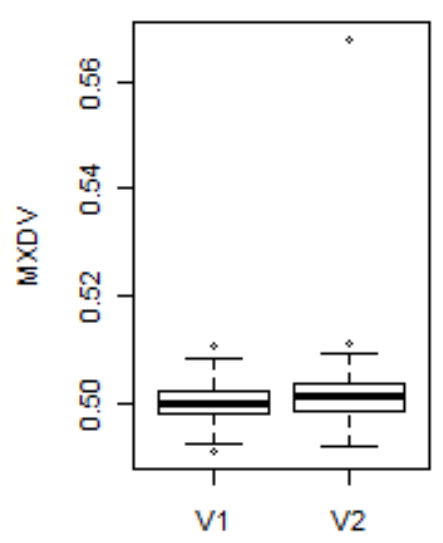

Figura A.5: Boxplot de MXDV para $f(x)=$ doppler, $g(x)=$ constante e para $(a),(b),(c) r s n=1$ e $N=$ 512,1024 e 2048, respectivamente, para $(d),(e),(f) r s n=7$ e $N=512,1024$ e 2048, respectivamente; V1 representa a base symmlet 8 e V2 representa a base coiflet 3 . 
(a)

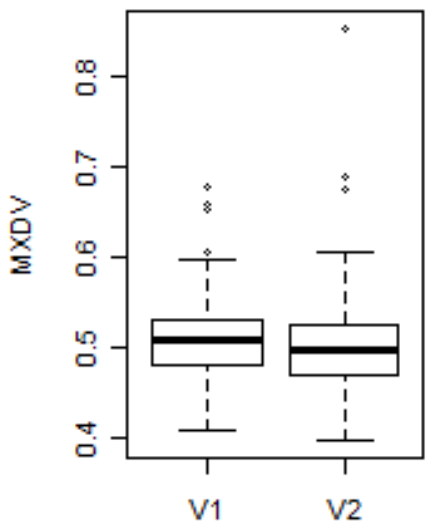

(d)

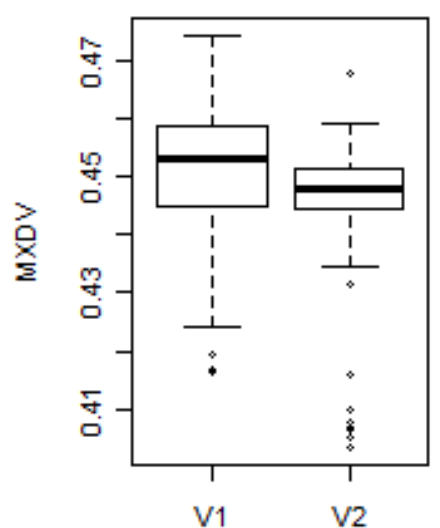

(b)

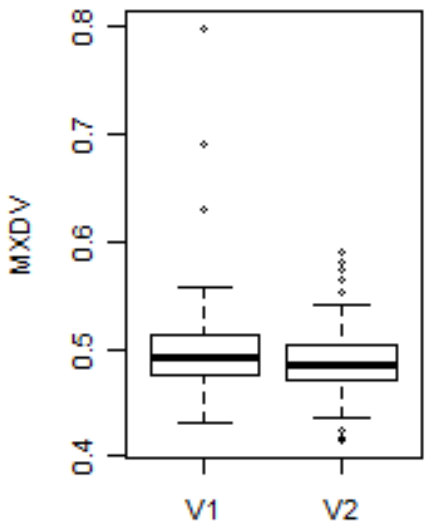

(e)

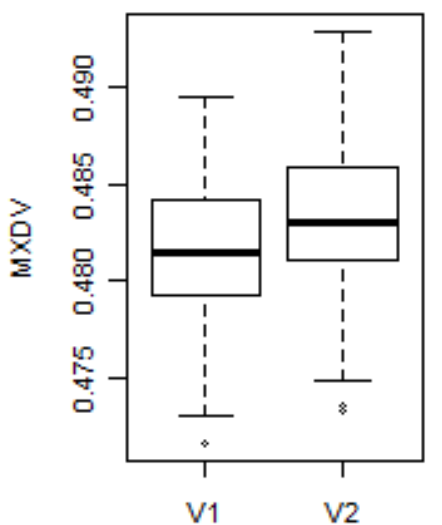

(c)

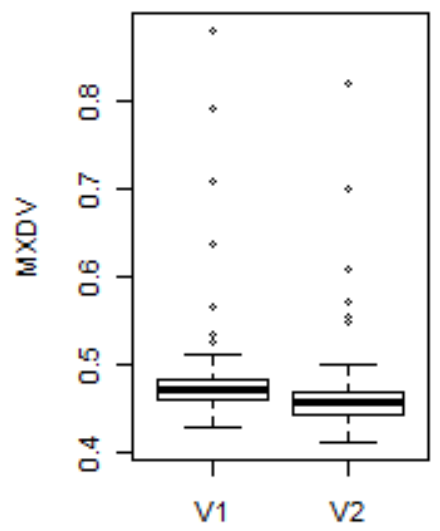

(f)

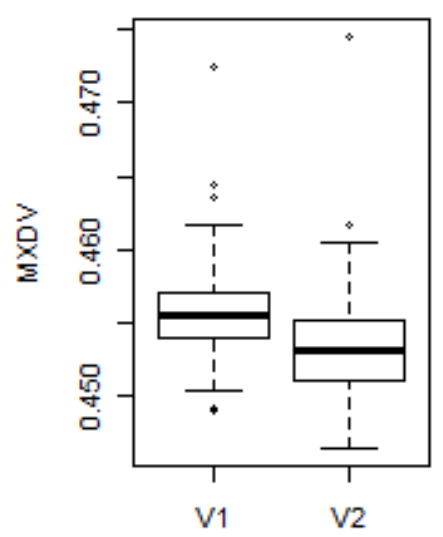

Figura A.6: Boxplot de MXDV para $f(x)=$ doppler, $g(x)=$ constante e para $(a),(b),(c) r s n=1$ e $N=$ 512,1024 e 2048, respectivamente, para $(d),(e),(f) r s n=7$ e $N=512,1024$ e 2048, respectivamente; V1 representa a base symmlet 8 e V2 representa a base coiflet 3 . 


\section{A.0.4 Graficos das Simulações com Ondaletas Adaptativas de Haar}

(a)

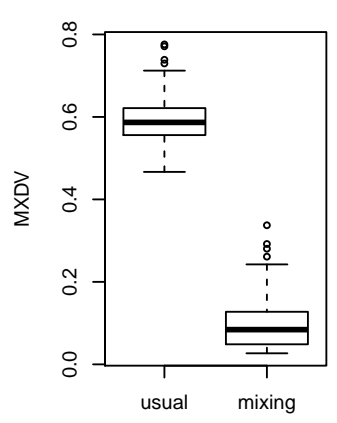

(d)

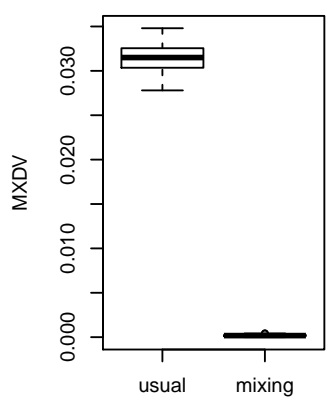

(b)

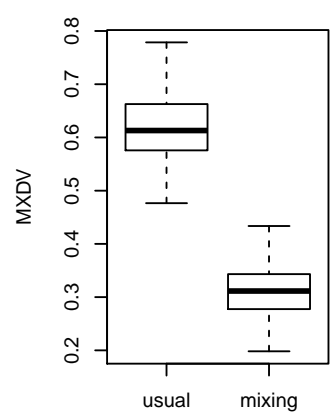

(e)

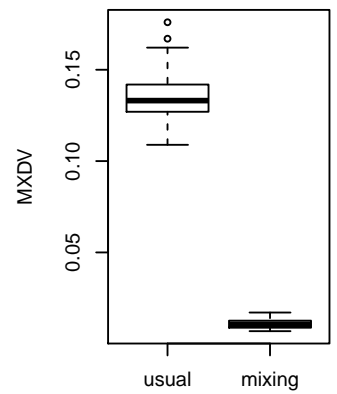

(c)

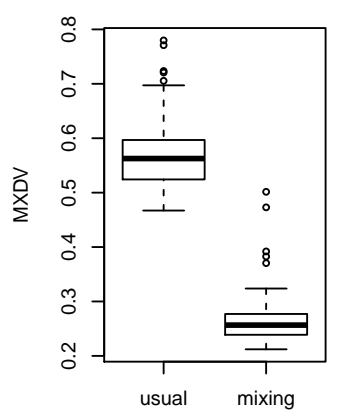

(f)

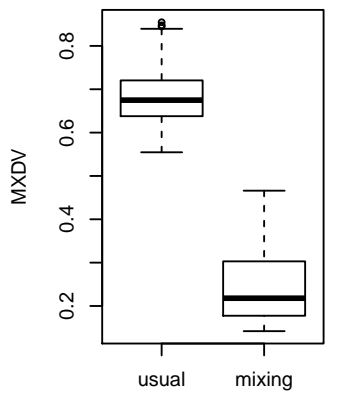

Figura A.7: Boxplot de MXDV para $\mathrm{n}=512$, snr $=1$ e $f(x)$ :(a) Seno, (b) Heavisine (c) Doppler, com snr $=7$ e $f(x)$ (a) Seno, (b) Heavisine (c) Doppler, aplicando limiar para dados $\alpha$-mixing, e limiar para dados independentes. 
(a)

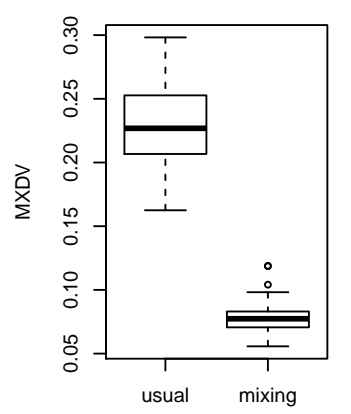

(d)

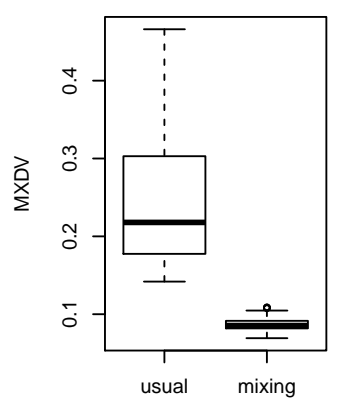

(b)

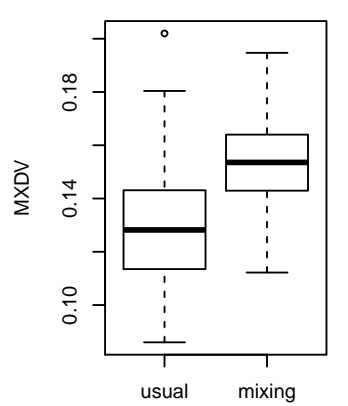

(e)

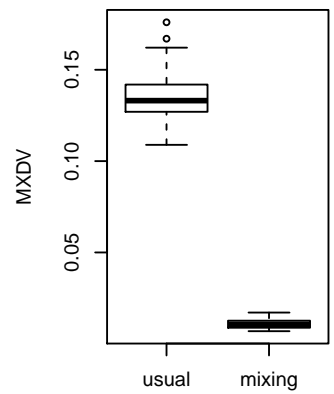

(c)

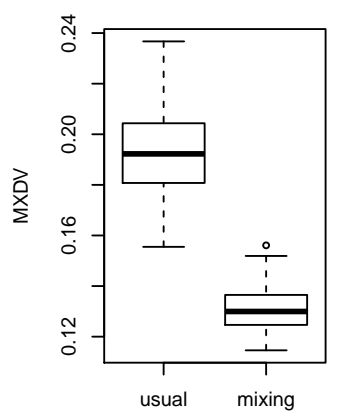

(f)

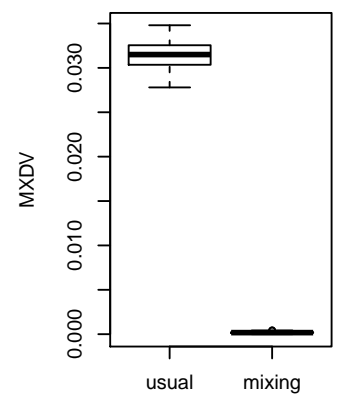

Figura A.8: Boxplot de MXDV para $\mathrm{n}=1024$, snr=1 e $f(x)$ :(a) Seno, (b) Heavisine (c) Doppler, com $\mathrm{snr}=7$ e $f(x)$ (a) Seno, (b) Heavisine (c) Doppler, aplicando limiar para dados $\alpha$-mixing, e limiar para dados independentes.

\section{A.0.5 Graficos das Aplicações}



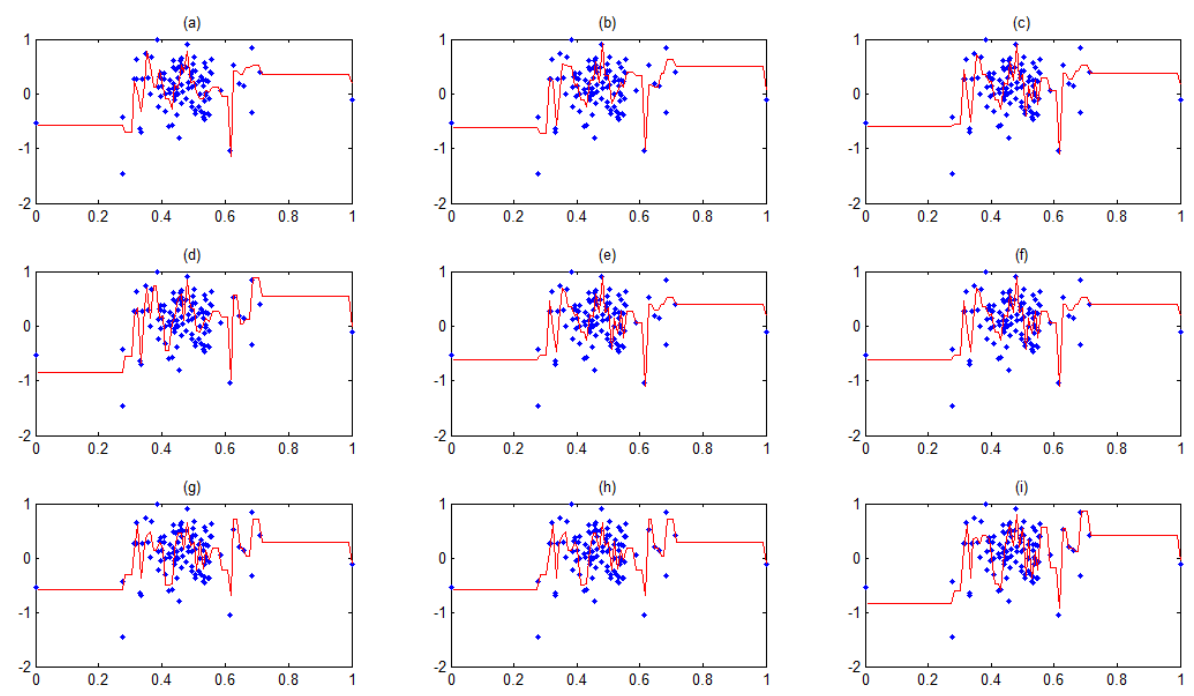

Figura A.9: Gráfico estimativas ondaleta deformada com $\mathcal{K}=0,5$ e base (a)Symmlet6, (b)Symmlet7, (c)Symmlet8, (d)Symmlet9, (e)Symmlet10, (f)Coiflet2, (g)Coiflet3, (h)Coiflet4, (i)Coiflet5.
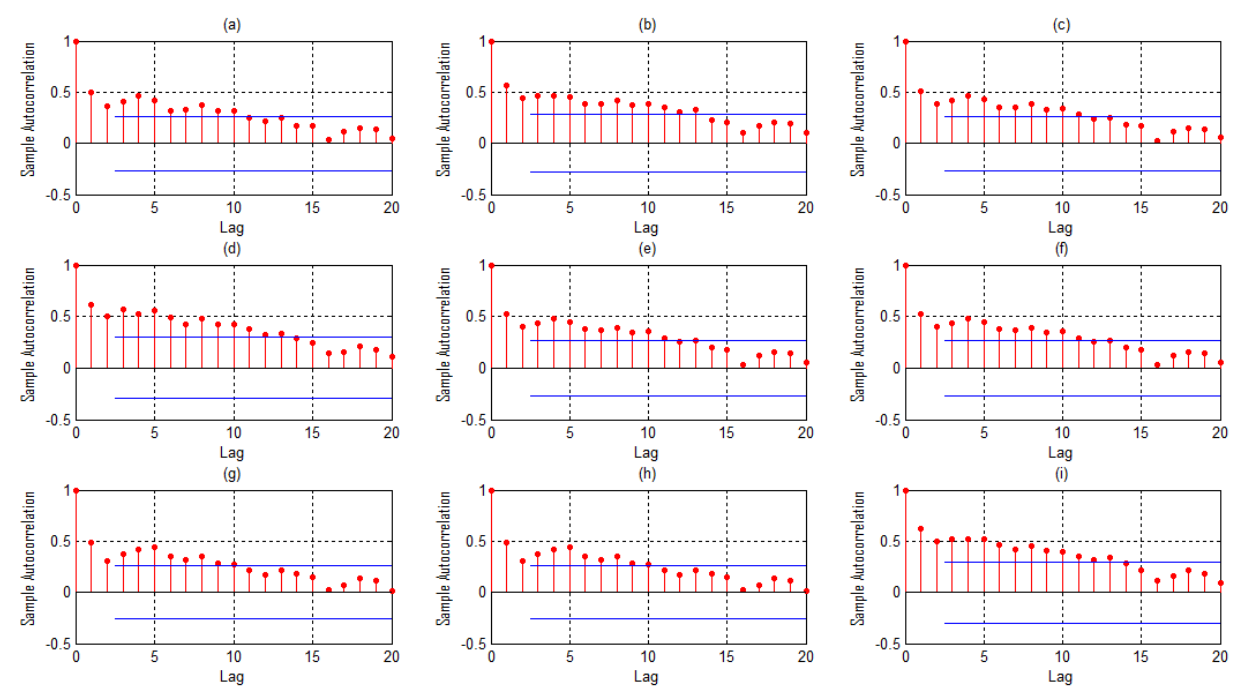

Figura A.10: Gráficos da função de autocorrelação dos resíduos das estimativas obtidas com ondaletas deformadas para $\mathcal{K}=0,5$ e base (a)Symmlet6, (b)Symmlet7, (c)Symmlet8, (d)Symmlet9, (e)Symmlet10, (f)Coiflet2, (g)Coiflet3, (h)Coiflet4, (i)Coiflet5. 

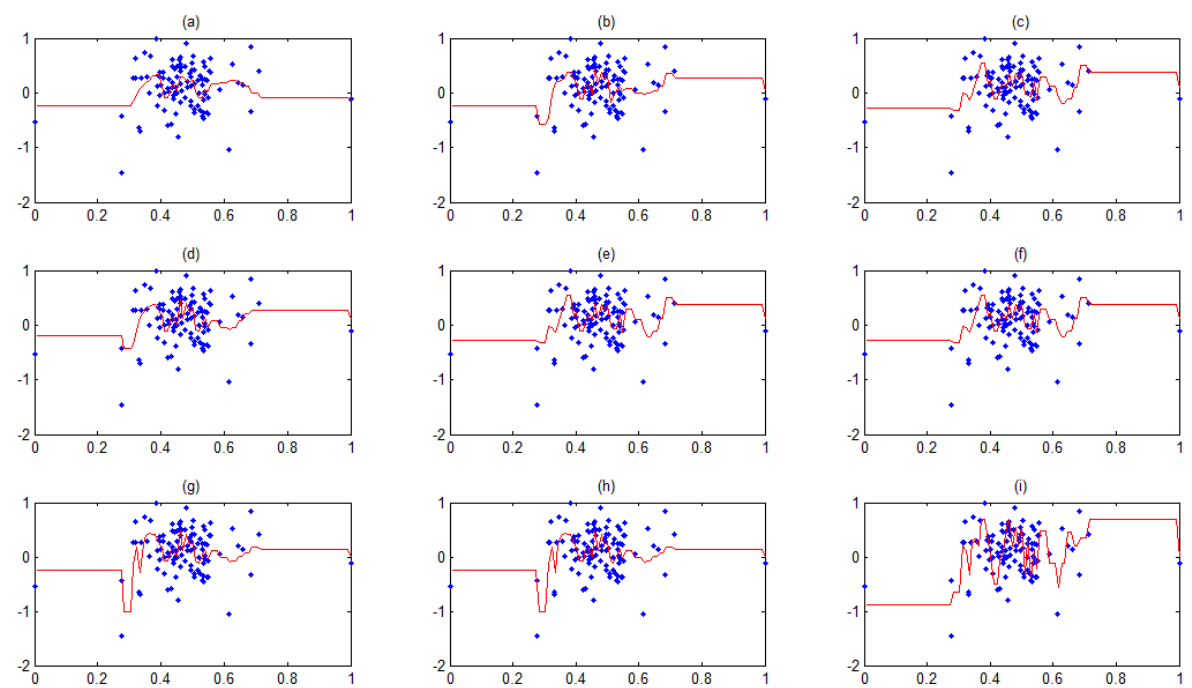

Figura A.11: Gráfico estimativas ondaleta deformada com $\mathcal{K}=1,2$ e base (a)Symmlet6, (b)Symmlet7, (c)Symmlet8, (d)Symmlet9, (e)Symmlet10, (f)Coiflet2, (g)Coiflet3, (h)Coiflet4, (i)Coiflet5.
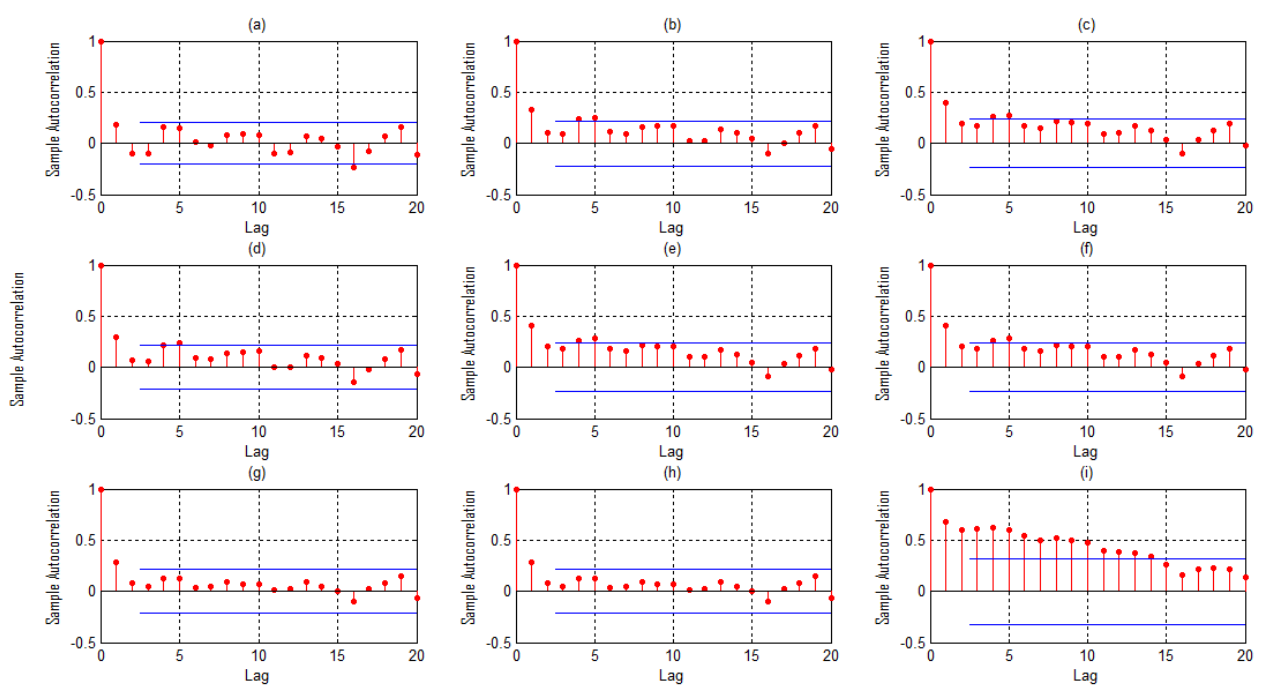

Figura A.12: Gráficos da função de autocorrelação dos resíduos das estimativas obtidas com ondaletas deformadas para $\mathcal{K}=1,2$ e base (a)Symmlet6, (b)Symmlet7, (c)Symmlet8, (d)Symmlet9, (e)Symmlet10, (f)Coiflet2, (g)Coiflet3, (h)Coiflet4, (i)Coiflet5. 


\section{Referências Bibliográficas}

Antoniadis, A. and Fan., J. (2001). Regularization of wavelet approximations. Journal of the American Statistical Association 96, 939-967. xv

Antoniadis, A., Grégoire, G. and Vial, P. (1997, October). Random design wavelet curve smoothing. Statistics $\&$ Probability Letters 35(3), 225-232. xv

Cai, T. T. and Brown, L. (1998). Wavelet shrinkage for nonequispaced sample. The Annals of Statistics 26(5), 1783-1799. 16

Chesneau, C. and Willer, T. (2007). Numerical performances of a warped wavelets estimation procedure for regression in random design. Technical report, Laboratoire de Probabilités et Modéles Aléatoires, Universités Paris. 25, 26

Dedecker, J., Doukhan, P., Lang, G., Leon, R. J., Louhichi, Sana and Prieur, C. (2007). Weak Dependence: with Examples and Applications, Volume 190 of Lecture Notes in Statistical. New York: Springer. 15

Delouille, V. (2001). Nonparametric stochastic regression with design-adapted wavelets. The Indian Journal of Statistics, Serie A 63(3), 328-366. 12, 46, 48, 53, 57, 58, 60, 61, 66

Delouille, V. (2002). Nonparametric Stochastic Regression Using Design-adapted Wavelet. Ph. D. thesis, Universite Catholique de Louvain, Paris. xv, 47, 57, 58

Delouille, V. and Sachs, V. (2004). Estimation of nonlinear autoregressive models using designadapted wavelets. Ann. Institute of Statistical of Mathematics 57(2), 235-253. xv, 43

Donoho, D. L. and Johnstone, I. M. (1994). Ideal spatial adaptation by wavelet shrinkage. Biometrika 90, 1200-1224. xv, 25, 46

Doukhan, P. (1994). Mixing: Properties and Examples, Volume 85 of Lecture Notes in Statistics. New York: Springe-Verlag. 14, 20, 36 
Doukhan, P. and Louhichi, S. (1999). A new weak dependence condition and applications to moment inequalities. Stochastic Processes and their Applications 84, 331-342. 15

Girardi, M. and Swelddens, W. (1997). A new class of unbalanced Haar wavelets that form an unconditional basis for $l_{p}$ on general measure space. J. Fourier Anal Appl 3(4), 457-474. 12

Hafner, C. (1998). Estimating high frequency foreign exchange rate volatility with nonparametric ARCH models. Journal of Statistical Planning and Inference 68, 247-269. xv

Hall, P. and Turlach, B. A. (1997.). Interpolation methods for nonlinear wavelet regression with irregularly spaced design. Annals of Statistics 25, 1912-1925. xv

Hardle, W., Kerkyacharian, G., Picard, D. and Tsybakov, A. (1998). Wavelets, Approximation, and Statistical Applications, Volume 129 of Lecture Notes in Statistical. New York: Springer. 3,5

Kerkyacharian, G. and Picard, D. (2000). Thresholding algorithms, maxisets and wellconcentrated bases. Sociedad de Estadística e Investigación Operativa Test 9(2), 283-344. 23

Kerkyacharian, G. and Picard, D. (2004). Regression in random design and warped wavelets. Bernoulli 10(6), 1053-1105. xv, 6, 9, 10, 11, 21, 22, 24, 28, 37

Morettin, P. (1999). Ondas e Ondaletas. São Paulo: Editorial Universidade de São Paulo. 1

Park, C. G., Vannucci, M. and Hart, J. D. (2005). Bayesian methods for wavelet series in single-index models. Journal of Computational and Graphical Statistics 14(4), 1-25. 77

Pereira, J. R. (2010). Estudo de correlações não lineares entre variações do Indice da Bolsa de Valores de São Paulo (ibovespa) e variação de preço de ações. Master's thesis, Universidade de São Paulo, Riberão Preto, Brasil. 69

Porto, R. (2008). Regressão não paramétrica com erros correlacionados via ondaletas. Ph. D. thesis, Universidade de São Paulo, Brasil. 9, 36

Rio, M. (1995). The funcional law of the iterated logarithm for stationary strongly mixing sequences. The Annals of Probability 23(3), 1188-1203. 43, 50

Stoker, T. M. (1986). Consistent estimation of scaled coefficients. Econometrica 54(6), 1461-81. 76 
Wang, X., Hu, S. and Yang, W. (2011). The Bahadur representation for sample quantiles under strongly mixing sequence. Journal of Statistical Planning and Inference 141,655-662. 57 